\title{
The Evolution of Compact Binary Star Systems
}

\author{
Konstantin A. Postnov \\ Sternberg Astronomical Institute \\ 13 Universitetskij Pr. \\ 119992 Moscow \\ Russia \\ email: kpostnov@gmail.com \\ http://www.sai.msu.ru \\ Lev R. Yungelson \\ Institute of Astronomy of Russian Academy of Sciences \\ 48 Pyatnitskaya Str. \\ 119017 Moscow \\ Russia \\ email: lry@inasan.ru \\ http://www.inasan.rssi.ru \\ Accepted on 13 November 2006 \\ Published on 19 December 2006

\section{Living Reviews in Relativity} \\ Published by the \\ Max Planck Institute for Gravitational Physics \\ (Albert Einstein Institute) \\ Am Mühlenberg 1, 14476 Golm, Germany \\ ISSN $1433-8351$
}

\begin{abstract}
We review the formation and evolution of compact binary stars consisting of white dwarfs (WDs), neutron stars (NSs), and black holes (BHs). Binary NSs and BHs are thought to be the primary astrophysical sources of gravitational waves (GWs) within the frequency band of ground-based detectors, while compact binaries of WDs are important sources of GWs at lower frequencies to be covered by space interferometers (LISA). Major uncertainties in the current understanding of properties of NSs and BHs most relevant to the GW studies are discussed, including the treatment of the natal kicks which compact stellar remnants acquire during the core collapse of massive stars and the common envelope phase of binary evolution. We discuss the coalescence rates of binary NSs and BHs and prospects for their detections, the formation and evolution of binary WDs and their observational manifestations. Special attention is given to AM CVn-stars - compact binaries in which the Roche lobe is filled by another WD or a low-mass partially degenerate helium-star, as these stars are thought to be the best LISA verification binary GW sources.
\end{abstract}

(c) Max Planck Society and the authors.

Further information on copyright is given at http://relativity.livingreviews .org/About/copyright.html 


\section{How to cite this article}

Owing to the fact that a Living Reviews article can evolve over time, we recommend to cite the article as follows:

Konstantin A. Postnov and Lev R. Yungelson,

"The Evolution of Compact Binary Star Systems",

Living Rev. Relativity, 9, (2006), 6. [Online Article]: cited [<date $>$ ],

http://www.livingreviews.org/lrr-2006-6

The date given as $<$ date $>$ then uniquely identifies the version of the article you are referring to.

\section{Article Revisions}

Living Reviews supports two different ways to keep its articles up-to-date:

Fast-track revision A fast-track revision provides the author with the opportunity to add short notices of current research results, trends and developments, or important publications to the article. A fast-track revision is refereed by the responsible subject editor. If an article has undergone a fast-track revision, a summary of changes will be listed here.

Major update A major update will include substantial changes and additions and is subject to full external refereeing. It is published with a new publication number.

For detailed documentation of an article's evolution, please refer always to the history document of the article's online version at http://www. livingreviews.org/lrr-2006-6. 


\section{Contents}

1 Introduction $\quad 5$

2 Observations of Double Neutron Stars $\quad 9$

2.1 Compact binaries with neutron stars . . . . . . . . . . . . . . . . 9

2.2 How frequent are double NS coalescences? . . . . . . . . . . . . . . . . . . 11

2.3 On the connection between GRBs and compact binary mergers . . . . . . . . . . 12

3 Basic Principles of Binary Star Evolution $\quad 14$

3.1 Keplerian binary system and radiation back reaction . . . . . . . . . . . . . . . 14

3.1 Keplerian motion . . . . . . . . . . . . . . . . . . . . . 14

3.1 .2 Gravitational radiation from a binary . . . . . . . . . . . . . . 15

3.1.3 Energy and angular momentum loss . . . . . . . . . . . . . . . 15

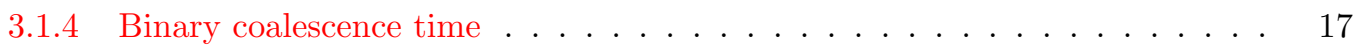

3.1.5 Magnetic stellar wind . . . . . . . . . . . . . . . . 18

3.2 Mass transfer modes and mass loss in binary systems . . . . . . . . . . . . . . . . 18

3.2 .1 Conservative accretion . . . . . . . . . . . . . . . . . . 19

3.2 .2 The Jeans (fast wind) mode . . . . . . . . . . . . . . . . . 20

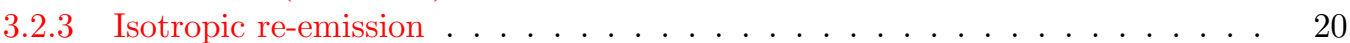

3.3 Supernova explosion . . . . . . . . . . . . . . . . . . . . . . . 21

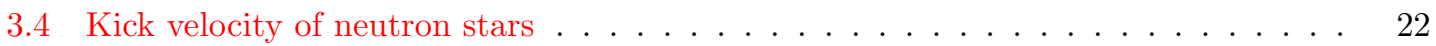

3.4.1 Effect of the kick velocity on the evolution of a binary system . . . . . . . 24

3.5 Common envelope stage . . . . . . . . . . . . . . . . . . 25

4 Evolutionary Scenario for Compact Binaries with Neutron Star or Black Hole Components

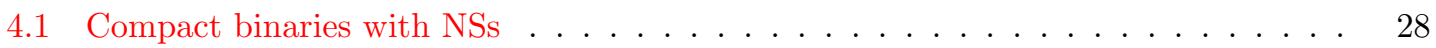

4.2 Black hole formation parameters . . . . . . . . . . . . . . . . 31

5 Formation of Double Compact Binaries $\quad 33$

5.1 Analytical estimates . . . . . . . . . . . . . . . . . . . . 33

5.2 Population synthesis results . . . . . . . . . . . . . . . . . . . 34

6 Detection Rates $\quad 36$

6.1 Enhancement of the detection rate for binary BH mergers . . . . . . . . . . . 36

6.2 Note on realistic calculation of the detection rates of binary mergings . . . . . . . . 37

6.3 Going further . . . . . . . . . . . . . . . . . . . . 37

7 Formation of Short-Period Binaries with a White-Dwarf Components 39

8 Observations $\quad \mathbf{4 7}$

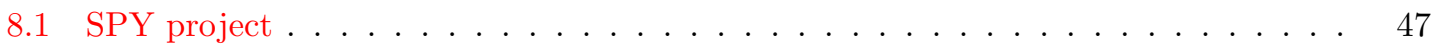

9 Evolution of Interacting Double-Degenerate Systems $\quad 49$

10 Gravitational Waves from Compact Binaries with White-Dwarf Components 52

11 AM CVn-Type Stars as Sources of Optical and X-ray Emission $\quad 59$ 
12 AM CVn-Type Stars Detectable in GWR and Electromagnetic Spectrum

12.1 Effects of finite entropy . . . . . . . . . . . . . . . . 63

12.2 Going further . . . . . . . . . . . . . . . . . . . . 65

$\begin{array}{lr}13 \text { Conclusions } & 67\end{array}$

14 Acknowledgments $\quad 68$

$\begin{array}{lr}\text { References } & 69\end{array}$ 


\section{Introduction}

Close binary stars consisting of two compact stellar remnants (white dwarfs (WDs), neutron stars (NSs), or black holes (BHs)) are considered as primary targets of the forthcoming field of gravitational wave (GW) astronomy since their orbital evolution is entirely controlled by emission of gravitational waves and leads to ultimate coalescence (merger) of the components. Close compact binaries can thus serve as testbeds for theories of gravity. The double NS(BH) mergers should be the brightest GW events in the $10-1000 \mathrm{~Hz}$ frequency band of the existing GW detectors like LIGO [16], VIRGO [5], or GEO 600 [346]. Such mergers can be accompanied by the release of a huge amount of electromagnetic energy in a burst and manifest themselves as short gamma-ray bursts (GRBs). Double WDs, especially interacting binary WDs observed as AM CVn-stars, are potential GW sources within the frequency band of the space GW interferometers like LISA [96] or future detectors [68]. The double WD mergers also stay among the primary candidate mechanisms for type Ia supernova (SN Ia) explosions, which are crucial in modern cosmological studies.

Compact binaries are the end products of the evolution of binary stars, and the main purpose of the present review is to describe the astrophysical knowledge on their formation and evolution. We shall discuss the present situation with the main parameters determining their evolution and the rates of coalescence of double NSs/BHs and WDs.

About $6 \%$ of the baryonic matter in the Universe is confined in stars [114]. The typical mass of a stationary star is close to the solar value $M_{\odot} \approx 2 \times 10^{33} \mathrm{~g}$. The minimum mass of a stationary star at the main sequence (MS) is set by the condition of stable hydrogen burning in its core $M_{\min } \approx 0.08 M_{\odot}$ [203]. The maximum mass of solar composition stars inferred observationally is close to $150 M_{\odot}$ [104]; for very low metallicity stars it is derived by the linear analysis of pulsational stability and is close to $300 M_{\odot}$ [15]. Stars and stellar systems are formed due to the development of the gravitational (Jeans) instability in turbulized molecular clouds. The minimum protostellar mass is dictated by the opacity conditions in the collapsing fragments and is found to lie in the range $0.01-0.1 M_{\odot}$ in both analytical [343] and numerical calculations (see, e.g., [74]). It is established from observations that the mass distribution of main-sequence stars has a powerlaw shape [365, 255], $d N / d M \sim M^{-\beta}$, with $\beta=-1.2$ for $0.08 \lesssim M / M_{\odot} \lesssim 0.5, \beta=-2.2$ for $0.5 \lesssim M / M_{\odot} \lesssim 1.0$, and $\beta=-2.2$ to -3.2 for $1.0 \lesssim M / M_{\odot} \lesssim 150[199,200]$.

The evolution of a single star is determined by its initial mass at the main sequence $M_{0}$ and the chemical composition. If $M_{0} \lesssim 8-12 M_{\odot}$, the carbon-oxygen $(\mathrm{CO})$ (or oxygen-neon $(\mathrm{ONe})$ at the upper end of the range) stellar core becomes degenerate and the evolution of the star ends up with the formation of a $\mathrm{CO}$ or ONe white dwarf. The formation of a WD is accompanied by the loss of stellar envelope by stellar wind in the red giant and asymptotic giant branch stages of evolution and ejection of a planetary nebula. The boundary between the masses of progenitors of WDs and NSs is not well defined and is, probably, between 8 and $12 M_{\odot}$ (cf. [161, 157, 348, 159, 115, 324, 349, 119, 377]).

At the upper boundary of the mass range of white dwarf progenitors, formation of ONe WDs is possible. The masses of stars that produce ONe WDs are still highly uncertain. However, strong observational evidence for their existence stems from the analysis of nova ejecta [405]. This variety of WDs is important in principle, because accretion induced collapse (AIC) of them may result in formation of neutron stars (see [292, 77] and references therein), but since for the purpose of detection of gravitational waves they are not different from the much more numerous CO-WDs, we will, as a rule, not consider them below as a special class.

If $M_{0} \gtrsim(10-12) M_{\odot}$, thermonuclear evolution proceeds until iron-peak elements are produced in the core. Iron cores are subjected to instabilities (neutronization, nuclei photodesintegration, or pair creation for the most massive stars) that lead to gravitational collapse. The core collapse of massive stars results in the formation of a neutron star or, for very massive stars, a black hole and is associated with the brightest astronomical phenomena such as supernova explosions 


\begin{tabular}{ccc}
\hline \hline Initial mass $\left[M_{\odot}\right]$ & remnant type & mean remnant mass $\left[M_{\odot}\right]$ \\
\hline $0.95<M<8-10$ & WD & 0.6 \\
$8-10<M<25-30$ & NS & 1.35 \\
$25-30<M<150$ & BH & $\sim 10$ \\
\hline \hline
\end{tabular}

Table 1: Types of compact stellar remnants (the ranges of progenitor mass are shown for solar composition stars).

(of type II, Ib, or Ib/c, according to the astronomical classification based on the spectra and light curves properties). If the pre-collapsing core retains significant rotation, powerful gamma-ray bursts lasting up to hundreds of seconds may be produced [456].

The boundaries between the masses of progenitors of WDs or NSs and NSs or BHs are fairly uncertain (especially for BHs). Typically accepted masses of stellar remnants for nonrotating solar chemical composition stars are summarized in Table 1.

For a more detailed introduction into the physics and evolution of stars the reader is referred to the classical textbook [67]. Formation and physics of compact objects is described in more detail in monographs [375, 35]. For a recent review of the evolution of massive stars and the mechanisms of core-collapse supernovae we refer to $[457,111,197]$.

Most stars in the Galaxy are found in multiple systems, with single stars (including our own Sun) being rather exceptions than a rule (see for example [86, 128]). In the binary stars with sufficiently large orbital separations ("wide binaries") the presence of the secondary component does not influence significantly the evolution of the components. In "close binaries" the evolutionary expansion of stars allows for a mass exchange between the components. In close binaries, the initial mass of the components at the zero-age main sequence (ZAMS) ceases to be the sole parameter determining their evolution. Consequently, the formation of compact remnants in binary stars differs from single stars. This is illustrated by Figure 1 which plots the type of the stellar remnant as a function of both initial mass and the radius of a star at the moment of the Roche-lobe overflow (RLOF). It is seen that wide binaries evolve as single stars, while for binaries with RLOF a new type of remnants appears - a helium WD, whose formation from a single star in the Hubble time is impossible ${ }^{1}$.

Binaries with compact remnants are primary potential GW sources (see Figure 2). This figure plots the sensitivity of ground-based interferometer LIGO, as well as the space laser interferometer LISA, in terms of dimensionless GW strain $h$ measured over 1 year. The strongest Galactic sources at all frequencies are the most compact double NSs and BHs. Double WDs (including AM CVnstars) and ultra-compact X-ray binaries (NS + WD) appear to be promising LISA sources.

Double NS/BH systems result from the evolution of initially massive binaries, while double WDs are formed from the evolution of low-mass binaries. We shall consider them separately.

\section{Binaries with NSs and BHs}

Binary systems with components massive enough to produce NSs or BHs at the end of thermonuclear evolution may remain bound after two supernova explosions. Then, loss of energy and momentum by GWs controls entirely their evolution and gradual reduction of the binary separation may bring the components into contact. During the merger process $10^{52} \mathrm{erg}$ are released as GWs $[58,59]$. Such strong bursts of GWs can be reliably detected by the present-day ground-based $\mathrm{GW}$ detectors from distances up to several megaparsecs and are the most important targets for GW observatories such as LIGO, GEO, and VIRGO [122, 373].

${ }^{1}$ The hydrogen burning time for single stars with $M_{0} \lesssim 0.9 M_{\odot}$ exceeds the age of the Universe.

Living Reviews in Relativity

http: //www . livingreviews . org/lrr-2006-6 


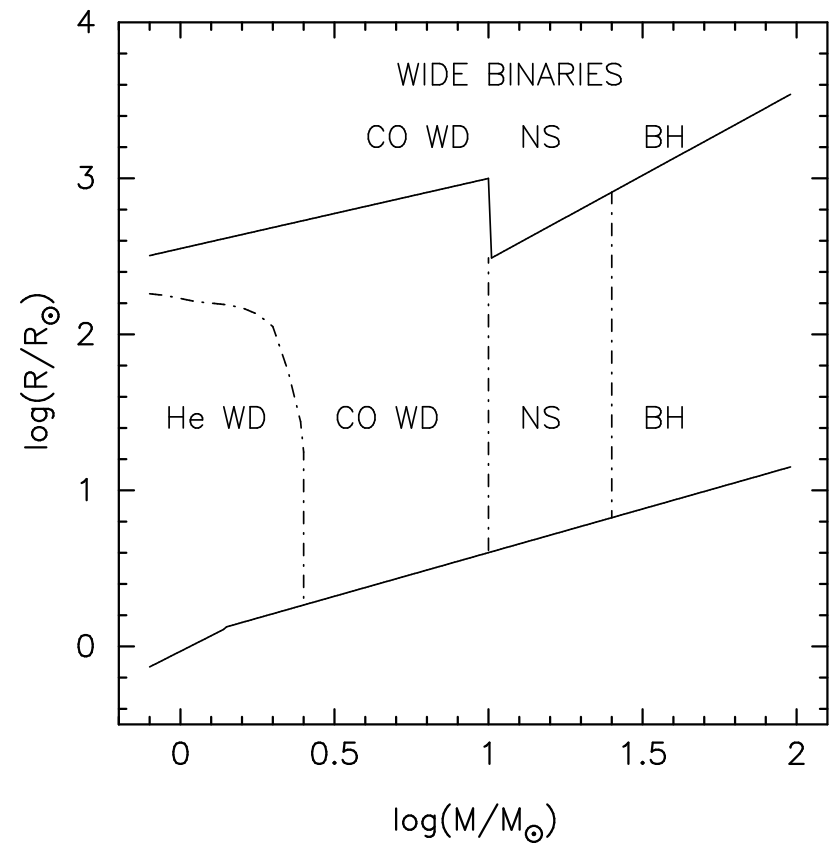

Figure 1: Descendants of components of close binaries depending on the radius of the star at $R L O F$. The boundary between progenitors of He and CO-WDs is uncertain by several $0.1 M_{\odot}$, the boundary between WDs and NSs by $\sim 1 M_{\odot}$, while for the formation of BHs the lower mass limit may be even by $\sim 10 M_{\odot}$ higher than indicated.

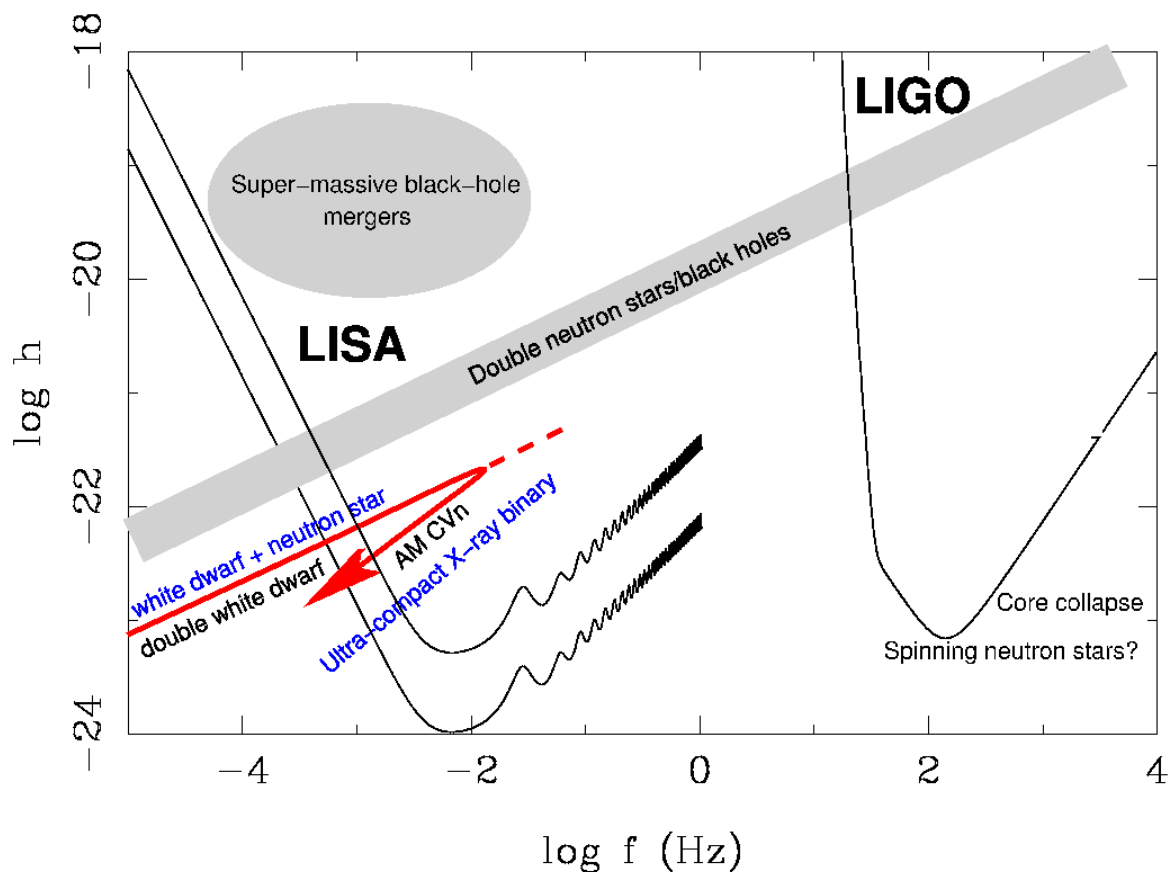

Figure 2: Sensitivity limits of $G W$ detectors and the regions of the $f-h$ diagram occupied by some of the potential $G W$ sources. (Courtesy G. Nelemans.) 
The problem is to evaluate as accurately as possible (i) the physical parameters of the coalescing binaries (masses of the components and, if possible, their spins, magnetic fields, etc.), and (ii) the occurrence rate of mergers in the Galaxy and in the local Universe. Masses of NSs in binaries are known with a rather good accuracy of $10 \%$ or better from, e.g., pulsar studies [400]; see also [211] for a recent update of NS mass measurements.

The case is not so good with the rate of coalescence of relativistic binary stars. Unfortunately, there is no way to derive it from first principles - neither the formation rate of the progenitor binaries for compact double stars nor stellar evolution are known well enough. However, the situation is not completely hopeless, especially in the case of double NS systems. Natural appearance of rotating NSs with magnetic fields as radio pulsars allows searching for binary pulsars with secondary compact companion using powerful methods of modern radio astronomy (for example, in dedicated pulsar surveys such as the Parkes multi-beam pulsar survey $[244,98])$.

Based on the observational statistics of the Galactic binary pulsars with another NS companion, one can evaluate the Galactic rate of binary NS formation and merging [313, 270, 189]. On the other hand, a direct simulation of binary star evolution in the Galaxy (the population synthesis method) can also predict the formation and merger rates of close compact binaries as a function of (numerous) parameters of binary star formation and evolution. It is important and encouraging that both estimates (observational, as inferred from recent measurements of binary pulsars [48, 180], and theoretical from the population synthesis; see Section 6) now give very close estimates for the double NS star merger rate in the Galaxy of about one event per 10,000 years. No binary BH or NS + BH systems have been found so far, so merger rates of compact binaries with $\mathrm{BHs}$ have been evaluated as yet only from population synthesis studies.

\section{Binaries with WDs}

The interest in these binaries stems from several circumstances. First, they are considered as testbeds for gravitational wave physics. Second, with them SNe Ia are associated. SNe Ia are being used as the primary standard candle sources for the determination of the cosmological parameters $\Omega$ and $\Lambda$ (see, e.g., [347, 307]). A comparison of SN Ia rates (for the different models of their progenitors) with observations may, in principle, shed light on both the star formation history and on the nature of the progenitors (see, e.g., [470, 243, 107]). The counts of distant SNe could be used to constrain cosmological parameters (see, e.g., [359]). Finally, close binaries with WDs are among the most promising verification binaries for LISA [390].

In this paper we shall concentrate on the formation and evolution of binary compact stars most relevant for GW studies. The paper is organized as follows. We start in Section 2 with a review of the main observational data on double NSs, especially measurements of masses of NSs and BHs, which are most important for the estimate of the amplitude of the expected GW signal. We briefly discuss the empirical methods to determine double NS coalescence rate. The basic principles of binary stellar evolution are discussed in Section 3. Then, in Section 4 we describe the evolution of massive binary stars. We then discuss the Galactic rate of formation of binaries with NSs and BHs in Section 5. Theoretical estimates of detection rates for mergers of binary relativistic stars are discussed in Section 6. Further we proceed to the analysis of formation of short-period binaries with WD components in Section 7, and consider observational data on binary white dwarfs in Section 8. A model for the evolution of interacting double-degenerate systems is presented in Section 9. In Section 10 we describe gravitational waves from compact binaries with white-dwarf components. Sections 11 and 12 are devoted, respectively, to the model of optical and X-ray emission of AM CVn-stars and to their subsample potentially observed both in electromagnetic and gravitational waves. Our conclusions follow in Section 13. 


\section{Observations of Double Neutron Stars}

\subsection{Compact binaries with neutron stars}

Double NSs have been discovered because one of the components of the binary is observed as a radio pulsar. The precise pulsar timing allows one to search for a periodic variation due to the binary motion. This technique is reviewed in detail by Lorimer [236]; applications of pulsar timing for general relativity tests are reviewed by Stairs [386].

Basically, pulsar timing provides the following Keplerian orbital parameters of the binary system: the binary orbital period $P_{\mathrm{b}}$ as measured from periodic Doppler variations of the pulsar spin, the projected semimajor axis $x=a \sin i$ as measured from the semi-amplitude of the pulsar radial velocity curve ( $i$ is the binary inclination angle defined such that $i=0$ for face-on systems), the orbital eccentricity $e$ as measured from the shape of the pulsar radial velocity curve, and the longitude of periastron $\omega$ at a particular epoch $T_{0}$. The first two parameters allow one to construct the mass function of the secondary companion,

$$
f\left(M_{\mathrm{p}}, M_{\mathrm{c}}\right)=\frac{4 \pi^{2} x^{3}}{P_{\mathrm{b}}^{2} T_{\odot}}=\frac{\left(M_{\mathrm{c}} \sin i\right)^{3}}{\left(M_{\mathrm{c}}+M_{\mathrm{p}}\right)^{2}}
$$

In this expression, $x$ is measured in light-seconds, $T_{\odot} \equiv G M_{\odot} / c^{3}=4.925490947 \mu \mathrm{s}$, and $M_{\mathrm{p}}$ and $M_{\mathrm{c}}$ denote masses of the pulsar and its companion, respectively. This function gives the strict lower limit on the mass of the unseen companion. However, assuming the pulsar mass to have the typical value of a NS mass (for example, confined between the lowest measured NS mass $1.25 M_{\odot}$ for PSR J0737-303B [239] and the maximum measured NS mass of $2.1 M_{\odot}$ in the NS-WD binary PSR J0751+1807 [290]), one can estimate the mass of the secondary star even without knowing the binary inclination angle $i$.

Long-term pulsar timing allows measurements of several relativistic phenomena: the advance of periastron $\dot{\omega}$, the redshift parameter $\gamma$, the Shapiro delay within the binary system qualified through post-Keplerian parameters $r, s$, and the binary orbit decay $\dot{P}_{\mathrm{b}}$. From the post-Keplerian parameters the individual masses $M_{\mathrm{p}}, M_{\mathrm{c}}$ and the binary inclination angle $i$ can be calculated [45].

Of the post-Keplerian parameters of binary pulsars, the periastron advance rate is usually measured most readily. Assuming it to be entirely due to general relativity, the total mass of the system can be evaluated:

$$
\dot{\omega}=3\left(\frac{2 \pi}{P_{\mathrm{b}}}\right)^{5 / 3} \frac{T_{\odot}^{2 / 3}\left(M_{\mathrm{c}}+M_{\mathrm{p}}\right)^{2 / 3}}{\left(1-e^{2}\right)} .
$$

High values of the derived total mass of the system $\left(\gtrsim 2.5 M_{\odot}\right)$ suggests the presence of another NS or even $\mathrm{BH}^{2}$.

If the individual masses, binary period, and eccentricity of a compact binary system are known, it is easy to calculate the time it takes for the binary companions to coalesce due to GW emission using the quadrupole formula for GW emission [308] (see Section 3.1.4 for more detail):

$$
\tau_{\mathrm{GW}} \approx 4.8 \times 10^{10} \mathrm{yr}\left(\frac{P_{\mathrm{b}}}{\mathrm{d}}\right)^{8 / 3}\left(\frac{\mu}{M_{\odot}}\right)^{-1}\left(\frac{M_{\mathrm{c}}+M_{\mathrm{p}}}{M_{\odot}}\right)^{-2 / 3}\left(1-e^{2}\right)^{7 / 2}
$$

Here $\mu=M_{\mathrm{p}} M_{\mathrm{c}} /\left(M_{\mathrm{p}}+M_{\mathrm{c}}\right)$ the reduced mass of the binary. Some observed and derived parameters of known compact binaries with NSs are collected in Tables 2 and 3.

\footnotetext{
${ }^{2}$ Unless the companion is directly observable and its mass can be estimated by other means.
} 


\begin{tabular}{l|ccccccc}
\hline \hline PSR & $\begin{array}{c}P \\
{[\mathrm{~ms}]}\end{array}$ & $\begin{array}{c}P_{\mathrm{b}} \\
{[\mathrm{d}]}\end{array}$ & $\begin{array}{c}a_{1} \sin i \\
{[\mathrm{lt}-\mathrm{s}]}\end{array}$ & $e$ & $\begin{array}{c}\dot{\omega} \\
{\left[\mathrm{deg} \mathrm{yr}^{-1}\right]}\end{array}$ & $\begin{array}{c}\dot{P}_{\mathrm{b}} \\
{\left[\times 10^{-12}\right]}\end{array}$ & Ref. \\
\hline J0737-3039A & 22.70 & 0.102 & 1.42 & 0.088 & 16.88 & -1.24 & {$[48]$} \\
$\mathrm{J} 0737-3039 \mathrm{~B}$ & 2773 & - & - & - & - & - & {$[239]$} \\
$\mathrm{J} 1518+4904$ & 40.93 & 8.634 & 20.04 & 0.249 & 0.011 & $?$ & {$[289]$} \\
$\mathrm{B} 1534+12$ & 37.90 & 0.421 & 3.73 & 0.274 & 1.756 & -0.138 & {$[455,388]$} \\
$\mathrm{J} 1756-2251$ & 28.46 & 0.320 & 2.75 & 0.181 & 2.585 & $?$ & {$[97]$} \\
$\mathrm{J} 1811-1736$ & 104.18 & 18.779 & 34.78 & 0.828 & 0.009 & $<30$ & {$[240]$} \\
$\mathrm{J} 1906+074$ & 144.07 & 0.116 & 1.42 & 0.085 & 7.57 & $?$ & {$[237]$} \\
$\mathrm{B} 1913+16$ & 59.03 & 0.323 & 2.34 & 0.617 & 4.227 & -2.428 & {$[153]$} \\
$\mathrm{B} 2127+11 \mathrm{C}$ & 30.53 & 0.335 & 2.52 & 0.681 & 4.457 & -3.937 & {$[7,332]$} \\
\hline \hline
\end{tabular}

Table 2: Observed parameters of double neutron star binaries.

\begin{tabular}{l|cccc}
\hline \hline PSR & $\begin{array}{c}f(m) \\
{\left[M_{\odot}\right]}\end{array}$ & $\begin{array}{c}M_{\mathrm{c}}+M_{\mathrm{p}} \\
{\left[M_{\odot}\right]}\end{array}$ & $\begin{array}{c}\tau_{c}=P /(2 \dot{P}) \\
{[\mathrm{Myr}]}\end{array}$ & $\begin{array}{c}\tau_{\mathrm{GW}} \\
{[\mathrm{Myr}]}\end{array}$ \\
\hline J0737-3039A & 0.29 & 2.58 & 210 & 87 \\
$\mathrm{~J} 0737-3039 \mathrm{~B}$ & - & - & 50 & - \\
$\mathrm{J} 1518+4904$ & 0.12 & 2.62 & & $9.6 \times 10^{6}$ \\
B1534+12 & 0.31 & 2.75 & 248 & 2690 \\
$\mathrm{~J} 1756-2251$ & 0.22 & 2.57 & 444 & 1690 \\
$\mathrm{~J} 1811-1736$ & 0.13 & 2.6 & & $1.7 \times 10^{6}$ \\
$\mathrm{~J} 1906+074$ & 0.11 & 2.61 & 0.112 & 300 \\
B1913+16 & 0.13 & 2.83 & 108 & 310 \\
B2127+11C & 0.15 & 2.71 & 969 & 220 \\
\hline \hline
\end{tabular}

Table 3: Derived parameters of double neutron star binaries 


\subsection{How frequent are double NS coalescences?}

As it is seen from Table 3, only six double NS systems presently known will coalesce over a time interval shorter than $\approx 10$ Gyr: J0737-3039A, B1534+12, J1756-2251, J1906+074, B1913+16, and B2127+11C. Of these six systems, one (PSR B2127+11C) is located in the globular cluster M15. This system may have a different formation history, so usually it is not included in the analysis of the coalescence rate of Galactic double compact binaries. The formation and evolution of relativistic binaries in dense stellar systems is reviewed elsewhere [26]. For a recent general review of pulsars in globular clusters see also [51].

The ordinary way of estimating the double NS merger rate from binary pulsar statistics is based on the following extrapolation [270,313]. Suppose we observe $i$ classes of Galactic binary pulsars. Taking into account various selection effects of pulsar surveys (see, e.g., [269, 189]), the Galactic number of pulsars $N_{i}$ in each class can be evaluated. To compute the Galactic merger rate of double NS binaries, we need to know the time since the birth of the NS observed as a pulsar in the given binary system. This time is the sum of the observed characteristic pulsar age $\tau_{\mathrm{c}}$ and the time required for the binary system to merge due to GW orbit decay $\tau_{\mathrm{GW}}$. With the exception of PSR J0737-3039B and the recently discovered PSR J1906+074, pulsars that we observe in binary NS systems are old recycled pulsars which were spun-up by accretion from the secondary companion to the period of several tens of ms (see Table 2). Thus their characteristic ages can be estimated as the time since termination of spin-up by accretion (for the younger pulsar PSR J0737-3039B this time can be also computed as the dynamical age of the pulsar, $P /(2 \dot{P})$, which gives essentially the same result).

Then the merger rate $\mathcal{R}_{i}$ can be calculated as $\mathcal{R}_{i} \sim N_{i} /\left(\tau_{\mathrm{c}}+\tau_{\mathrm{GW}}\right.$ ) (summed over all binary pulsars). The detailed analysis [189] indicates that the Galactic merger rate of double NSs is mostly determined by pulsars with faint radio luminosity and short orbital periods. Presently, it is the nearby $(600 \mathrm{pc})$ double-pulsar system PSR J0737-3039 with a short orbital period of $2.4 \mathrm{hr}$ [48] that mostly determines the empirical estimate of the merger rate. According to Kim et al. [190], "the most likely values of DNS merger rate lie in the range 3-190 per Myr depending on different pulsar models". The estimates by population synthesis codes are still plagued by uncertainties in statistics of binaries, in modeling binary evolution and supernovae. The most optimistic "theoretical" predictions amount to $\simeq 300 \mathrm{Myr}^{-1}[420,22]$.

Recently, the bursting radio source GCRT J1745-3009 in the direction to the Galactic centre was proposed to be a possible double NS binary [407]. The source was found to emit a series of radio bursts with high brightness temperature, of typical duration $\sim 10 \mathrm{~min}$, with an apparent periodic pattern of $\sim 77 \mathrm{~min}$ [156]. Confirmation of the binary NS nature of transient radio sources like GCRT J1745-3009 would be important to get a more precise estimate of the Galactic coalescence rate of double NS.

The extrapolation beyond the Galaxy is usually done by scaling the Galactic merger rate to the volume from which the merger events can be detected with given GW detector's sensitivity. The scaling factor widely used is the ratio between the B-band luminosity density in the local Universe, correlating with the star-formation rate, and the B-band luminosity of the Galaxy [313, 182]. One can also use for this purpose the direct ratio of the Galactic star formation rate $S_{F} R_{G} \simeq$ $3 M_{\odot} \mathrm{yr}^{-1}[255,401]$ to the star formation rate on the local Universe $\mathrm{SFR}_{\mathrm{loc}} \simeq 0.03 M_{\odot} \mathrm{yr}^{-1}[306$, 371]. These estimates yield the relation

$$
\mathcal{R}_{\mathrm{V}} \approx 0.01 \mathcal{R}_{\mathrm{G}}\left[\mathrm{Mpc}^{-3}\right] .
$$

So for the Galactic merger rate $\mathcal{R}_{\mathrm{G}} \sim 10^{-4} \mathrm{yr}^{-1}$ a very optimistic detection rate for binary NSs of about once per 1-2 years of observations by the first-generation GW detectors is predicted [48]. This estimate is still uncertain, mostly due to poor knowledge of the luminosity function for faint radio pulsars [190]. 
Recently, the first results of the search for GWs from coalescing binary systems in the Milky Way and the Magellanic Clouds using data taken by two of the three LIGO interferometers [3] established an observational upper limit to the Galactic binary NS coalescence rate of $\mathcal{R}_{\mathrm{G}}<1.7 \times 10^{2} \mathrm{yr}^{-1}$. With increasing sensitivity of GW detectors, this limit will be much improved in the nearest future.

\subsection{On the connection between GRBs and compact binary mergers}

Here we wish to mention the possibility of observational manifestations of NS/BH binary mergers other than the violent GW emission. First of all, it is the connection of relativistic binary mergers to some subclasses of cosmic GRBs. That catastrophic events like the coalescence of binary NSs or BHs can be related to GRBs has been suggested for quite a long time. First ideas can be found in the papers of Sergei Blinnikov and his coauthors [39] and Bohdan Paczyński [302] with subsequent studies in [90, 303, 258, 188], etc.

Now these ideas gained strong observational support from the accurate localization of short GRBs with hard spectrum by the Swift and HETE-II space missions [116, 40, 31, 109]. The shorthard subclass of GRBs includes up to $30 \%$ of all GRBs [299]. The most important recent discovery is that these GRBs occur both in late-type [66, 146] and early-type galaxies [31, 18], suggesting old stellar population progenitors. This is in sharp contrast to long GRBs, some of which are definitely associated with peculiar type $\mathrm{Ib} / \mathrm{c}$ supernovae produced by the core collapse of massive stars [145, 389].

The principal observational facts about several well-localized short GRBs (see [29] for more discussion) are:

1. They occur at cosmological redshifts from 0.160 to 1.8 and may constitute up to $20 \%$ of the local short GRB population detected by the $\mathrm{BATSE}^{3}$ experiment on board of the Compton Gamma-ray Observatory.

2. The isotropic energy release is typically lower than in long GRBs (from $\sim 10^{48}$ erg to $\sim$ $\left.4 \times 10^{51} \mathrm{erg}\right)$.

3. The opening angle of the ejecta in these GRBs is on average larger than in long GRBs [50].

4. Short GRBs are found both in elliptical and star forming galaxies. Statistical analysis suggests that the occurrence rate of short bursts is roughly equal in early-type and late-type galaxies [29].

The short GRB rate inferred from these observations [317], $\mathcal{R}_{\mathrm{sGRB}} \sim(10-30)_{70}^{3} \mathrm{Gpc}^{-3} \mathrm{yr}^{-1}$ agrees with the double NS merger rate derived from binary pulsar statistics. Depending on the unknown beaming factor a possible upper limit of about $10^{5}$ events $\mathrm{yr}^{-1} \mathrm{Gpc}^{-3}$ was obtained in [264]. That paper also extensively discusses the application of the rate of short GRBs to LIGO/VIRGO detections of double NS binary mergings if they are associated with short GRBs, and gives very good prospects for the Advanced LIGO sensitivity (up to hundreds detections per year). However, recent deep optical observations of several short GRBs provide evidence for their association with very faint galaxies [30], suggesting the intrinsic luminosity of a significant part of short GRBs to be much higher than $10^{48}-10^{49} \mathrm{erg}$, as inferred from observations of close short GRBs by [264], close to that of classical long GRBs $\sim 10^{51}-10^{52}$ erg. Taking this finding into account decreases the expected detection rate of NS mergers (if they are associated with short GRBs) down to several events per year by the Advanced LIGO detector [30].

We also emphasize the agreement of the observational estimates with population synthesis calculations of binary mergers in galaxies of different types [14, 305, 21, 46, 24]. The analysis of

${ }^{3}$ Burst And Transient Source Experiment [271]. 
luminosity function and statistics of short GRBs from the BATSE catalog [317] implies a delay relative to the star formation history, which can favour double NS systems dynamically formed in stellar clusters as progenitors [150,121]. Theoretical issues related to the generation of short hard GRBs from binary NS and NS-BH mergers are discussed in [212, 213, 295].

Now let us see what theory says about the formation, evolution, and detection rates of close compact binaries and their properties. 


\section{Basic Principles of Binary Star Evolution}

Beautiful general reviews of the topic can be found in [33, 425]. Here we restrict ourselves to recalling several facts about binary evolution which are most relevant to the formation and evolution of compact binaries. The readers who have experience in the field can skip this section.

\subsection{Keplerian binary system and radiation back reaction}

We start with some basic facts about Keplerian motion in a binary system and the simplest case of evolution of two point masses due to gravitational radiation losses. The stars are highly condensed objects, so their treatment as point masses is usually adequate for the description of their interaction in the binary. Furthermore, Newtonian gravitation theory is sufficient for this purpose as long as the orbital velocities are small in comparison with the speed of light $c$. The systematic change of the orbit caused by the emission of gravitational waves will be considered in a separate paragraph below.

\subsubsection{Keplerian motion}

Let us consider two point masses $M_{1}$ and $M_{2}$ orbiting each other under the force of gravity. It is well known (see [208]) that this problem is equivalent to the problem of a single body with mass $\mu$ moving in an external gravitational potential. The value of the external potential is determined by the total mass of the system

$$
M=M_{1}+M_{2} \text {. }
$$

The reduced mass $\mu$ is

$$
\mu=\frac{M_{1} M_{2}}{M} .
$$

The body $\mu$ moves in an elliptic orbit with eccentricity $e$ and major semi-axis $a$. The orbital period $P$ and orbital frequency $\Omega=2 \pi / P$ are related to $M$ and $a$ by Kepler's third law

$$
\Omega^{2}=\left(\frac{2 \pi}{P}\right)^{2}=\frac{G M}{a^{3}} .
$$

This relationship is valid for any eccentricity $e$.

Individual bodies $M_{1}$ and $M_{2}$ move around the barycentre of the system in elliptic orbits with the same eccentricity $e$. The major semi-axes $a_{i}$ of the two ellipses are inversely proportional to the masses

$$
\frac{a_{1}}{a_{2}}=\frac{M_{2}}{M_{1}}
$$

and satisfy the relationship $a=a_{1}+a_{2}$. The position vectors of the bodies from the system's barycentre are $\vec{r}_{1}=M_{2} \vec{r} /\left(M_{1}+M_{2}\right)$ and $\vec{r}_{2}=-M_{1} \vec{r} /\left(M_{1}+M_{2}\right)$, where $\vec{r}=\vec{r}_{1}-\vec{r}_{2}$ is the relative position vector. Therefore, the velocities of the bodies with respect to the system's barycentre are related by

$$
-\frac{\vec{V}_{1}}{\vec{V}_{2}}=\frac{M_{2}}{M_{1}},
$$

and the relative velocity is $\vec{V}=\vec{V}_{1}-\vec{V}_{2}$.

The total conserved energy of the binary system is

$$
E=\frac{M_{1} \vec{V}_{1}^{2}}{2}+\frac{M_{2} \vec{V}_{2}^{2}}{2}-\frac{G M_{1} M_{2}}{r}=\frac{\mu \vec{V}^{2}}{2}-\frac{G M_{1} M_{2}}{r}=-\frac{G M_{1} M_{2}}{2 a},
$$

Living Reviews in Relativity

http://www. livingreviews.org/lrr-2006-6 
where $r$ is the distance between the bodies. The orbital angular momentum vector is perpendicular to the orbital plane and can be written as

$$
\vec{J}_{\text {orb }}=M_{1} \vec{V}_{1} \times \vec{r}_{1}+M_{2} \vec{V}_{2} \times \vec{r}_{2}=\mu \vec{V} \times \vec{r} .
$$

The absolute value of the orbital angular momentum is

$$
\left|\vec{J}_{\text {orb }}\right|=\mu \sqrt{G M a\left(1-e^{2}\right)} .
$$

For circular binaries with $e=0$ the distance between orbiting bodies does not depend on time,

$$
r(t, e=0)=a,
$$

and is usually referred to as orbital separation. In this case, the velocities of the bodies, as well as their relative velocity, are also time-independent,

$$
V \equiv|\vec{V}|=\Omega a=\sqrt{G M / a},
$$

and the orbital angular momentum becomes

$$
\left|\vec{J}_{\text {orb }}\right|=\mu V a=\mu \Omega a^{2} .
$$

\subsubsection{Gravitational radiation from a binary}

The plane of the orbit is determined by the orbital angular momentum vector $\vec{J}_{\text {orb }}$. The line of sight is defined by a unit vector $\vec{n}$. The binary inclination angle $i$ is defined by the relation $\cos i=\left(\vec{n}, \vec{J}_{\text {orb }} / J_{\text {orb }}\right)$ such that $i=90^{\circ}$ corresponds to a system visible edge-on.

Let us start from two point masses $M_{1}$ and $M_{2}$ in a circular orbit. In the quadrupole approximation [209], the two polarization amplitudes of GWs at a distance $r$ from the source are given by

$$
\begin{aligned}
& h_{+}=\frac{G^{5 / 3}}{c^{4}} \frac{1}{r} 2\left(1+\cos ^{2} i\right)(\pi f M)^{2 / 3} \mu \cos (2 \pi f t), \\
& h_{\times}= \pm \frac{G^{5 / 3}}{c^{4}} \frac{1}{r} 4 \cos i(\pi f M)^{2 / 3} \mu \sin (2 \pi f t) .
\end{aligned}
$$

Here $f=\Omega / \pi$ is the frequency of the emitted GWs (twice the orbital frequency). Note that for a fixed distance $r$ and a given frequency $f$, the GW amplitudes are fully determined by $\mu M^{2 / 3}=$ $\mathcal{M}^{5 / 3}$, where the combination

$$
\mathcal{M} \equiv \mu^{3 / 5} M^{2 / 5}
$$

is called the "chirp mass" of the binary. After averaging over the orbital period (so that the squares of periodic functions are replaced by $1 / 2$ ) and the orientations of the binary orbital plane, one arrives at the averaged (characteristic) GW amplitude

$$
h(f, \mathcal{M}, r)=\left(\left\langle h_{+}^{2}\right\rangle+\left\langle h_{\times}^{2}\right\rangle\right)^{1 / 2}=\left(\frac{32}{5}\right)^{1 / 2} \frac{G^{5 / 3}}{c^{4}} \frac{\mathcal{M}^{5 / 3}}{r}(\pi f)^{2 / 3} .
$$

\subsubsection{Energy and angular momentum loss}

In the approximation and under the choice of coordinates that we are working with, it is sufficient to use the Landau-Lifshitz gravitational pseudo-tensor [209] when calculating the gravitational waves energy and flux. (This calculation can be justified with the help of a fully satisfactory gravitational energy-momentum tensor that can be derived in the field theory formulation of general 
relativity [11]). The energy $d E$ carried by a gravitational wave along its direction of propagation per area $d A$ per time $d t$ is given by

$$
\frac{d E}{d A d t} \equiv F=\frac{c^{3}}{16 \pi G}\left[\left(\frac{\partial h_{+}}{\partial t}\right)^{2}+\left(\frac{\partial h_{\times}}{\partial t}\right)^{2}\right] .
$$

The energy output $d E / d t$ from a localized source in all directions is given by the integral

$$
\frac{d E}{d t}=\int F(\theta, \phi) r^{2} d \Omega
$$

Replacing

$$
\left(\frac{\partial h_{+}}{\partial t}\right)^{2}+\left(\frac{\partial h_{\times}}{\partial t}\right)^{2}=4 \pi^{2} f^{2} h^{2}(\theta, \phi)
$$

and introducing

$$
h^{2}=\frac{1}{4 \pi} \int h^{2}(\theta, \phi) d \Omega
$$

we write Equation (19) in the form

$$
\frac{d E}{d t}=\frac{c^{3}}{G}(\pi f)^{2} h^{2} r^{2}
$$

Specifically for a binary system in a circular orbit, one finds the energy loss from the system (sign minus) with the help of Equations (20) and (17):

$$
\frac{d E}{d t}=-\left(\frac{32}{5}\right) \frac{G^{7 / 3}}{c^{5}}(\mathcal{M} \pi f)^{10 / 3}
$$

This expression is exactly the same one that can be obtained directly from the quadrupole formula [209],

$$
\frac{d E}{d t}=-\frac{32}{5} \frac{G^{4}}{c^{5}} \frac{M_{1}^{2} M_{2}^{2} M}{a^{5}}
$$

rewritten using the definition of the chirp mass and Kepler's law. Since energy and angular momentum are continuously carried away by gravitational radiation, the two masses in orbit spiral towards each other, thus increasing their orbital frequency $\Omega$. The GW frequency $f=\Omega / \pi$ and the GW amplitude $h$ are also increasing functions of time. The rate of the frequency change is ${ }^{4}$

$$
\dot{f}=\left(\frac{96}{5}\right) \frac{G^{5 / 3}}{c^{5}} \pi^{8 / 3} \mathcal{M}^{5 / 3} f^{11 / 3} .
$$

In spectral representation, the flux of energy per unit area per unit frequency interval is given by the right-hand-side of the expression

$$
\frac{d E}{d A d f}=\frac{c^{3}}{G} \frac{\pi f^{2}}{2}\left(\left|\tilde{h}(f)_{+}\right|^{2}+\left|\tilde{h}(f)_{\times}\right|^{2}\right) \equiv \frac{c^{3}}{G} \frac{\pi f^{2}}{2} S_{h}^{2}(f),
$$

where we have introduced the spectral density $S_{h}^{2}(f)$ of the gravitational wave field $h$. In the case of a binary system, the quantity $S_{h}$ is calculable from Equations $(15,16)$ :

$$
S_{h}=\frac{G^{5 / 3}}{c^{3}} \frac{\pi}{12} \frac{\mathcal{M}^{5 / 3}}{r^{2}} \frac{1}{(\pi f)^{7 / 3}} .
$$

\footnotetext{
${ }^{4} \mathrm{~A}$ signal with such an increasing frequency is reminiscent of the chirp of a bird. This explains the origin of the term "chirp mass" for the parameter $\mathcal{M}$ which fully determines the GW frequency and amplitude behaviour.
}

Living Reviews in Relativity

http: //www. livingreviews.org/lrr-2006-6 


\subsubsection{Binary coalescence time}

A binary system in a circular orbit loses energy according to Equation (21). For orbits with non-zero eccentricity $e$, the right-hand-side of this formula should be multiplied by the factor

$$
f(e)=\left(1+\frac{73}{24} e^{2}+\frac{37}{96} e^{4}\right)\left(1-e^{2}\right)^{-7 / 2}
$$

(see [308]). The initial binary separation $a_{0}$ decreases and, assuming Equation (22) is always valid, it should vanish in a time

$$
t_{0}=\frac{c^{5}}{G^{3}} \frac{5 a_{0}^{4}}{256 M^{2} \mu}=\frac{5 c^{5}}{256} \frac{\left(P_{0} / 2 \pi\right)^{8 / 3}}{(G \mathcal{M})^{5 / 3}} \approx\left(9.8 \times 10^{6} \mathrm{yr}\right)\left(\frac{P_{0}}{1 \mathrm{~h}}\right)^{8 / 3}\left(\frac{\mathcal{M}}{M_{\odot}}\right)^{-5 / 3}
$$

As we noted above, gravitational radiation from the binary depends on the chirp mass $\mathcal{M}$, which can also be written as $\mathcal{M} \equiv M \eta^{3 / 5}$, where $\eta$ is the dimensionless ratio $\eta=\mu / M$. Since $\eta \leq 1 / 4$, one has $\mathcal{M} \lesssim 0.435 M$. For example, for two NS with equal masses $M_{1}=M_{2}=1.4 M_{\odot}$, the chirp mass is $\mathcal{M} \approx 1.22 M_{\odot}$. This explains the choice of normalization in Equation (26).

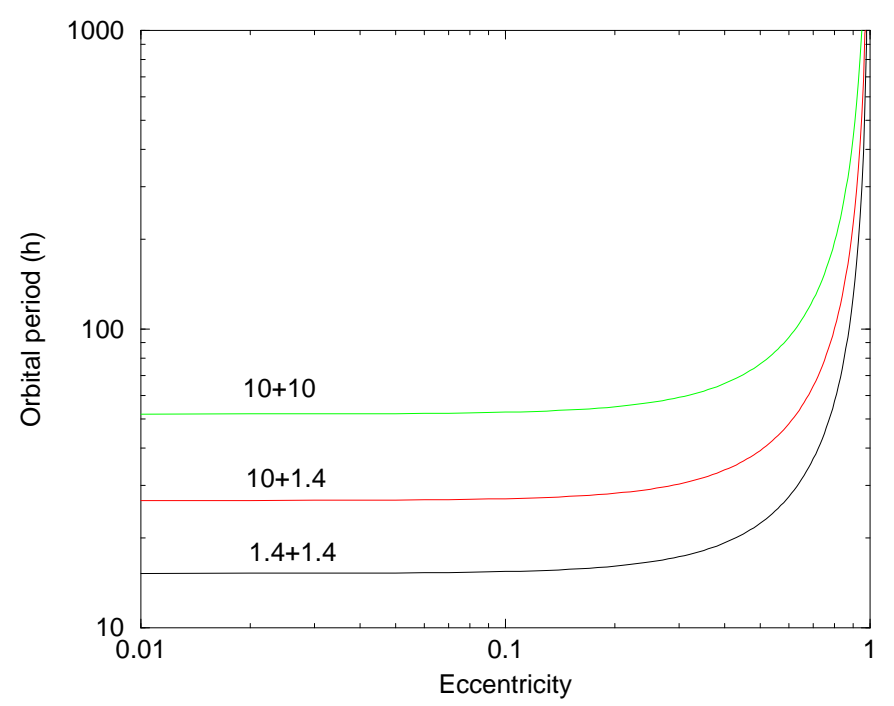

Figure 3: The maximum initial orbital period (in hours) of two point masses which will coalesce due to gravitational wave emission in a time interval shorter than $10^{10} \mathrm{yr}$, as a function of the initial eccentricity $e_{0}$. The lines are calculated for $10 M_{\odot}+10 M_{\odot}(B H+B H), 10 M_{\odot}+1.4 M_{\odot}$ $(B H+N S)$, and $1.4 M_{\odot}+1.4 M_{\odot}(N S+N S)$.

The coalescence time for an initially eccentric orbit with $e_{0} \neq 0$ and separation $a_{0}$ is shorter than the coalescence time for a circular orbit with the same initial separation $a_{0}$ [308]:

$$
t_{\mathrm{c}}\left(e_{0}\right)=t_{0} f\left(e_{0}\right),
$$

where the correction factor $f\left(e_{0}\right)$ is

$$
f\left(e_{0}\right)=\frac{48}{19} \frac{\left(1-e_{0}^{2}\right)^{4}}{e_{0}^{48 / 19}\left(1+\frac{121}{304} e_{0}^{2}\right)^{3480 / 2299}} \int_{0}^{e_{0}} \frac{\left(1+\frac{121}{304} e^{2}\right)^{1181 / 2299}}{\left(1-e^{2}\right)^{3 / 2}} e^{29 / 19} d e .
$$

To merge in a time interval shorter than $10 \mathrm{Gyr}$ the binary should have a small enough initial orbital period $P_{0} \leq P_{c r}\left(e_{0}, \mathcal{M}\right)$ and, accordingly, a small enough initial semimajor axis $a_{0} \leq$ 
$a_{c r}\left(e_{0}, \mathcal{M}\right)$. The critical orbital period is plotted as a function of the initial eccentricity $e_{0}$ in Figure 3. The lines are plotted for three typical sets of masses: two neutron stars with equal masses $\left(1.4 M_{\odot}+1.4 M_{\odot}\right)$, a black hole and a neutron star $\left(10 M_{\odot}+1.4 M_{\odot}\right)$, and two black holes with equal masses $\left(10 M_{\odot}+10 M_{\odot}\right)$. Note that in order to get a significantly shorter coalescence time, the initial binary eccentricity should be $e_{0} \geq 0.6$.

\subsubsection{Magnetic stellar wind}

In the case of low-mass binary evolution, there is another important physical mechanism responsible for the removal of orbital angular momentum, in addition to GW emission discussed above. This is the magnetic stellar wind (MSW), or magnetic braking, which is thought to be effective for main-sequence G-M dwarfs with convective envelopes, i.e. in the mass interval $0.3-1.5 M_{\odot}$. The upper mass limit corresponds to the disappearance of a deep convective zone, while the lower mass limit stands for fully convective stars. In both cases a dynamo mechanism, responsible for enhanced magnetic activity, is thought to be ineffective. The idea behind angular momentum loss (AML) by magnetically coupled stellar wind is that the stellar wind is compelled by magnetic field to corotate with the star to rather large distances where it carries away large specific angular momentum [370]. Thus, it appears possible to take away substantial angular momentum without evolutionary significant mass-loss in the wind. The concept of an MSW was introduced into analyses of the evolution of compact binaries by Verbunt and Zwaan [435] when it became evident that momentum loss by GWs alone is unable to explain the observed mass-transfer rates in cataclysmic variables. The latter authors based their reasoning on observations of the spin-down of single G-dwarfs in stellar clusters with age [378] $V \propto t^{-1 / 2}$ (the Skumanich law). Applying this to a binary component and assuming tidal locking between the star's axial rotation and orbital revolution, one arrives at the rate of angular momentum loss via an MSW,

$$
\frac{\dot{J}_{\mathrm{MSW}}}{J_{\mathrm{orb}}} \sim-\frac{R_{\mathrm{o}}^{4}}{M_{\mathrm{c}}} \frac{G M^{2}}{a^{5}},
$$

where $R_{\mathrm{O}}$ is the radius of the optical companion and $M_{\mathrm{c}}$ is the mass of the compact star.

Radii of stars filling their Roche lobes should be proportional to binary separations, $R_{\mathrm{o}} \propto a$, which means that the characteristic time of orbital angular momentum removal by an MSW is $\tau_{\mathrm{MSW}} \equiv\left(\dot{J}_{\mathrm{MSW}} / J_{\mathrm{orb}}\right)^{-1} \propto a$. This should be compared with AML by GWs with $\tau_{\mathrm{GW}} \propto a^{4}$. Clearly, the MSW (if it operates) is more effective in removing angular momentum from binary system at larger separations (orbital periods), and at small orbital periods GWs always dominate. Magnetic braking is especially important in CVs and in LMXBs with orbital periods exceeding several hours and is the driving mechanis for mass accretion onto the compact component.

We should note that the above prescription for an MSW is still debatable, since it is based on extrapolation of stellar rotation rates over several orders of magnitude - from slowly rotating field stars to rapidly spinning components of close binaries. There are strong indications that actual magnetic braking for compact binaries may be much weaker than predictions based on the Skumanich law (see, e.g., [323] for recent discussion and references).

\subsection{Mass transfer modes and mass loss in binary systems}

GW emission is the sole factor responsible for the change of orbital parameters of a pair of compact (degenerate) stars. However, at the early stages of binary evolution, it is the mass transfer between the components and the loss of matter and its orbital momentum that play a dominant dynamical role. Strictly speaking, these processes should be treated hydro-dynamically and they require complicated numerical calculations. However, binary evolution can also be described semiqualitatively, using a simplified description in terms of point-like bodies. The change of their integrated physical

Living Reviews in Relativity

http://www . livingreviews . org/lrr-2006-6 
quantities, such as masses, orbital angular momentum, etc. governs the evolution of the orbit. This description turns out to be successful in reproducing the results of more rigorous numerical calculations (see, e.g., [294] for more detail and references). In this approach, the key role is allocated to the total orbital angular momentum $J_{\text {orb }}$ of the binary.

Let star 2 lose matter at a rate $\dot{M}_{2}<0$ and let $\beta(0 \leq \beta \leq 1)$ be the fraction of the ejected matter which leaves the system (the rest falls on the first star), i.e. $\dot{M}_{1}=-(1-\beta) \dot{M}_{2} \geq 0$. Consider circular orbits with orbital angular momentum given by Equation (14). Differentiate both parts of Equation (14) by time $t$ and exclude $d \Omega / d t$ with the help of Kepler's third law (7). This gives us the rate of change of the orbital separation:

$$
\frac{\dot{a}}{a}=-2\left(1+(\beta-1) \frac{M_{2}}{M_{1}}-\frac{\beta}{2} \frac{M_{2}}{M}\right) \frac{\dot{M}_{2}}{M_{2}}+2 \frac{\dot{J}_{\text {orb }}}{J_{\text {orb }}} .
$$

In the previous equation $\dot{a}$ and $\dot{M}$ are not independent variables if the donor fills its Roche lobe. One defines the mass transfer as conservative if both $\beta=0$ and $\dot{J}_{\text {orb }}=0$. The mass transfer is called non-conservative if at least one of these conditions is violated.

It is important to distinguish some specific cases (modes) of mass transfer:

1. conservative mass transfer,

2. non-conservative Jeans mode of mass loss (or fast wind mode),

3. non-conservative isotropic re-emission,

4. sudden mass loss from one of the component during supernova explosion, and

5. common-envelope stage.

As specific cases of angular momentum loss we consider GW emission (see Section 3.1.3 and 3.1.4) and a magnetically coupled stellar wind (see Section 3.1.5) which drive the orbital evolution for short-period binaries. For non-conservative modes, one can also introduce some subcases, such as, for example, a ring-like mode in which a circumbinary ring of expelled matter is being formed (see, e.g., [380]). Here, we will not go into the details of such sub-cases.

\subsubsection{Conservative accretion}

In the case of conservative accretion, matter from $M_{2}$ is fully deposited onto $M_{1}$. The transfer process preserves the total mass $(\beta=0)$ and the orbital angular momentum of the system. It follows from Equation (30) that

$$
M_{1} M_{2} \sqrt{a}=\text { const, }
$$

so that the initial and final binary separations are related as

$$
\frac{a_{\mathrm{f}}}{a_{\mathrm{i}}}=\left(\frac{M_{1 \mathrm{i}} M_{2 \mathrm{i}}}{M_{1 \mathrm{f}} M_{2 \mathrm{f}}}\right)^{2} .
$$

The well-known "rule of thumb" for this case says that the orbit shrinks when the more massive component loses matter, and the orbit widens in the opposite situation. During such a mass exchange, the orbital separation passes through a minimum, if the masses become equal in course of mass transfer. 


\subsubsection{The Jeans (fast wind) mode}

In this mode the ejected matter completely escapes from the system, that is, $\beta=1$. The escape of matter can take place either in a spherically symmetric way or in the form of bipolar jets moving from the system at high velocity. In both cases, matter carries away some amount of the total orbital momentum proportional to the orbital angular momentum of the mass losing star $J_{2}=\left(M_{1} / M\right) J_{\text {orb }}$ (we neglect a possible proper rotation of the star, see [425]). For the loss of orbital momentum $\dot{J}_{\text {orb }}$ it is reasonable to take

$$
\dot{J}_{\text {orb }}=\frac{\dot{M}_{2}}{M_{2}} J_{2} .
$$

In the case $\beta=1$, Equation (30) can be written as

$$
\frac{\left(\Omega \dot{a}^{2}\right)}{\Omega a^{2}}=\frac{\dot{J}_{\text {orb }}}{J_{\text {orb }}}-\frac{M_{1} \dot{M}_{2}}{M M_{2}} .
$$

Then Equation (33) in conjunction with Equation (32) gives $\Omega a^{2}=$ const, that is, $\sqrt{G a M}=$ const. Thus, as a result of such a mass loss, the change in orbital separation is

$$
\frac{a_{\mathrm{f}}}{a_{\mathrm{i}}}=\frac{M_{\mathrm{i}}}{M_{\mathrm{f}}} .
$$

Since the total mass decreases, the orbit always widens.

\subsubsection{Isotropic re-emission}

The matter lost by star 2 can first accrete to star 1 , and then, a fraction $\beta$ of the accreted matter, can be expelled from the system. This happens, for instance, when a massive star transfers matter to a compact star on the thermal timescale $\left(<10^{6}\right.$ years $)$. The accretion luminosity may exceed the Eddington luminosity limit, and the radiation pressure pushes the infalling matter away from the system, in a manner similar to the spectacular example of the SS 433 binary system. Another examples may be systems with helium stars transferring mass onto relativistic objects [234, 117] or hot white dwarf components of cataclysmic variables losing mass by optically thick winds [187]. The same algorithm may be applied to the time-averaged mass loss from novae [466]. In this mode of mass-transfer, the binary orbital momentum carried away by the expelled matter is determined by the orbital momentum of the accreting star $M_{1}$, rather than by the orbital momentum of the mass-losing star $M_{2}$. The orbital momentum loss can be written as

$$
\dot{J}_{\text {orb }}=\beta \frac{\dot{M}_{2}}{M_{1}} J_{1},
$$

where $J_{1}=\left(M_{2} / M\right) J_{\text {orb }}$ is the orbital momentum of the star $M_{1}$. In the limiting case when all the mass attracted by $M_{1}$ is expelled from the system, $\beta=1$, Equation (35) simplifies to

$$
\frac{\dot{J}_{\text {orb }}}{J_{\text {orb }}}=\frac{\dot{M}_{2} M_{2}}{M_{1} M} .
$$

After substitution of this formula into Equation (30) and integration over time, one arrives at

$$
\frac{a_{\mathrm{f}}}{a_{\mathrm{i}}}=\frac{M_{\mathrm{i}}}{M_{\mathrm{f}}}\left(\frac{M_{2 \mathrm{i}}}{M_{2 \mathrm{f}}}\right)^{2} \exp \left(-2 \frac{M_{2 \mathrm{i}}-M_{2 \mathrm{f}}}{M_{1}}\right) .
$$

The exponential term makes this mode of the mass transfer very sensitive to the components mass ratio. If $M_{1} / M_{2} \ll 1$, the separation $a$ between the stars may decrease so much that the approximation of point masses becomes invalid. The tidal orbital instability (Darwin instability) may set in, and the compact star may start spiraling toward the companion star center (the common envelope stage; see Section 3.5 below).

Living Reviews in Relativity

http://www . livingreviews . org/Irr-2006-6 


\subsection{Supernova explosion}

A supernova explosion in a massive binary system occurs on a timescale much shorter than the orbital period, so the loss of mass is practically instantaneous. This case can be treated analytically (see, e.g., [38, 106, 257, 392, 140, 460, 42, 178, 397]).

In general, the loss of matter and radiation is non-spherical, so that the remnant of the supernova explosion (neutron star or black hole) acquires some recoil velocity called kick velocity $\vec{w}$. In a binary, the kick velocity should be added to the orbital velocity of the pre-supernova star.

The usual treatment proceeds as follows. Let us consider a pre-SN binary with initial masses $M_{1}$ and $M_{2}$. The stars move in a circular orbit with orbital separation $a_{\mathrm{i}}$ and relative velocity $\vec{V}_{\mathrm{i}}$. The star $M_{1}$ explodes leaving a compact remnant of mass $M_{\mathrm{c}}$. The total mass of the binary decreases by the amount $\Delta M=M_{1}-M_{\mathrm{c}}$. It is usually assumed that the compact star acquires some additional velocity (kick velocity) $\vec{w}$ (see detailed discussion in Section 3.4). Unless the binary is disrupted, it will end up in a new orbit with eccentricity $e$, major semi axis $a_{\mathrm{f}}$, and angle $\theta$ between the orbital planes before and after the explosion. In general, the new barycentre will also receive some velocity, but we neglect this motion. The goal is to evaluate the parameters $a_{\mathrm{f}}$, $e$, and $\theta$.

It is convenient to work in an instantaneous reference frame centered on $M_{2}$ right at the time of explosion. The $x$-axis is the line from $M_{2}$ to $M_{1}$, the $y$-axis points in the direction of $\vec{V}_{\mathrm{i}}$, and the $z$-axis is perpendicular to the orbital plane. In this frame, the pre-SN relative velocity is $\vec{V}_{\mathrm{i}}=\left(0, V_{\mathrm{i}}, 0\right)$, where $V_{\mathrm{i}}=\sqrt{G\left(M_{1}+M_{2}\right) / a_{\mathrm{i}}}$ (see Equation (13)). The initial total orbital momentum is $\overrightarrow{J_{i}}=\mu_{\mathrm{i}} a_{\mathrm{i}}\left(0,0,-V_{\mathrm{i}}\right)$. The explosion is considered to be instantaneous. Right after the explosion, the position vector of the exploded star $M_{1}$ has not changed: $\vec{r}=\left(a_{\mathrm{i}}, 0,0\right)$. However, other quantities have changed: $\vec{V}_{\mathrm{f}}=\left(w_{x}, V_{\mathrm{i}}+w_{y}, w_{z}\right)$ and $\vec{J}_{\mathrm{f}}=\mu_{\mathrm{f}} a_{\mathrm{i}}\left(0, w_{z},-\left(V_{\mathrm{i}}+w_{y}\right)\right)$, where $\vec{w}=\left(w_{x}, w_{y}, w_{z}\right)$ is the kick velocity and $\mu_{\mathrm{f}}=M_{\mathrm{c}} M_{2} /\left(M_{\mathrm{c}}+M_{2}\right)$ is the reduced mass of the system after explosion. The parameters $a_{\mathrm{f}}$ and $e$ are being found from equating the total energy and the absolute value of orbital momentum of the initial circular orbit to those of the resulting elliptical orbit (see Equations $(10,14,12)$ ):

$$
\begin{gathered}
\mu_{\mathrm{f}} \frac{V_{\mathrm{f}}^{2}}{2}-\frac{G M_{\mathrm{c}} M_{2}}{a_{\mathrm{i}}}=-\frac{G M_{\mathrm{c}} M_{2}}{2 a_{\mathrm{f}}}, \\
\mu_{\mathrm{f}} a_{\mathrm{i}} \sqrt{w_{z}^{2}+\left(V_{\mathrm{i}}+w_{y}\right)^{2}}=\mu_{\mathrm{f}} \sqrt{G\left(M_{\mathrm{c}}+M_{2}\right) a_{\mathrm{f}}\left(1-e^{2}\right)} .
\end{gathered}
$$

For the resulting $a_{\mathrm{f}}$ and $e$ one finds

$$
\frac{a_{\mathrm{f}}}{a_{\mathrm{i}}}=\left[2-\chi\left(\frac{w_{x}^{2}+w_{z}^{2}+\left(V_{\mathrm{i}}+w_{y}\right)^{2}}{V_{\mathrm{i}}^{2}}\right)\right]^{-1}
$$

and

$$
1-e^{2}=\chi \frac{a_{\mathrm{i}}}{a_{\mathrm{f}}}\left(\frac{w_{z}^{2}+\left(V_{\mathrm{i}}+w_{y}\right)^{2}}{V_{\mathrm{i}}^{2}}\right),
$$

where $\chi \equiv\left(M_{1}+M_{2}\right) /\left(M_{\mathrm{c}}+M_{2}\right) \geq 1$. The angle $\theta$ is defined by

$$
\cos \theta=\frac{\overrightarrow{J_{\mathrm{f}}} \cdot \overrightarrow{J_{\mathrm{i}}}}{\left|\overrightarrow{J_{\mathrm{f}}}\right|\left|\overrightarrow{J_{\mathrm{i}}}\right|}
$$

which results in

$$
\cos \theta=\frac{V_{\mathrm{i}}+w_{y}}{\sqrt{w_{z}^{2}+\left(V_{\mathrm{i}}+w_{y}\right)^{2}}} .
$$

The condition for disruption of the binary system depends on the absolute value $V_{\mathrm{f}}$ of the final velocity, and on the parameter $\chi$. The binary disrupts if its total energy defined by the 
left-hand-side of Equation (38) becomes non-negative or, equivalently, if its eccentricity defined by Equation (41) becomes $e \geq 1$. From either of these requirements one derives the condition for disruption:

$$
\frac{V_{\mathrm{f}}}{V_{\mathrm{i}}} \geq \sqrt{\frac{2}{\chi}}
$$

The system remains bound if the opposite inequality is satisfied. Equation (43) can also be written in terms of the escape (parabolic) velocity $V_{\mathrm{e}}$ defined by the requirement

$$
\mu_{\mathrm{f}} \frac{V_{\mathrm{e}}^{2}}{2}-\frac{G M_{\mathrm{c}} M_{2}}{a_{\mathrm{i}}}=0 .
$$

Since $\chi=M /(M-\Delta M)$ and $V_{\mathrm{e}}^{2}=2 G(M-\Delta M) / a_{\mathrm{i}}=2 V_{\mathrm{i}}^{2} / \chi$, one can write Equation (43) in the form

$$
V_{\mathrm{f}} \geq V_{\mathrm{e}}
$$

The condition of disruption simplifies in the case of a spherically symmetric SN explosion, that is, when there is no kick velocity, $\vec{w}=0$, and, therefore, $V_{\mathrm{f}}=V_{\mathrm{i}}$. In this case, Equation (43) reads $\chi \geq 2$, which is equivalent to $\Delta M \geq M / 2$. Thus, the system unbinds if more than a half of the mass of the binary is lost. In other words, the resulting eccentricity

$$
e=\frac{M_{1}-M_{\mathrm{c}}}{M_{\mathrm{c}}+M_{2}}
$$

following from Equations $(40,41)$, and $\vec{w}=0$ becomes larger than 1 , if $\Delta M>M / 2$.

So far, we have considered an originally circular orbit. If the pre-SN star moves in an originally eccentric orbit, the condition of disruption of the system under symmetric explosion reads

$$
\Delta M=M_{1}-M_{\mathrm{c}}>\frac{1}{2} \frac{r}{a_{\mathrm{i}}},
$$

where $r$ is the distance between the components at the moment of explosion.

\subsection{Kick velocity of neutron stars}

The kick velocity imparted to a NS at birth is one of the major problems in the theory of stellar evolution. By itself it is an additional parameter, the introduction of which has been motivated first of all by high space velocities of radio pulsars inferred from the measurements of their proper motions and distances. Pulsars were recognized as a high-velocity Galactic population soon after their discovery in 1968 [125]. Shklovskii [376] put forward the idea that high pulsar velocities may result from asymmetric supernova explosions. Since then this hypothesis has been tested by pulsar observations, but no definite conclusions on its magnitude and direction have been obtained as yet.

Indeed, the distance to a pulsar is usually derived from the dispersion measure evaluation and crucially depends on the assumed model of the electron density distribution in the Galaxy. In the middle of the 1990s, Lyne and Lorimer [241] derived a very high mean space velocity of pulsars with known proper motion of about $450 \mathrm{~km} \mathrm{~s}^{-1}$. This value was difficult to adopt without invoking an additional natal kick velocity of NSs. It was suggested [225] that the observed 2D pulsar velocity distribution found by Lyne and Lorimer [241] is reproduced if the absolute value of the (assumed to be isotropic) NS kick has a power-law shape,

$$
f_{\mathrm{LL}}(|\vec{w}|) \propto \frac{\left(|\vec{w}| / w_{0}\right)^{0.19}}{\left(1+\left(|\vec{w}| / w_{0}\right)^{6.72}\right)^{0.5}}
$$

with $w_{0} \approx 400 \mathrm{~km} \mathrm{~s}^{-1}$. However, using this formula or a Maxwellian distribution for NS kicks in population synthesis calculations [225] give similar results, and later we shall not distinguish these

Living Reviews in Relativity

http: //www. livingreviews.org/lrr-2006-6 
kicks. The high mean space velocity of pulsars, consistent with earlier results by Lyne and Lorimer, was confirmed by the analysis of a larger sample of pulsars [147]. The recovered distribution of $3 \mathrm{D}$ velocities is well fit by a Maxwellian distribution with the mean value $w_{0}=400 \pm 40 \mathrm{~km} \mathrm{~s}^{-1}$ and a $1 \mathrm{D} \mathrm{rms} \sigma=265 \mathrm{~km} \mathrm{~s}^{-1}$.

Possible physical reasons for natal NS kicks due to hydrodynamic effects in core-collapse supernovae are summarized in [207, 206]. Neutrino effects in the strong magnetic field of a young NS may be also essential in explaining kicks up to $\sim 100 \mathrm{~km} \mathrm{~s}^{-1}[56,83,205]$. Astrophysical arguments favouring a kick velocity are also summarized in [398]. To get around the theoretical difficulty of insufficient rotation of pre-supernova cores in single stars to produce rapidly spinning young pulsars, Spruit and Phinney [385] proposed that random off-center kicks can lead to a net spin-up of proto-NSs. In this model, correlations between pulsar space velocity and rotation are possible and can be tested in further observations.

Here we should note that the existence of some kick follows not only from the measurements of radio pulsar space velocities, but also from the analysis of binary systems with NSs. The impact of a kick velocity $\sim 100 \mathrm{~km} \mathrm{~s}^{-1}$ explains the precessing binary pulsar orbit in PSR J00457319 [186]. The evidence of the kick velocity is seen in the inclined, with respect to the orbital plane, circumstellar disk around the Be star SS 2883 - an optical component of binary PSR B125963 [334].

Long-term pulse profile changes interpreted as geodetic precession are observed in the relativistic binary pulsars PSR 1913+16 [447], PSR B1534+12 [387], PSR J1141-6545 [151], and PSR J0737-3039B [49]. These observations indicate that in order to produce the misalignment between the orbital angular momentum and the neutron star spin, a component of the kick velocity perpendicular to the orbital plane is required $[450,453,454]$. This idea seems to gain observational support from recent thorough polarization measurements [173] suggesting alignment of the rotational axes with pulsar's space velocity. Such an alignment acquired at birth may indicate the kick velocity directed preferably along the rotation of the proto-NS. For the first SN explosion in a close binary system this would imply the kick to be perpendicular to the orbital plane.

It is worth noticing that the analysis of the formation of the double relativistic pulsar PSR J07373039 [322] may suggest, from the observed low eccentricity of the system $e \simeq 0.09$, that a small (if any) kick velocity may be acquired if the formation of the second NS in the system is associated with the collapse of an ONeMg WD due to electron-captures. The symmetric nature of electroncapture supernovae was discussed in [319] and seems to be an interesting issue requiring further studies (see, e.g., [310, 204] for the analysis of the formation of NSs in globular clusters in the frame of this hypothesis). Note that electron-capture SNe are expected to be weak events, irrespective of whether they are associated with the core-collapse of a star which retained some original envelope or with the AIC of a WD [349, 192, 77].

We also note the hypothesis of Pfahl et al. [312], based on observations of high-mass X-ray binaries with long orbital periods $(\gtrsim 30 \mathrm{~d})$ and low eccentricities $(e<0.2)$, that rapidly rotating precollapse cores may produce neutron stars with relatively small kicks, and vice versa for slowly rotating cores. Then, large kicks would be a feature of stars that retained deep convective envelopes long enough to allow a strong magnetic torque, generated by differential rotation between the core and the envelope, to spin the core down to the very slow rotation rate of the envelope. A low kick velocity imparted to the second (younger) neutron star $\left(<50 \mathrm{~km} \mathrm{~s}^{-1}\right)$ was inferred from the analysis of large-eccentricity binary pulsar PSR J1811-1736 [64]. The large orbital period of this binary pulsar (18.8 d) then may suggest an evolutionary scenario with inefficient (if any) common envelope stage [79], i.e. the absence of deep convective shell in the supernova progenitor (a He-star). This conclusion can be regarded as supportive to ideas put forward in [312].

In principle, it is possible to assume some kick velocity during $\mathrm{BH}$ formation as well [227, 112, $328,330,285,22,469]$. The similarity of NS and $\mathrm{BH}$ distribution in the Galaxy suggesting $\mathrm{BH}$ kicks was noted in [174]. A recent analysis of the space velocity of some BH binary systems [452] 
put an upper limit on the BH kick velocity of less than $\sim 200 \mathrm{~km} \mathrm{~s}^{-1}$. However, no kick seems to be required to explain the formation of other BH candidates (Cyg X-1, X-Nova Sco, etc.) [282].

To summarize, the kick velocity remains one of the important unknown parameters of binary evolution with NSs and BHs. Further constraining this parameter from various observations and theoretical understanding of possible asymmetry of core-collapse supernovae seem to be of paramount importance for the formation and evolution of close compact binaries.

\subsubsection{Effect of the kick velocity on the evolution of a binary system}

The collapse of a star to a BH, or its explosion leading to the formation of a NS, are normally considered as instantaneous. This assumption is well justified in binary systems, since typical orbital velocities before the explosion do not exceed a few hundred $\mathrm{km} / \mathrm{s}$, while most of the mass is expelled with velocities about several thousand $\mathrm{km} / \mathrm{s}$. The exploding star $M_{1}$ leaves the remnant $M_{\mathrm{c}}$, and the binary loses a portion of its mass: $\Delta M=M_{1}-M_{\mathrm{c}}$. The relative velocity of stars before the event is

$$
V_{\mathrm{i}}=\sqrt{G\left(M_{1}+M_{2}\right) / a_{\mathrm{i}}} .
$$

Right after the event, the relative velocity is

$$
\vec{V}_{\mathrm{f}}=\vec{V}_{\mathrm{i}}+\vec{w} .
$$

Depending on the direction of the kick velocity vector $\vec{w}$, the absolute value of $\vec{V}_{\mathrm{f}}$ varies in the interval from the smallest $V_{\mathrm{f}}=\left|V_{\mathrm{i}}-w\right|$ to the largest $V_{\mathrm{f}}=V_{\mathrm{i}}+w$. The system gets disrupted if $V_{\mathrm{f}}$ satisfies the condition (see Section 3.3)

$$
V_{\mathrm{f}} \geq V_{\mathrm{i}} \sqrt{\frac{2}{\chi}},
$$

where $\chi \equiv\left(M_{1}+M_{2}\right) /\left(M_{\mathrm{c}}+M_{2}\right)$.

Let us start from the limiting case when the mass loss is practically zero $(\Delta M=0, \chi=1)$, while a non-zero kick velocity can still be present. This situation can be relevant to $\mathrm{BH}$ formation. It follows from Equation (49) that, for relatively small kicks, $w<(\sqrt{2}-1) V_{\mathrm{i}}$, the system always (independently of the direction of $\vec{w})$ remains bound, while for $w>(\sqrt{2}+1) V_{\mathrm{i}}$ the system always unbinds. By averaging over equally probable orientations of $\vec{w}$ with a fixed amplitude $w$, one can show that in the particular case $w=V_{\mathrm{i}}$ the system disrupts or survives with equal probabilities. If $V_{\mathrm{f}}<V_{\mathrm{i}}$, the semimajor axis of the system becomes smaller than the original binary separation, $a_{\mathrm{f}}<a_{\mathrm{i}}$ (see Equation (40)). This means that the system becomes more hard than before, i.e. it has a greater negative total energy than the original binary. If $V_{\mathrm{i}}<V_{\mathrm{f}}<\sqrt{2} V_{\mathrm{i}}$, the system remains bound, but $a_{\mathrm{f}}>a_{\mathrm{i}}$. For small and moderate kicks $w \gtrsim V_{\mathrm{i}}$, the probabilities for the system to become more or less bound are approximately equal.

In general, the binary system loses some fraction of its mass $\Delta M$. In the absence of the kick, the system remains bound if $\Delta M<M / 2$ and gets disrupted if $\Delta M \geq M / 2$ (see Section 3.3). Clearly, a properly oriented kick velocity (directed against the vector $\vec{V}_{\mathrm{i}}$ ) can keep the system bound, even if it would have been disrupted without the kick. And, on the other hand, an unfortunate direction of $\vec{w}$ can disrupt the system, which otherwise would stay bound.

Consider, first, the case $\Delta M<M / 2$. The parameter $\chi$ varies in the interval from 1 to 2 , and the escape velocity $V_{\mathrm{e}}$ varies in the interval from $\sqrt{2} V_{\mathrm{i}}$ to $V_{\mathrm{i}}$. It follows from Equation (44) that the binary always remains bound if $w<V_{\mathrm{e}}-V_{\mathrm{i}}$, and always unbinds if $w>V_{\mathrm{e}}+V_{\mathrm{i}}$. This is a generalization of the formulae derived above for the limiting case $\Delta M=0$. Obviously, for a given $w$, the probability for the system to disrupt or become softer increases when $\Delta M$ becomes larger. Now turn to the case $\Delta M>M / 2$. The escape velocity of the compact star becomes $V_{\mathrm{e}}<V_{\mathrm{i}}$. The binary is always disrupted if the kick velocity is too large or too small: $w>V_{\mathrm{i}}+V_{\mathrm{e}}$ or $w<V_{\mathrm{i}}-V_{\mathrm{e}}$.

Living Reviews in Relativity

http: //www . livingreviews . org/lrr-2006-6 
However, for all intermediate values of $w$, the system can remain bound, and sometimes even more bound than before, if the direction of $\vec{w}$ happened to be approximately opposite to $\vec{V}_{\mathrm{i}}$. A detailed calculation of the probabilities for the binary survival or disruption requires integration over the kick velocity distribution function $f(\vec{w})$ (see, e.g., [43]).

\subsection{Common envelope stage}

This is a very important stage in the evolution of binaries of all masses. In different contexts, the evolution of binary components in an envelope engulfing both of them was considered, for instance, in [344, 384, 6], but the importance of common envelopes was really recognized after Paczyński [301] and Ostriker [298] applied them to explain the formation of cataclysmic variables and massive Xray binaries, respectively. A detailed review of problems related to common envelopes may be found, e.g., in $[158,393]$.

Generally, common envelopes form in binary systems where the mass transfer from the masslosing star is high, and the companion cannot accommodate all the accreting matter. The common envelope stage appears unavoidable on observational grounds. The evidence for a dramatic orbital angular momentum decrease in some preceding evolutionary stage follows from observations of certain types of close binary stars. They include cataclysmic variables, in which a white dwarf accretes matter from a small red dwarf main-sequence companion, planetary nebulae with double cores, low-mass X-ray binaries and X-ray transients (neutron stars and black holes accreting matter from low-mass main-sequence dwarfs). The radii of progenitors of compact stars in these binaries typically should have been 100-1000 solar radii, that is, much larger than the currently observed binary separations. This testifies of some dramatic reduction of the orbital momentum in the earlier stages of evolution and eventual removal of the common envelope. Additional indirect evidence for reality of the common envelope stage in the typical pre-cataclysmic binary V471 Tau has recently been obtained from X-ray Chandra observations [85] showing anomalous $\mathrm{C} / \mathrm{N}$ contamination of the K-dwarf companion. Recent studies also indicate that many planetary nebulae are actually binaries, which may suggest that most of them result from common envelope interaction [474, 73].

Exact criteria for the formation of a common envelope are absent. However, a high rate of mass overflow onto a compact star from a normal star is always expected when the normal star goes off the main sequence and develops a deep convective envelope. The physical reason for this is that convection tends to make entropy constant along the radius, so the radial structure of convective stellar envelopes is well described by a polytrope (i.e. the equation of state can be written as $P=K \rho^{1+1 / n}$ ) with an index $n=3 / 2$. The polytropic approximation with $n=3 / 2$ is also valid for degenerate white dwarfs with masses not too close to the Chandrasekhar limit. For a star in hydrostatic equilibrium, this results in the well known inverse mass-radius relation, $R \propto M^{-1 / 3}$, first measured for white dwarfs. Removing mass from a star with a negative power of the mass-radius relation increases its radius. On the other hand, the Roche lobe of the more massive star should shrink in response to the conservative mass exchange between the components. This further increases the mass loss rate from the Roche-lobe filling star leading to a continuation of an unstable mass loss and eventual formation of a common envelope. The critical mass ratio for the unstable Roche lobe overflow depends on specifics of the stellar structure and mass ratio of components; typically, mass loss is unstable for stars with convective envelopes, stars with radiative envelopes if $q \gtrsim 2$, and white dwarfs if $q \gtrsim 2 / 3$.

As other examples for the formation of a common envelope one may consider, for instance, direct penetration of a compact star into the dense outer layers of the companion, which can happen as a result of the Darwin tidal orbital instability in binaries [65, 12]; it is possible that a compact remnant of a supernova explosion with appropriately directed kick velocity finds itself in an elliptic orbit whose minimum periastron distance $a_{\mathrm{f}}(1-e)$ is smaller than the stellar radius of the companion; a common envelope enshrouding both components of a binary may form due to 
unstable thermonuclear burning in the surface layers of an accreting WD.

The common envelope stage is, usually, treated in the following simplified way [444, 72]. The orbital evolution of the compact star $m$ inside the envelope of the normal star $M_{1}$ is driven by the dynamical friction drag. This leads to a gradual spiral-in process of the compact star. The released orbital energy $\Delta E_{\text {orb }}$, or a fraction of it, can become numerically equal to the binding energy $E_{\text {bind }}$ of the envelope with the rest of the binary system. It is generally assumed that the orbital energy of the binary is used to expel the envelope of the donor with an efficiency $\alpha_{\text {ce }}$ :

$$
E_{\text {bind }}=\alpha_{\text {ce }} \Delta E_{\text {orb }}
$$

where $E_{\text {bind }}$ is the total binding energy of the envelope and $\Delta E_{\text {orb }}$ is the orbital energy released in the spiral-in. What remains of the normal star $M_{1}$ is its stellar core $M_{\mathrm{c}}$. The above energy condition reads

$$
\frac{G M_{1}\left(M_{1}-M_{c}\right)}{\lambda R_{\mathrm{L}}}=\alpha_{\mathrm{ce}}\left(\frac{G m M_{c}}{2 a_{\mathrm{f}}}-\frac{G M_{1} m}{2 a_{\mathrm{i}}}\right)
$$

where $a_{\mathrm{i}}$ and $a_{\mathrm{f}}$ are the initial and the final orbital separations, and $\lambda$ is a numerical coefficient that depends on the structure of the donor's envelope. $R_{\mathrm{L}}$ is the Roche lobe radius of the normal star that can be approximated as [89]

$$
\frac{R_{\mathrm{L}}}{a_{\mathrm{i}}}=\frac{0.49}{0.6+q^{2 / 3} \ln \left(1+q^{1 / 3}\right)}
$$

and $q \equiv M_{1} / m$. From Equation (50) one derives

$$
\frac{a_{\mathrm{f}}}{a_{\mathrm{i}}}=\frac{M_{c}}{M_{1}}\left(1+\frac{2 a_{\mathrm{i}}}{\lambda \alpha_{\mathrm{ce}} R_{\mathrm{L}}} \frac{M_{1}-M_{c}}{m}\right)^{-1} \lesssim \frac{M_{c}}{M_{1}} \frac{M_{\mathrm{c}}}{\Delta M},
$$

where $\Delta M=M_{1}-M_{c}$ is the mass of the ejected envelope. The mass $M_{c}$ of a helium core of a massive star may be approximated as [415]

$$
M_{\mathrm{He}} \approx 0.1\left(M_{1} / M_{\odot}\right)^{1.4}
$$

so the orbital separation during the common envelope stage may decrease as much as by factor 100 and more.

The above formalism for the common envelope stage depends in fact on the product of two parameters: $\lambda$, which is the measure of the binding energy of the envelope to the core prior to mass transfer in a binary system, and $\alpha_{\text {ce }}$, which is the common envelope efficiency itself. Numerical calculations of evolved giant stars with masses $3-10 M_{\odot}$ [80] showed that the value of the $\lambda$ parameter is typically between 0.2 and 0.8 ; however, it can be as high as 5 on the asymptotic giant branch. For more massive primaries $\left(>20 M_{\odot}\right)$, which are appropriate for the formation of $\mathrm{BH}$ binaries, the $\lambda$-parameter was found to depend on the mass of the star and vary within a wide range $0.01-0.5$ [320]. Some hydrodynamical simulations [341] indicated that $\alpha_{\mathrm{ce}} \simeq 1$, while in others [367] a wider range for values of $\alpha_{\text {ce }}$ was obtained. There are debates in the literature as to should additional sources of energy (e.g., ionization energy in the envelope [134]) should be included in the ejection criterion of common envelopes [381].

There is another approach, different from the standard Webbink formalism, which is used to estimate the common envelope efficiency $\alpha_{\text {ce }}$. In the case of systems with at least one white-dwarf component one can try to reconstruct the evolution of double compact binaries with known masses of both components, since there is a unique relation between the mass of a white dwarf and the radius of its red giant progenitor. Close binary white dwarfs should definitely result from the spiral-in phase in the common envelope that appears inevitable during the second mass transfer (i.e. from the red giant to the white dwarf remnant of the original primary in the system). Such

Living Reviews in Relativity

http://www. livingreviews.org/lrr-2006-6 
an analysis [284], extended in [283, 431], suggests that the standard energy prescription for the treatment of the common envelope stage cannot be applied to the first mass transfer episode. Instead, the authors proposed to apply the so-called $\gamma$-formalism for the common envelope, in which not the energy but the angular momentum $J$ is balanced and conservation of energy is implicitly implied:

$$
\frac{\delta J}{J}=\gamma \frac{\Delta M}{M_{\mathrm{tot}}},
$$

where $\Delta M$ is the mass of the common envelope, $M_{\mathrm{tot}}$ is the total mass of the binary system before the common envelope, and $\gamma$ is a numerical coefficient. For all binary systems considered, the parameter $\gamma$ was found to be within the range 1.1-4, with the mean value around 1.5. (Notice that $\gamma=1$ corresponds to loss of the angular momentum through stellar wind, as considered above, which always increases the orbital separation of the binary.) The applicability of this algorithm should be investigated further.

Note also, that formulations of the common envelope equation different from Equation (50) are met in the literature (see, e.g., $[416,158]) ; a_{\mathrm{f}} / a_{\mathrm{i}}$ similar to the values produced by Equation (50) are then obtained for different $\alpha_{\mathrm{ce}} \lambda$ values. 


\section{Evolutionary Scenario for Compact Binaries with Neu- tron Star or Black Hole Components}

\subsection{Compact binaries with NSs}

Compact binaries with NS and BH components are descendants of initially massive binaries with $M_{1} \gtrsim(8-10) M_{\odot}$. The evolutionary scenario of massive binaries was elaborated shortly after the discovery of binary X-ray sources [415, 408, 427] and is depicted in Figure 4.

This scenario is fully confirmed by more than 30-years' history of astronomical observations and is now considered as standard. A massive X-ray binary is an inevitable stage preceding the formation of a double compact system after the second supernova explosion of the heliumrich companion in such a stellar system. The formation scenario for binary pulsars proposed immediately after the discovery of PSR 1913+16 [106, 253] has also been tested by subsequent observations of binary pulsars. In fact, the scenario for binary pulsars was proposed even earlier in [415], but because no binary pulsars were known at that time, it was suggested that all pairs of NS are disrupted at the second NS formation.

It is convenient to separate the evolution of a massive binary into several stages according to the physical state of the binary components, including phases of mass exchange between them. The simplest evolutionary scenario can be schematically described as follows (see Figure 4).

1. Initially, two high-mass OB main-sequence stars are separated and are inside their Roche lobes. Tidal interaction is very effective so that a possible initial eccentricity vanishes before the primary star $M_{1}$ fills its Roche lobe. The duration of this stage is determined by the hydrogen burning time of the primary and typically is several million years (for massive main-sequence stars, the time of core hydrogen burning is $t_{\text {nucl }} \propto M^{-2}$ ). The star burns out hydrogen in its central parts, so that a dense central helium core with a mass $M_{\mathrm{He}} \simeq$ $0.1\left(M / M_{\odot}\right)^{1.4}$ forms by the time when the star leaves the main sequence. The expected number of such binaries in the Galaxy is around $10^{4}$.

2. After core hydrogen exhaustion, the primary leaves the main sequence and starts to expand rapidly. When its radius approaches the Roche lobe (see Equation (51)), mass transfer onto the secondary, less massive star which still resides on the main sequence begins. The masstransfer rate can be crudely estimated as $\dot{M} \sim M_{1} / \tau_{\mathrm{KH}}$, where $\tau_{\mathrm{KH}}=G M_{1}^{2} / R_{1} L_{1}$ is the primary's thermal time scale.

The mass transfer ends when most of the primary's hydrogen envelope is transferred onto the secondary, so a naked helium core is left. This core can be observed as a Wolf-Rayet (WR) star with intense stellar wind if its mass exceeds $(7-8) M_{\odot}[293,94,95]$.

While the mass of the primary star reduces, the mass of the secondary star increases, since the mass transfer at this stage is thought to be quasi-conservative. For not too massive main-sequence stars, $M \lesssim 40 M_{\odot}$, no significant stellar wind mass loss occurs which could, otherwise, remove too much matter from the binary, thereby increasing binary separation. The secondary star acquires large angular momentum due to the infalling material, so that its outer envelope can be spun up to an angular velocity close to the limiting (Kepler orbit) value. Such massive rapidly rotating stars are observed as Be-stars. During the conservative stage of mass transfer, the semimajor axis of the orbit first decreases, reaches a minimum when the masses of the binary components become equal to each other, and then increases. This behavior is dictated by the angular momentum conservation law (31). After the completion of the conservative mass transfer, the initially more massive star becomes less massive than its initially lighter companion.

Living Reviews in Relativity

http: //www . livingreviews . org/lrr-2006-6 


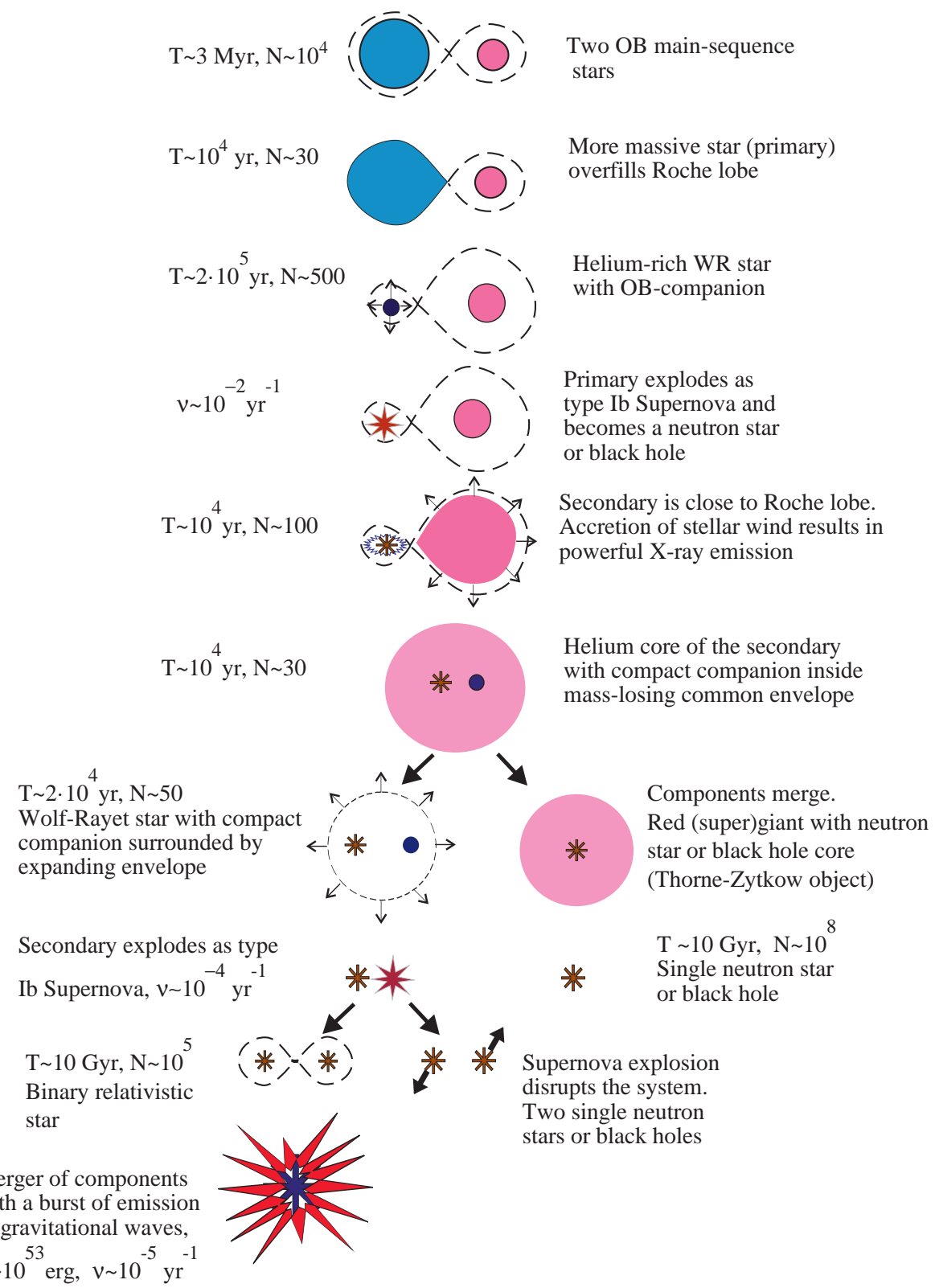

Figure 4: Evolutionary scenario for the formation of neutron stars or black holes in close binaries. 
For the typical parameters the duration of the first RLOF is rather short, of the order of $10^{4} \mathrm{yr}$, so only several dozens of such binaries are expected to be in the Galaxy.

3. The duration of the WR stage is about several $10^{5} \mathrm{yr}$, so the Galactic number of such binaries should be several hundreds.

4. At the end of the thermonuclear evolution, the WR star explodes as Ib (or Ic) supernova to become a NS or $\mathrm{BH}$. The inferred Galactic type Ib SN rate is around $10^{-2}$ per year, at least half of them should be in binaries. At this stage the disruption of the binary is possible (e.g., if the mass lost during the symmetric SN explosion exceeds $50 \%$ of the total mass of the pre-SN binary, or even smaller in the presence of the kick velocity; see Section 3.3 above). Some runaway Galactic OB-stars must have been formed in this way.

5. If the system survives the first SN explosion, a rapidly rotating Be star in pair with a young NS appears. Orbital evolution following the SN explosion is described above by Equations (4045). The orbital eccentricity after the SN explosion is high, so enhanced accretion onto the NS occurs at the periastron passages. Most of about 100 Galactic Be/X-ray binaries [335] are formed in this way. The duration of this stage depends on the binary parameters, but in all cases it is limited by the time left for the (now more massive) secondary to burn hydrogen in its core.

An important parameter of NS evolution is the surface magnetic field strength. In binary systems, magnetic field, in combination with NS spin period and accretion rate onto the NS surface, determines the observational manifestation of the neutron star (see [219] for more detail). Accretion of matter onto the NS can reduce the surface magnetic field and spin-up the NS rotation (pulsar recycling) [37, 356, 357, 36].

6. The secondary expands to engulf the NS. The common envelope stage begins and after $\sim 10^{3}$ yr ends up with the formation of a WR star with a compact companion surrounded by an expanding envelope (Cyg X-3 may serve as an example), or the NS merges with the helium core during the common envelope to form a still hypothetic Thorne-Żytkow (TZ) object. The fate of TZ stars remains unclear (see [17] for the recent study). Single (possibly, massive) NS or BH should descend from them.

A note should be made concerning the phase when a common envelope engulfs the firstformed NS and the core of the secondary. Colgate [60] and Zel'dovich et al. [475] have shown that hyper-Eddington accretion onto a neutron star is possible if the gravitational energy released in accretion is lost by neutrinos. Chevalier [55] suggested that this may be the case for the accretion in common envelopes. Since the accretion rates in this case may be as high as $\sim 0.1 M_{\odot} \mathrm{yr}^{-1}$, the NS may collapse into a $\mathrm{BH}$ inside the common envelope. An essential caveat is that the accretion in the hyper-Eddington regime may be prevented by the angular momentum of the captured matter. The magnetic field of the NS may also be a complication. The possibility of hyper-critical accretion still has to be studied. Nevertheless, implications of this hypothesis for different types of relativistic binaries were explored in great detail by H. Bethe and G. Brown and their coauthors (see, e.g., [44] and references therein). Also, the possibility of hyper-Eddington accretion was included in several population synthesis studies with evident result of diminishing the population of NS + NS binaries in favour of neutron stars in pairs with low-mass black holes (see, e.g., [328, 22]).

7. The secondary WR ultimately explodes as a type Ib supernova leaving behind a double NS binary, or the system is disrupted to form two single high-velocity NSs or BHs. Even for a symmetric SN explosion the disruption of binaries after the second SN explosion could result in the observed high average velocities of radiopulsars (see Section 3.4 above). In the 
surviving close binary NS system, the older NS is expected to have faster rotation velocity (and possibly higher mass) than the younger one because of the recycling at the preceding accretion stage. The subsequent orbital evolution of such double NS systems is entirely due to GW emission (see Section 3.1.4) and ultimately leads to the coalescence of the components.

Detailed studies of possible evolutionary channels which produce merging binary NS can be found in the literature (see, e.g., [419, 420, 227, 328, 13, 449, 22, 169, 81, 453]).

We emphasize that this scenario applies only to initially massive binaries. There exists also a population of NSs accompanied by low-mass $\left[\sim(1-2) M_{\odot}\right]$ companions. A scenario similar to the one presented in Figure 4 may be sketched for them too, with the difference that the secondary component stably transfers mass onto the companion (see, e.g., [165, 183, 184, 410]). This scenario is similar to the one for low- and intermediate-mass binaries considered in Section 7, with the WD replaced by a NS or a BH. Compact low-mass binaries with NSs may be dynamically formed in dense stellar environments, for example in globular clusters. The dynamical evolution of binaries in globular clusters is beyond the scope of this review; see [26] and [36] for more detail and further references.

\subsection{Black hole formation parameters}

So far, we have considered the formation of NSs and binaries with NSs. It is believed that very massive stars end up their evolution with the formation of stellar mass black holes. We will discuss now their formation.

In the analysis of $\mathrm{BH}$ formation, new important parameters appear. The first one is the threshold mass $M_{\mathrm{cr}}$ beginning from which a main-sequence star, after the completion of its nuclear evolution, can collapse into a BH. This mass is not well known; different authors suggest different values: van den Heuvel and Habets [428] $-40 M_{\odot}$; Woosley et al. [458] $-60 M_{\odot}$; Portegies Zwart, Verbunt, and Ergma [327] - more than $20 M_{\odot}$. A simple physical argument usually put forward in the literature is that the mantle of the main-sequence star with $M>M_{\mathrm{cr}} \approx 30 M_{\odot}$ before the collapse has a binding energy well above $10^{51}$ erg (the typical supernova energy observed), so that the supernova shock is not strong enough to expel the mantle [110, 111].

The upper mass limit for $\mathrm{BH}$ formation (with the caveat that the role of magnetic-field effects is not considered) is, predominantly, a function of stellar-wind mass loss in the core-hydrogen, hydrogen-shell, and core-helium burning stages. For a specific combination of winds in different evolutionary stages and assumptions on metallicity it is possible to find the types of stellar remnants as a function of initial mass (see, for instance [138]). Since stellar winds are mass (or luminosity) and metallicity-dependent, a peculiar consequence of mass-loss implementation in the latter study is that for $Z \simeq Z_{\odot}$ the mass-range of precursors of black holes is constrained to $M \approx(25-60) M_{\odot}$, while more massive stars form NSs because of heavy mass loss. The recent discovery of the possible magnetar in the young stellar cluster Westerlund 1 [263] hints to the reality of such a scenario. Note, however, that the estimates of $\dot{M}$ are rather uncertain, especially for the most massive stars, mainly because of clumping in the winds (see, e.g., [201, 69, 129]). Current reassessment of the role of clumping generally results in the reduction of previous mass-loss estimates. Other factors that have to be taken into account in the estimates of the masses of progenitors of BHs are rotation and magnetic fields.

The second parameter is the mass $M_{\mathrm{BH}}$ of the nascent $\mathrm{BH}$. There are various studies as for what the mass of the $\mathrm{BH}$ should be (see, e.g., $[403,32,110,113]$ ). In some papers a typical $\mathrm{BH}$ mass was found to be not much higher than the upper limit for the NS mass (Oppenheimer-Volkoff limit $\sim(1.6-2.5) M_{\odot}$, depending on the unknown equation of state for NS matter) even if the fallback accretion onto the supernova remnant is allowed [403]. Modern measurements of black hole masses in binaries suggest a broad range of $\mathrm{BH}$ masses of the order of $4-17 M_{\odot}[296,254,345]$. A continuous range of $\mathrm{BH}$ masses up to $10-15 M_{\odot}$ was derived in calculations [113]. Since present 
day calculations are still unable to reproduce self-consistently even the supernova explosion, in the further discussion we have parameterized the $\mathrm{BH}$ mass $M_{\mathrm{BH}}$ by the fraction of the pre-supernova mass $M_{*}$ that collapses into the $\mathrm{BH}: k_{\mathrm{BH}}=M_{\mathrm{BH}} / M_{*}$. In fact, the pre-supernova mass $M_{*}$ is directly related to $M_{\mathrm{cr}}$, but the form of this relationship is somewhat different in different scenarios for massive star evolution, mainly because of different mass-loss prescriptions. According to our parameterization, the minimal $\mathrm{BH}$ mass can be $M_{\mathrm{BH}}^{\mathrm{min}}=k_{\mathrm{BH}} M_{*}$, where $M_{*}$ itself depends on $M_{\mathrm{cr}}$. The parameter $k_{\mathrm{BH}}$ can vary in a wide range.

The third parameter, similar to the case of NS formation, is the possible kick velocity $\mathbf{w}_{\mathrm{BH}}$ imparted to the newly formed BH (see the end of Section 3.4). In general, one expects that the $\mathrm{BH}$ should acquire a smaller kick velocity than a NS, as black holes are more massive than neutron stars. A possible relation (as adopted, e.g., in calculations [227]) reads

$$
\frac{w_{\mathrm{BH}}}{w_{\mathrm{NS}}}=\frac{M_{*}-M_{\mathrm{BH}}}{M_{*}-M_{\mathrm{OV}}}=\frac{1-k_{\mathrm{BH}}}{1-M_{\mathrm{OV}} / M_{*}},
$$

where $M_{\mathrm{OV}}=2.5 M_{\odot}$ is the maximum NS mass. When $M_{\mathrm{BH}}$ is close to $M_{\mathrm{OV}}$, the ratio $w_{\mathrm{BH}} / w_{\mathrm{NS}}$ approaches 1, and the low-mass black holes acquire kick velocities similar to those of neutron stars. When $M_{\mathrm{BH}}$ is significantly larger than $M_{\mathrm{OV}}$, the parameter $k_{\mathrm{BH}}=1$, and the $\mathrm{BH}$ kick velocity becomes vanishingly small. The allowance for a quite moderate $w_{\mathrm{BH}}$ can increases the coalescence rate of binary $\mathrm{BH}[227]$.

The possible kick velocity imparted to newly born black holes makes the orbits of survived systems highly eccentric. It is important to stress that some fraction of such binary BH can retain their large eccentricities up to the late stages of their coalescence. This signature should be reflected in their emitted waveforms and should be modeled in templates.

Asymmetric explosions accompanied by a kick change the space orientation of the orbital angular momentum. On the other hand, the star's spin axis remains fixed (unless the kick was off-center). As a result, some distribution of the angles between the BH spins and the orbital angular momentum (denoted by $J$ ) will be established [330]. It is interesting that even for small kicks of a few tens of $\mathrm{km} / \mathrm{s}$ an appreciable fraction $(30-50 \%)$ of the merging binary $\mathrm{BH}$ can have $\cos J<0$. This means that in these binaries the orbital angular momentum vector is oriented almost oppositely to the black hole spins. This is one more signature of imparted kicks that can be tested observationally. These effects are also discussed in [179]. 


\section{Formation of Double Compact Binaries}

\subsection{Analytical estimates}

A rough estimate of the formation rate of double compact binaries can be obtained ignoring many details of binary evolution. To do this, we shall use the observed initial distribution of binary orbital parameters and assume the simplest conservative mass transfer $\left(M_{1}+M_{2}=\right.$ const) without kick velocity imparted to the nascent compact stellar remnants during SN explosions.

Initial binary distributions. From observations of spectroscopic binaries it is possible to derive the formation rate of binary stars with initial masses $M_{1}, M_{2}$ (with mass ratio $q=M_{2} / M_{1} \leq 1$ ), orbital semimajor axis $A$, and eccentricity $e$. According to [326], the present birth rate of binaries in our Galaxy can be written in factorized form as

$$
\frac{d N}{d A d M_{1} d q d t} \approx 0.087\left(\frac{A}{R_{\odot}}\right)^{-1}\left(\frac{M_{1}}{M_{\odot}}\right)^{-2.5} f(q),
$$

where $f(q)$ is a poorly constrained distribution over the initial mass ratio of binary components.

One usually assumes a mass ratio distribution law in the form $f(q) \sim q^{-\alpha_{q}}$ where $\alpha_{q}$ is a free parameter; another often used form of the $q$-distribution was suggested by Kuiper [202]:

$$
f(q)=2 /(1+q)^{2} .
$$

The range of $A$ is $10 \leq A / R_{\odot} \leq 10^{6}$. In deriving the above Equation (56), the authors of [326] took into account selection effects to convert the "observed" distribution of stars into the true one. An almost flat logarithmic distribution of semimajor axes was also found in [4]. Integration of Equation (56) yields one binary system with $M_{1} \gtrsim 0.8 M_{\odot}$ and $10 R_{\odot}<A<10^{6} R_{\odot}$ per year in the Galaxy, which is in reasonable agreement with the Galactic star formation rate estimated by various methods; the present-day star formation rate is about several $M_{\odot}$ per year (see, for example, [255, 401]).

Constraints from conservative evolution. To form a NS at the end of thermonuclear evolution, the primary mass should be at least $10 M_{\odot}$. Equation (56) says that the formation rate of such binaries is about 1 per 50 years. We shall restrict ourselves by considering only close binaries, in which mass transfer onto the secondary is possible. This narrows the binary separation interval to $10-1000 R_{\odot}$ (see Figure 1$)$; the birth rate of close massive $\left(M_{1}>10 M_{\odot}\right)$ binaries is thus $1 / 50 \times 2 / 5 \mathrm{yr}^{-1}=1 / 125 \mathrm{yr}^{-1}$. The mass ratio $q$ should not be very small to make the formation of the second NS possible. The lower limit for $q$ is derived from the condition that after the first mass transfer stage, during which the mass of the secondary increases, $M_{2}+\Delta M \geq 10 M_{\odot}$. Here $\Delta M=M_{1}-M_{\mathrm{He}}$ and the mass of the helium core left after the first mass transfer is $M_{\mathrm{He}} \approx 0.1\left(M_{1} / M_{\odot}\right)^{1.4}$. This yields

$$
m_{2}+\left(m_{1}-0.1 m_{1}^{1.4}\right)>10,
$$

where we used the notation $m=M / M_{\odot}$, or in terms of $q$ :

$$
q \geq 10 / m_{1}+0.1 m_{1}^{0.4}-1 .
$$

An upper limit for the mass ratio is obtained from the requirement that the binary system remains bound after the sudden mass loss in the second supernova explosion ${ }^{5}$. From Equation (45) we obtain

$$
\frac{0.1\left[m_{2}+\left(m_{1}-0.1 m_{1}^{1.4}\right)\right]^{1.4}-1.4}{2.8}<1
$$

\footnotetext{
${ }^{5}$ For the first supernova explosion without kick this is always satisfied.
} 
or in terms of $q$ :

$$
q \leq 14.4 / m_{1}+0.1 m_{1}^{0.4}-1 .
$$

Inserting $m_{1}=10$ in the above two equations yields the appropriate mass ratio range $0.25<q<$ 0.69 , i.e. $20 \%$ of the binaries for Kuiper's mass ratio distribution. So we conclude that the birth rate of binaries which potentially can produce double NS system is $\lesssim 0.2 \times 1 / 125 \mathrm{yr}^{-1} \simeq 1 / 600 \mathrm{yr}^{-1}$.

Of course, this is a very crude upper limit - we have not taken into account the evolution of the binary separation, ignored initial binary eccentricities, non-conservative mass loss, etc. However, it is not easy to treat all these factors without additional knowledge of numerous details and parameters of binary evolution (such as the physical state of the star at the moment of the Roche lobe overflow, the common envelope efficiency, etc.). All these factors should decrease the formation rate of double NS. The coalescence rate of compact binaries (which is ultimately of interest for us) will be even smaller - for the compact binary to merge within the Hubble time, the binary separation after the second supernova explosion should be less than $\sim 100 R_{\odot}$ (orbital periods shorter than $\sim 40 \mathrm{~d}$ ) for arbitrary high orbital eccentricity $e$ (see Figure 3 ). The model-dependent distribution of NS kick velocities provides another strong complication. We also stress that this upper limit was obtained assuming a constant Galactic star-formation rate and normalization of the binary formation by Equation (56).

Further (semi-)analytical investigations of the parameter space of binaries leading to the formation of coalescing binary NSs are still possible but technically very difficult, and we shall not reproduce them here. The detailed semi-analytical approach to the problem of formation of NSs in binaries and evolution of compact binaries has been developed by Tutukov and Yungelson [419, 420].

\subsection{Population synthesis results}

A distinct approach to the analysis of binary star evolution is based on the population synthesis method - a Monte-Carlo simulation of the evolution of a sample of binaries with different initial parameters. This approach was first applied to model various observational manifestations of magnetized NSs in massive binary systems [195, 196, 78] and generalized to binary systems of arbitrary mass in [224] (The Scenario Machine code). To achieve a sufficient statistical significance, such simulations usually involve a large number of binaries, typically of the order of a million. The total number of stars in the Galaxy is still four orders of magnitude larger, so this approach cannot guarantee that rare stages of the binary evolution will be adequately reproduced ${ }^{6}$.

Presently, there are several population synthesis codes used for massive binary system studies, which take into account with different degree of completeness various aspects of binary stellar evolution (e.g., the codes by Portegies Zwart et al. [328, 469], Bethe and Brown [32], Hurley, Tout, and Pols [154], Belczynski et al. [23], Yungelson and Tutukov [423]). A review of applications of the population synthesis method to various types of astrophysical sources and further references can be found in [325, 467]. Some results of population synthesis calculations of compact binary mergers carried out by different groups are presented in Table 4.

Actually, the authors of the studies mentioned in Table 4 make their simulations for a range of parameters. We list in the table the rates for the models which the authors themselves consider as "standard" or "preferred" or "most probable". Generally, for the NS + NS merger rate Table 4 shows the scatter within a factor $\sim 4$, which may be considered quite reasonable, having in mind the uncertainties in input parameters. There are two clear outliers, [420] and [437]. The high rate in [420] is due to the assumption that kicks to nascent neutron stars are absent. The low rate in [437] is due to the fact that these authors apply in the common envelope equation an evolutionarystage-dependent structural constant $\lambda$. Their range for $\lambda$ is $0.006-0.4$, to be compared with the

\footnotetext{
${ }^{6}$ Instead of Monte-Carlo simulations one may use a sufficiently dense grid in the 3D space of binary parameters and integrate over this grid (see, e.g., [423] and references therein).
}

Living Reviews in Relativity

http: //www. livingreviews.org/lrr-2006-6 


\begin{tabular}{l|rcrr}
\hline \hline Authors & Ref. & $\begin{array}{c}\mathrm{NS}+\mathrm{NS} \\
{\left[\mathrm{yr}^{-1}\right]}\end{array}$ & $\begin{array}{c}\mathrm{NS}+\mathrm{BH} \\
{\left[\mathrm{yr}^{-1}\right]}\end{array}$ & $\begin{array}{c}\mathrm{BH}+\mathrm{BH} \\
{\left[\mathrm{yr}^{-1}\right]}\end{array}$ \\
\hline Tutukov and Yungelson (1993) & {$[420]$} & $3 \times 10^{-4}$ & $2 \times 10^{-5}$ & $1 \times 10^{-6}$ \\
Lipunov et al. (1997) & {$[226]$} & $3 \times 10^{-5}$ & $2 \times 10^{-6}$ & $3 \times 10^{-7}$ \\
Portegies Zwart and Yungelson (1998) & {$[328]$} & $2 \times 10^{-5}$ & $10^{-6}$ & \\
Nelemans et al. (2001) & {$[285]$} & $2 \times 10^{-5}$ & $4 \times 10^{-6}$ & \\
Voss and Tauris (2003) & {$[437]$} & $2 \times 10^{-6}$ & $6 \times 10^{-7}$ & $10^{-5}$ \\
O'Shaughnessy et al. (2005) & {$[297]$} & $7 \times 10^{-6}$ & $1 \times 10^{-6}$ & $1 \times 10^{-6}$ \\
de Freitas Pacheco et al. (2006) & {$[71]$} & $2 \times 10^{-5}$ & & \\
\hline \hline
\end{tabular}

Table 4: Examples of the estimates for Galactic merger rates of relativistic binaries calculated under different assumptions on the parameters entering population synthesis.

"standard" $\lambda=0.5$ applied in most of the other studies. A low $\lambda$ favours mergers in the first critical lobe overflow episode and later mergers of the first-born neutron stars with their non-relativistic companions $^{7}$. A considerable scatter in the rates of mergers of systems with $\mathrm{BH}$ companions is due, mainly, to uncertainties in stellar wind mass loss for the most massive stars. For instance, the implementation of winds in the code used in $[328,285]$ resulted in the absence of merging $\mathrm{BH}+\mathrm{BH}$ systems, while a rather low $\dot{M}$ assumed in [437] produced a high merger rate of $\mathrm{BH}+\mathrm{BH}$ systems.

A word of caution should be said here. It is hardly possible to trace a detailed evolution of each binary, so one usually invokes the approximate approach to describe the change of evolutionary stages of the binary components (the so-called evolutionary track), their interaction, effects of supernovae, etc. Thus, fundamental uncertainties of stellar evolution mentioned above are complemented with (i) uncertainties of the scenario and (ii) uncertainties in the normalization of the calculations to the real galaxy (such as the fraction of binaries among allstars, the star formation history, etc.). The intrinsic uncertainties in the population synthesis results (for example, in the computed event rates of binary mergers etc.) are in the best case not less than of the order of factor two or three. This should always be born in mind when using the population synthesis calculations. However, we emphasize again the fact that the double NS merger rate, as inferred from binary pulsar statistics with account for the double pulsar observations [48, 180], is very close to the population syntheses estimates with a kick of about $(250-300) \mathrm{km} \mathrm{s}^{-1}$.

\footnotetext{
${ }^{7}$ Note that similar low values of $\lambda$ for 20 to $50 M_{\odot}$ stars were obtained also by [320]. If confirmed, these results may have major impact on the estimates of merger rates for relativistic binaries.
} 


\section{Detection Rates}

The detection of a gravitational wave signal from merging close binaries is characterized by the signal-to-noise ratio $S / N$, which depends on the binary masses, the distance to the binary, the frequency, and the noise characteristics. A pedagogical derivation of the signal-to-noise ratio and its discussion for different detectors is given, for example, in Section 8 of the review [122].

In this section we focus of two particular points: the plausible enhancement of the detection of merging binary black holes with respect to binary neutron stars and the way how absolute detection rates of binary mergings can be calculated.

\subsection{Enhancement of the detection rate for binary $\mathrm{BH}$ mergers}

Coalescing binaries emit gravitational wave signals with a well known time-dependence (waveform) (see Section 3.1 above). This allows one to use the technique of matched filtering [399]. The signalto-noise ratio $S / N$ for a particular detector, which is characterized by the dimensionless noise rms amplitude $h_{\text {rms }}$ at a given frequency $f$, depends mostly on the "chirp" mass of the binary system $\mathcal{M}=\left(M_{1}+M_{2}\right)^{-1 / 5}\left(M_{1} M_{2}\right)^{3 / 5}$ and its distance $r$. Here, we will use the simplified version for $S / N([399] ;$ see also [105]):

$$
\frac{S}{N}=3^{-1 / 2} \pi^{-2 / 3} \frac{G^{5 / 6}}{c^{3 / 2}} \frac{\mathcal{M}^{5 / 6}}{r} \frac{f^{-1 / 6}}{h_{\mathrm{rms}}(f)} .
$$

At a fixed level of $S / N$, the detection volume is proportional to $r^{3}$ and therefore it is proportional to $\mathcal{M}^{5 / 2}$. The detection rate $\mathcal{D}$ for binaries of a given class $(\mathrm{NS}+\mathrm{NS}, \mathrm{NS}+\mathrm{BH}$ or $\mathrm{BH}+\mathrm{BH})$ is the product of their coalescence rate $\mathcal{R}_{\mathrm{V}}$ with the detector's registration volume $\propto \mathcal{M}^{5 / 2}$ for these binaries.

It is seen from Table 4 that the model Galactic rate $\mathcal{R}_{\mathrm{G}}$ of $\mathrm{NS}+\mathrm{NS}$ coalescences is typically higher than the rate of $\mathrm{NS}+\mathrm{BH}$ and $\mathrm{BH}+\mathrm{BH}$ coalescences. However, the $\mathrm{BH}$ mass can be significantly larger than the NS mass. So a binary involving one or two black holes, placed at the same distance as a NS + NS binary, produces a significantly larger amplitude of gravitational waves. With the given sensitivity of the detector (fixed $S / N$ ratio), a $\mathrm{BH}+\mathrm{BH}$ binary can be seen at a greater distance than a NS + NS binary. Hence, the registration volume for such bright binaries is significantly larger than the registration volume for relatively weak binaries. The detection rate of a given detector depends on the interplay between the coalescence rate and the detector's response to the sources of one or another kind.

If we assign some characteristic (mean) chirp mass to different types of double NS and $\mathrm{BH}$ systems, the expected ratio of their detection rates by a given detector is

$$
\frac{\mathcal{D}_{\mathrm{BH}}}{\mathcal{D}_{\mathrm{NS}}}=\frac{\mathcal{R}_{\mathrm{BH}}}{\mathcal{R}_{\mathrm{NS}}}\left(\frac{\mathcal{M}_{\mathrm{BH}}}{\mathcal{M}_{\mathrm{NS}}}\right)^{5 / 2}
$$

where $\mathcal{D}_{\mathrm{BH}}$ and $\mathcal{D}_{\mathrm{NS}}$ refer to $\mathrm{BH}+\mathrm{BH}$ and $\mathrm{NS}+\mathrm{NS}$ pairs, respectively. Here, we discuss the ratio of the detection rates, rather than their absolute values. The derivation of absolute values requires detailed evolutionary calculations, as we discussed above. Taking $\mathcal{M}_{\mathrm{BH}}=8.7 M_{\odot}$ (for $\left.10 M_{\odot}+10 M_{\odot}\right)$ and $\mathcal{M}_{\mathrm{NS}}=1.22 M_{\odot}\left(\right.$ for $\left.1.4 M_{\odot}+1.4 M_{\odot}\right)$, Equation $(60)$ yields

$$
\frac{\mathcal{D}_{\mathrm{BH}}}{\mathcal{D}_{\mathrm{NS}}} \approx 140 \frac{\mathcal{R}_{\mathrm{BH}}}{\mathcal{R}_{\mathrm{NS}}} .
$$

As $\frac{\mathcal{R}_{\mathrm{BH}}}{\mathcal{R}_{\mathrm{NS}}}$ is typically $0.1-0.01$ (see Table 4), this relation suggests that the registration rate of $\mathrm{BH}$ mergers can be higher than that of NS mergers. This estimate is, of course, very rough, but it can serve as an indication of what one can expect from detailed calculations. We stress that the effect of an enhanced detection rate of $\mathrm{BH}$ binaries is independent of the desired $S / N$ and other characteristics of the detector; it was discussed, for example, in [420, 226, 122]. 


\subsection{Note on realistic calculation of the detection rates of binary merg- ings}

Now we shall briefly discuss how the detection rates of binary mergings can be calculated for a given gravitational wave detector. For a secure detection, the $S / N$ ratio is usually raised up to $7-8$ to avoid false alarms over a period of a year (assuming Gaussian noise) ${ }^{8}$. This requirement determines the maximum distance from which an event can be detected by a given interferometer. The distance ranges of LIGO I/VIRGO (LIGO II) interferometers for relativistic binary inspirals are given in [70]: $20(300) \mathrm{Mpc}$ for $\mathrm{NS}+\mathrm{NS}\left(1.4 M_{\odot}+1.4 M_{\odot}\right), 43(650) \mathrm{Mpc}$ for $\mathrm{NS}+\mathrm{BH}\left(1.4 M_{\odot}+10 M_{\odot}\right)$ Note that the distances increase for a network of detectors.

To calculate a realistic detection rate of binary mergers the distribution of galaxies should be taken into account within the volume bounded by the distance range of the detector (see, for example, the earlier attempt to take into account only bright galaxies from Tully's catalog of nearby galaxies in [221], and the use of LEDA database of galaxies to estimate the detection rate of supernova explosions [19]). However, not only the mass and type of a given galaxy, but also the star formation rate and, better, the history of the star formation rate in that galaxy (since the binary merger rate in galaxies strongly evolves with time [229]) are needed to estimate the expected detection rate $\mathcal{D}$. But this is a tremendous problem - even the sample of galaxies (mostly, dwarfs) within the Local Volume $(<8 \mathrm{Mpc})$ is only $70 \%-80 \%$ complete [185], and the number of new nearby galaxies continues to increase. So to assess the merger rate from a large volume based on the Galactic values, the best one can do at present appears to be using formulas like Equation (4) given earlier in Section 2.2. This, however, adds another factor two of uncertainty in the estimates. Clearly, a more accurate treatment of the transition from Galactic rates to larger volumes with an account of the galaxy distribution is required.

\subsection{Going further}

The most important future observations include:

1. Routine increase of the statistics of binary pulsars, especially with low radio luminosity. This will allow one to put stronger constraints on the NS + NS merger rate as directly inferred from the binary pulsars statistics. More indirectly, a larger sample of NS parameters in binary pulsars would be useful for constraining the range of parameters of scenarios of formation for double NSs and, hence, a better understanding their origin (see, for example, a recent attempt of such an analysis in [297]).

2. Discovery of a possible NS $+\mathrm{BH}$ binary. Measurements of its parameters would be crucial for models of formation and evolution of BHs in binary systems in general. Current estimates of the number of such binaries in the Galaxy, obtained by the population synthesis method, range from one per several thousand ordinary pulsars $[228,220]$ to much smaller values of about $0.1-1 \%$ of the number of double NSs in the Galactic disk [309].

3. Search for unusual observational manifestations of relativistic binaries (e.g., among some new radio transient sources like GCRT J1745-3009, firm identifications with some GRBs, etc.)

4. Improving the estimates of binary merger rate limits from data taken by GW detectors.

The most important theoretical issues include:

1. Stellar physics: post-helium burning evolution of massive stars, supernova explosion mechanism, masses of compact stars formed in the collapse, mechanism(s) of kick velocity imparted

${ }^{8}$ If a network of three detectors, such as two LIGOs and VIRGO, runs simultaneously, the $S / N$ ratio in an individual detector should be $>7 / \sqrt{3} \approx 4$ ) 
to nascent compact remnants (neutron stars and black holes), stellar winds from hydrogenand helium-rich stars.

2. Binary evolution: treatment of the common envelope stage, magnetic braking for low-mass binaries, observational constraints on the initial distributions of orbital parameters of binary stars (masses, semimajor axes, eccentricities).

3. Last but not least, it is very important to improve our knowledge of such "traditional" topics of stellar astronomy as the fraction of binary stars among the total population, distributions of binary stars over separations of components, and their mass ratios. 


\section{Formation of Short-Period Binaries with a White-Dwarf Components}

Binary systems with white dwarf components that are interesting for general relativity and cosmology come in several flavours:

- Detached binary white dwarfs or "double degenerates" (DDs, we shall use both terms as synonyms below).

- Cataclysmic variables (CVs) - a class of semidetached binary stars containing a white dwarf and a companion star that is usually a red dwarf or a slightly evolved star, a subgiant.

- A subclass of the former systems in which the Roche lobe is filled by another white dwarf or low-mass partially degenerate helium star (AM CVn-type stars or "interacting doubledegenerates", IDDs). They appear to be important LISA verification sources.

- Detached systems with a white dwarf accompanied by a low-mass nondegenerate helium star (SD + WD systems).

- Ultracompact X-ray binaries (UCXBs) where one of the components is a NS, while the Roche lobe overflowing component is a WD.

As Figure 2 shows, compact binary stars emit gravitational waves within the sensitivity limits for space-based detectors if their orbital periods are from $\sim 20 \mathrm{~s}$ to $20,000 \mathrm{~s}$. This means that in principle all AM CVn-stars, UCXBs, a considerable fraction of all CVs with measured orbital periods, and some DD and SD + WD systems would be observable in GWs in the absence of confusion noise and sufficient sensitivity of detectors.

Though general relativity predicted that binary stars have to be the sources of gravitational waves in the 1920 s, this prediction became a matter of actual interest only with the discovery of the $P_{\text {orb }} \approx 81.5$ min cataclysmic variable WZ Sge by Kraft, Mathews, and Greenstein in 1962 [198], who immediately recognized the significance of short-period binary stars as testbeds for gravitational waves physics. Another impetus to the study of binaries as sources of gravitational wave radiation (GWR) was imparted by the discovery of the ultra-short period binary HZ $29=$ AM CVn $\left(P_{\text {orb }} \approx\right.$ 18 min) by Smak in 1967 [379]. Smak [379] and Paczyński [300] speculated that the latter system is a close pair of white dwarfs, without specifying whether it is detached or semidetached. Faulkner et al. [100] inferred the status of AM CVn as a "double-white-dwarf semidetached" nova. AM CVn was later classified as a cataclysmic variable after flickering typical for CVs was found for AM CVn by Warner and Robinson $[442]^{9}$ and became a prototype of a subclass of binaries ${ }^{10}$.

The origin of all above mentioned classes of short-period binaries was understood after the notion of common envelopes and the formalism for their treatment were suggested in 1970s (see Section 3.5). A spiral-in of components in common envelopes allowed to explain how white dwarfs - former cores of highly evolved stars with radii of $\sim 100 R_{\odot}$ - may acquire companions separated by $\sim R_{\odot}$ only (for pioneering work see [301, 443, 417, 418, 160, 444]). We recall, however, that most studies of the formation of compact objects through common envelopes are based on a simple formalism of comparison of binding energy of the envelope with the orbital energy of the binary, thought to be the sole source of energy for the loss of the envelope as described in Section 3.5. Though full-scale hydrodynamic calculations of a common-envelope evolution exist, for instance a

\footnotetext{
${ }^{9}$ Flickering is a fast intrinsic brightness scintillation occurring on time scales from seconds to minutes with amplitudes of $0.01-1 \mathrm{mag}$ and suggesting an ongoing mass transfer.

${ }^{10}$ Remarkably, a clear-cut confirmation of the binary nature of AM CVn and the determination of its true period awaited for almost 25 years [281].
} 
series of papers by Taam and coauthors published over more than two decades (see [368, 367] and references therein), the process is still very far from comprehension.

We recall also that the stability and timescale of mass-exchange in a binary depends on the mass ratio of components $q$, the structure of the envelope of Roche-lobe filling star, and possible stabilizing effects of mass and momentum loss from the system [413, 144, 470, 127, 135, 171, 102, 47]. For stars with radiative envelopes, to the first approximation, mass exchange is stable if $q \lesssim 1.2$; for $1.2 \lesssim q \lesssim 2$ it proceeds in the thermal time scale of the donor; for $q \gtrsim 2$ it proceeds in the dynamical time scale. Mass loss occurs on a dynamical time scale if the donor has a deep convective envelope or if it is degenerate and conditions for stable mass exchange are not satisfied. It is currently commonly accepted, despite a firm observational proof is lacking, that the distribution of binaries over $q$ is even or rises to small $q$ (see Section 5). Since typically the accretion rate is limited either by the rate that corresponds to the thermal time scale of the accretor or its Eddington accretion rate, both of which are lower than the mass-loss rate by the donor, the overwhelming majority $(\sim 90 \%)$ of close binaries pass in their evolution through one to four stages of a common envelope.

An "initial donor mass - donor radius at RLOF" diagram showing descendants of stars after mass-loss in close binaries is presented in Figure 1. We should remember here that solar metallicity stars with $M \lesssim 0.95 M_{\odot}$ do not evolve past the core-hydrogen burning stage in Hubble time.

Formation of compact binaries with WDs. A flowchart schematically presenting the typical scenario for formation of low-mass compact binaries with white-dwarf components and some endpoints of evolution is shown in Figure 5. Of course, not all possible scenarios are plotted, but only the most probable routes to SNe Ia and systems that may emit potentially detectable gravitational waves. For simplicity, we consider only the most general case when the first RLOF results in the formation of a common envelope.

The overwhelming majority of stars overflow their Roche lobes when they have He- or COcores. In stars with a mass below $(2.0-2.5) M_{\odot}$, helium cores are degenerate and if these stars overflow the Roche lobe prior to He-ignition, they produce helium white dwarfs. Binaries with non-degenerate He-core donors $\left(M \gtrsim(2.0-2.5) M_{\odot}\right)$ first form a He-star + MS-star pair that may be observed as a subdwarf (sdB or sdO) star with MS companion. When the He-star completes its evolution, a pair harbouring a CO white dwarf and a MS-star appears.

If after the first common-envelope stage the orbital separation of the binary $a \simeq$ several $R_{\odot}$ and the WD has a low-mass $\left(\lesssim 1.5 M_{\odot}\right)$ MS companion the pair may evolve into contact during the hydrogen-burning stage or shortly after because of loss of angular momentum by a magnetically coupled stellar wind and/or GW radiation. If, additionally, the mass-ratio of components is favourable for stable mass transfer a cataclysmic variable may form. If the WD belongs to the CO-variety and accreted hydrogen burns at the surface of the WD stably, the WD may accumulate enough mass to explode as a type Ia supernova; the same may happen if in the recurrent outbursts less mass is ejected than accreted (the so-called SD scenario for SNe Ia originally suggested by Whelan and Iben [451]; see, e.g., [217, 470, 126, 132, 102, 133, 215] and references therein for later studies).

Some CV systems that burn hydrogen stably or are in the stage of residual hydrogen burning after an outburst may be also observed as supersoft X-ray sources (see, e.g., [426, 339, 175, 471, $177,176])$.

If the WD belongs to the ONe-variety, it may experience an AIC into a neutron star due to electron captures on $\mathrm{Ne}$ and $\mathrm{Mg}$, and a low-mass X-ray binary may be formed.

The outcome of the evolution of a CV is not completely clear. It was hypothesized that the donor may be disrupted when its mass decreases below several hundredth of $M_{\odot}$ [358]. Note, that for $q \lesssim 0.02$, that may be attained in Hubble time, and the conventional picture of mass exchange may become non-valid, since the circularization radius becomes greater than the outer radius of the disk. Matter flowing in from the companion circularises onto unstable orbits. At $q \approx 0.02$, matter is added at $R_{\text {circ }}$ onto orbits that can become eccentric due to the $3: 1$ resonance. At $q \approx 0.005$ the

Living Reviews in Relativity

http://www . livingreviews . org/Irr-2006-6 


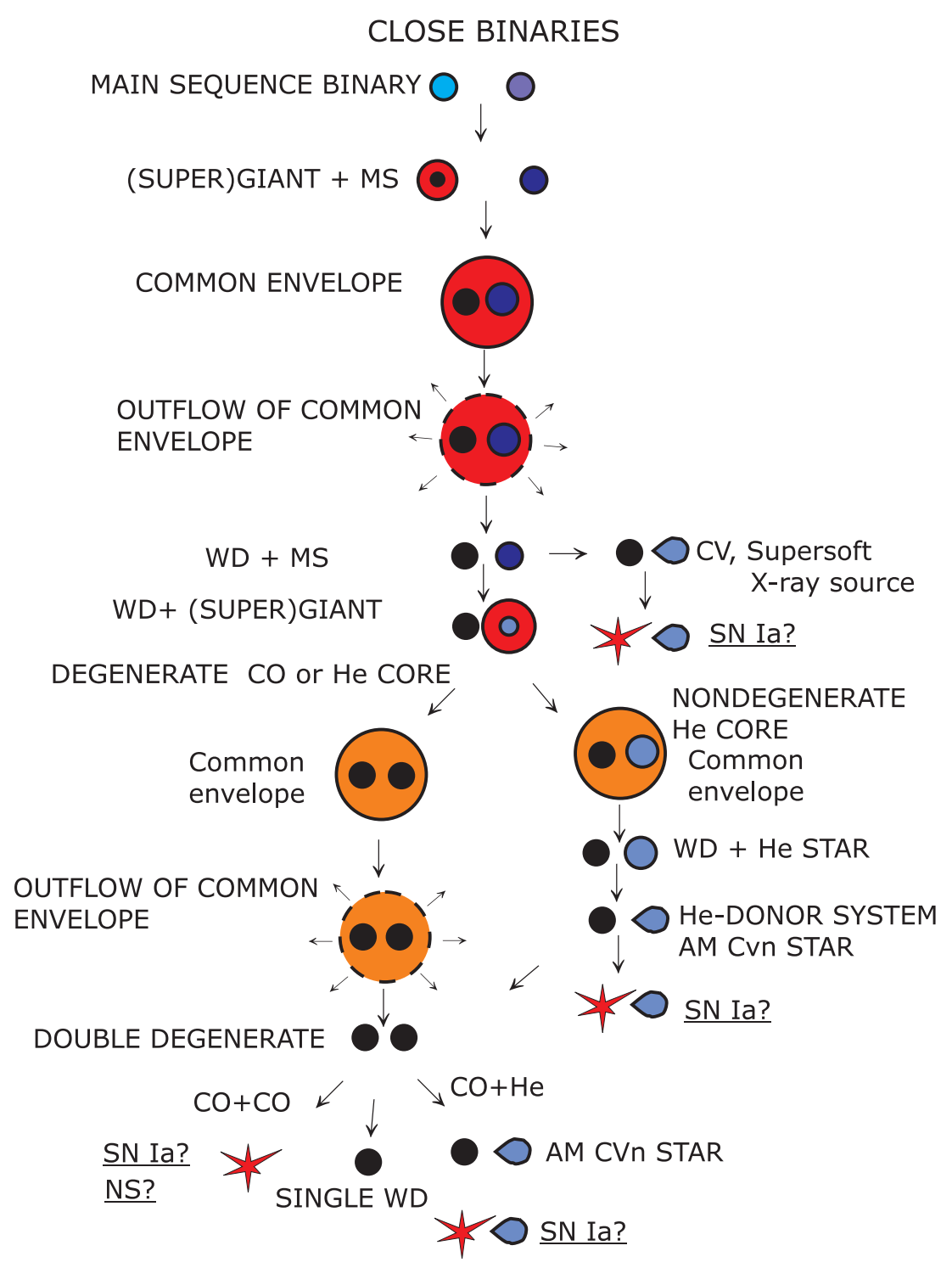

Figure 5: Formation of close binary dwarfs and their descendants (scale and colour-coding are arbitrary). 
circularization radius approaches the 2:1 Lindblad resonance. This can efficiently prevent mass being transferred onto the compact object. These endpoints of the evolution of binaries with low-mass donors, were, in fact, never studied.

The second common envelope may form when the companion to the WD overfills its Roche lobe. If the system avoids merger and the donor had a degenerate core, a close binary WD (or double-degenerate, DD) is formed. The fate of the DD is solely defined by GWR. The closest of them may be brought into contact by AML via GWR. The outcome of the contact depends on the chemical composition of the stars and their masses. The lighter of the two stars fills the Roche lobe first (by virtue of the mass-radius relation $R \propto M^{-1 / 3}$ ). For a zero-temperature WD the condition of stable mass transfer is $q<2 / 3$ (but see the more detailed discussion in Section 9). The merger of the CO-WD pair with a total mass exceeding $M_{\mathrm{Ch}}$ may result in a SN Ia leaving no remnant ("double-degenerate SN Ia scenario" first suggested by Webbink [443] and independently by Tutukov and Yungelson [418]) or in an AIC with formation of a single neutron star [259]. The issue of the merger outcome for $M_{\text {tot }}>M_{\mathrm{Ch}}$ still remains an unsolved issue and a topic of fierce discussion, see below. For total masses lower than $M_{\mathrm{Ch}}$ the formation of a single WD is expected.

If in a $\mathrm{CO}+\mathrm{He}$ WD dwarfs pair the conditions for stable mass exchange are fulfilled, an AM CVn system forms (see for details [417, 422, 280, 249, 120]).

\section{AGE OF HeWD+HeWD MERGERS}

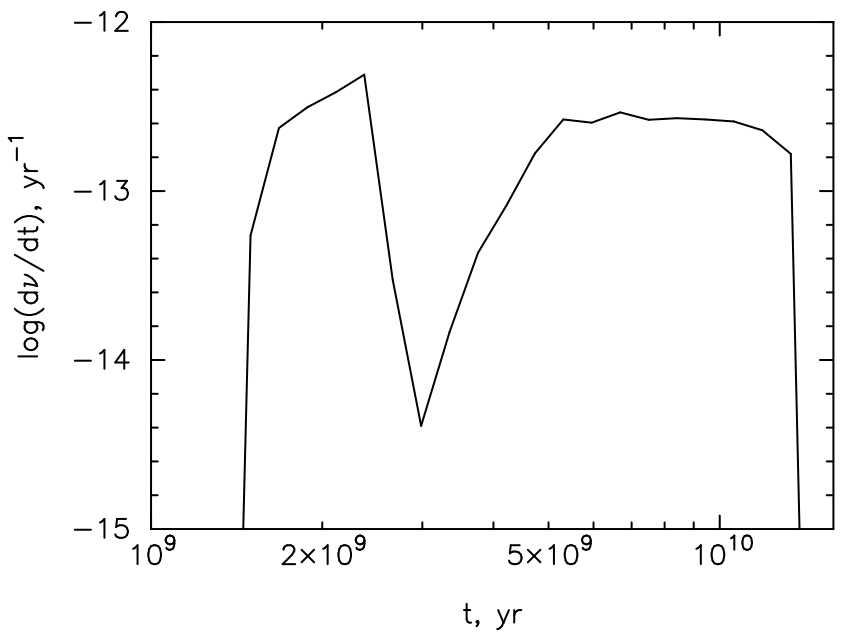

Figure 6: The age of merging pairs of helium WDs. Two components of the distribution correspond to the systems that experienced in the course of formation two or one common envelope episodes, respectively.

The current Galactic merger rate of close binary WDs is about $50 \%$ of their current birth rate $[285,423]$. It is not yet clear how the merger proceeds; it is possible that for $\mathrm{He}+\mathrm{He}$ or $\mathrm{CO}+\mathrm{He}$ pairs a helium star is an intermediate stage (see, e.g., [124]). It is important in this respect that binary white dwarfs at birth have a wide range of separations and merger of them may occur gigayears after formation. Formation of helium stars via merger may be at least partially responsible for the ultraviolet flux from the giant elliptical galaxies, where all hot stars finished their evolution long ago. This is illustrated by Figure 6 which shows the occurrence rate of mergers of pairs of He-WDs vs. age.

If the donor has a nondegenerate He-core $\left(M \gtrsim(2.0-2.5) M_{\odot}\right)$ and the system does not merge, after the second CE-stage a helium subdwarf + WD system may emerge. If the separation of components is sufficiently small, AML via GWR may bring the He-star into contact while He 


\begin{tabular}{l|ccccc}
\hline \hline Donor & CO-WD & MS/SG & He-star & He-WD & RG \\
\hline Counterpart & Close binary WD & Supersoft XRS & Blue sd & AM CVn & Symbiotic star \\
Mass transfer mode & Merger & RLOF & RLOF & RLOF & Wind \\
Young population & $\mathbf{1 0}^{-\mathbf{3}}$ & $10^{-4}$ & $10^{-4}$ & $10^{-5}$ & $10^{-6}$ \\
Old population & $\mathbf{1 0}^{-\mathbf{3}}$ & - & - & $10^{-5}$ & $10^{-6}$ \\
\hline \hline
\end{tabular}

Table 5: Occurrence rates of SNe Ia in the candidate progenitor systems (in $\mathrm{yr}^{-1}$ ), after [468]. SG stands for sub-giant, $R G$ for red giant, and XRS for X-ray source.

is still burning in its core. If $M_{\mathrm{He}} / M_{\mathrm{wd}} \lesssim 1.2$, stable mass exchange is possible with a typical $\dot{M} \sim 10^{-8} M_{\odot} \mathrm{yr}^{-1}$ [369]. Mass loss quenches nuclear burning and the helium star becomes "semidegenerate". An AM CVn-type system may be formed in this way (the "nondegenerate Hecore" branch of evolution in Figure 5). One cannot exclude that a Chandrasekhar mass may be accumulated by the WD in this channel of evolution, but the probability of such a scenario seems to be very low, $\sim 1 \%$ of the inferred Galactic rate of SNe Ia [382] $]^{11}$. If the He-star completes core He-burning before RLOF, it becomes a CO-WD. In Figure 5 it "jumps" into the "double degenerate" branch of evolution.

Type Ia supernovae. Table 5 summarizes order of magnitude model estimates of the occurrence rate of SNe Ia produced via different channels. For comparison, the rate of SNe Ia from wide binaries (symbiotic stars) is also given. The estimates are obtained by a population synthesis code used before in, e.g., [421, 470, 423] for the value of common envelope parameter $\alpha_{\text {ce }}=1$. The differences in the assumptions with other population synthesis codes or in the assumed parameters of the models result in numbers that vary by a factor of several; this is the reason for giving only order of magnitude estimates. The estimates are shown for $T=10$ Gyr after the beginning of star formation.

A "young" population had a constant star formation rate for $10 \mathrm{Gyr}$; in the "old" one the same amount of gas was converted into stars in $1 \mathrm{Gyr}$. Both populations have a mass comparable to the mass of the Galactic disk. We also list in the table the types of observed systems associated with a certain channel and the mode of mass transfer. These numbers have to be compared to the inferred Galactic occurrence rate of SNe Ia: $(4 \pm 2) \times 10^{-3} \mathrm{yr}^{-1}$ [53]. Table 5 shows that, say, for elliptical galaxies where star formation occurred in a burst, the DD scenario is the only one able to respond to the occurrence of SNe Ia, while in giant disk galaxies with continuing star formation other scenarios may contribute as well.

For about two decades since the prediction of the possibility of the merger of pairs of white dwarfs with total mass $\geq M_{\mathrm{Ch}}$, the apparent absence of observed DDs with proper mass and merger times shorter than Hubble time was considered as the major "observational" difficulty for the DD scenario. Theoretical models predicted that it may be necessary to investigate for binarity up to 1,000 field WDs with $V \lesssim 16-17$ for finding a proper candidate [287]. Currently, it is likely that this problem is resolved (see Section 8).

The merger of pairs of WDs occurs via an intermediate stage in which the lighter of the two dwarfs transforms into a disc $[417,28,259,235]$ from which the matter accretes onto the central object. It was shown for one-dimensional non-rotating models that the central C-ignition and SN Ia explosion are possible only for $\dot{M}_{\mathrm{a}} \lesssim(0.1-0.2) \dot{M}_{\text {Edd }}[291,402]$. But it was expected that in the merger products of binary dwarfs $\ddot{M}_{\mathrm{a}}$ is close to $\dot{M}_{\mathrm{Edd}} \sim 10^{-5} M_{\odot} \mathrm{yr}^{-1}$ [259] because of high viscosity in the transition layer between the core and the disk. For such an $\dot{M}_{\mathrm{a}}$, the nuclear burning will start at the core edge, propagate inward and convert the dwarf into an ONeMg one. The latter will collapse without a SN Ia [167]. However, an analysis of the role of deposition

${ }^{11}$ The recent discovery of SN Ia SN 2003fg with a mass estimate $\sim 2 M_{\odot}[152,172]$ may support this scenario. 
of angular momentum into a central object by Piersanti and coauthors [315, 316] led them to conclusion that, as a result of the spin-up of rotation of the WD, instabilities associated with rotation, deformation of the WD, and AML by a distorted configuration via GWR, an $\dot{M}_{\text {a }}$ that is initially $\sim 10^{-5} M_{\odot} \mathrm{yr}^{-1}$ decreases to $\simeq 4 \times 10^{-7} M_{\odot} \mathrm{yr}^{-1}$. For this $\dot{M}_{\text {a }}$ close-to-center ignition of carbon becomes possible. The efficiency of the mechanism suggested in [315, 316] is disputed, for instance, by Saio and Nomoto [364] who found that an off-center carbon ignition occurs even when the effect of stellar rotation is included, if $\dot{M}_{\mathrm{a}}>3 \times 10^{-6} M_{\odot} \mathrm{yr}^{-1}$. The latter authors find that the critical accretion rate for the off-center ignition is hardly changed by the effect of rotation. The problem has to be considered as unsettled until a better understanding of redistribution of angular momentum during the merger process will become available.

Because of a long absence of apparent candidates for the DD scenario and its theoretical problems, the SD scenario is often considered as the most promising one. However, it also encounters severe problems. Even stably burning white dwarfs must have radiatively driven winds. At $\dot{M}_{\text {accr }} \lesssim 10^{-8} M_{\odot} \mathrm{yr}^{-1}$ all accumulated mass is lost in nova explosions [331, 461]. Even if $\dot{M}_{\text {accr }}$ allows accumulation of a He-layer, most of the latter is lost after the He-flash [163, 54, 314], dynamically or via the frictional interaction of the binary components with the giant-size common envelope. As a result, mass accumulation efficiency is always $<1$ and may be even negative. On the other hand, it was noted that the flashes become less violent and more effective accumulation of matter may occur if mass is transferred on a rate close to the thermal one or the dwarf is rapidly rotating [160, 470, 171, 132, 102, 465, 464]. Thus, crucial for this SN Ia scenario are the range of donor masses that may support mass-loss rates "efficient" for the growth of WD, mechanisms for stabilizing mass loss in the necessary range, convection and angular momentum transfer in the accreted layer that define the amount of mass loss in the outbursts, and the amount of matter that escape in the wind ${ }^{12}$. If the diversity of SNe Ia is associated with the spread of mass of the exploding objects, it would be more easily explained in a SD scenario, since the latter allows white dwarfs to grow efficiently in mass by shell burning, which is stabilized by accretion-induced spin-up. This inference may be supported by the discovery of the "super-Chandrasekhar" mass SN Ia SN $2003 \mathrm{fg}$ (mass estimate $\sim 2 M_{\odot}[152,172]$ ). Even under assumption of the most favourable conditions for a SN Ia in the SD scenario, the estimates of the current Galactic occurrence rate for this channel do not exceed $1 \times 10^{-3} \mathrm{yr}^{-1}$ [132], i.e. they may contribute up to $50 \%$ of the lowest estimate of the inferred Galactic SN Ia occurrence rate.

On the observational side, the major objection to the SD scenario comes from the fact that no hydrogen is observed in the spectra of SNe Ia, while it is expected that $\sim 0.15 M_{\odot}$ of $\mathrm{H}$-rich matter may be stripped from the companion by the SN shell [246] ${ }^{13}$. Hydrogen may be discovered both in very early and late optical spectra of SNe and in radio- and X-ray ranges [87, 246, 214]. Panagia et al. [304] find a firm upper limit to a steady mass-loss rate for individual SN systems of $\sim 3 \times 10^{-8} M_{\odot} \mathrm{yr}^{-1}$. As well, no expected [246, 52] high luminosity and/or high velocity former companions to exploding WD were discovered as yet ${ }^{14}$. The SD scenario also predicts the existence of many more supersoft X-ray sources than are expected from observations, even considering severe problems in estimating incompleteness of the samples of the latter (see for instance [82]).

To summarize, the problem of progenitors of SNe Ia is still unsettled. Large uncertainties in the model parameters involved in the computation of the evolution leading to a SN Ia and in computations of the explosions themselves, do not allow to exclude any type of progenitors. The existence of at least two families of progenitors is suggested by observations (see, e.g., [245]). A high proportion of "peculiar" SN Ia of $(36 \pm 9) \%$ [216] suggests a large spread in the ignition conditions in the exploding objects that also may be attributed to the diversity of progenitors.

\footnotetext{
${ }^{12}$ All these caveats date back as far as to MacDonalds' 1984 paper [242] but are still not resolved.

${ }^{13}$ The recently discovered hydrogen-rich SN Ia 2001ic and similarly 1997cy [130] may belong to the so-called SN 1.5 type or occur in a symbiotic system [57].

${ }^{14}$ The reported discovery of Tycho Brahe's 1572 SN Ia companion [360] is not confirmed as yet.
} 
Note that a high proportion of "peculiar" SNe Ia casts a certain doubt to their use as standard candles for cosmology.

As shown in the flowchart in Figure 5, there are configurations for which it is expected that stable accretion of $\mathrm{He}$ onto a CO-WD occurs: in AM CVn systems in the double-degenerate formation channel and in precursors of AM CVn systems in the helium-star channel. In the latter systems the mass exchange rate is close to $(1-3) \times 10^{-8} M_{\odot} \mathrm{yr}^{-1}$, practically irrespective of the combination of donor and accretor mass. It was suggested that in such systems the accumulation of a $\sim 0.1 M_{\odot}$ degenerate He-layer onto a $(0.6-0.9) M_{\odot}$ accretor is possible prior to He-detonation and that the latter initiates a compressional wave that results in the central detonation of carbon [230, 232, 459, 231]; even if central carbon ignition does not occur, the scale of the event is comparable to weak SNe $[218,162,414]$. For a certain time these events involving sub-Chandrasekhar mass accretors (nicknamed "edge-lit detonations", ELD) that may occur at the rates of $\sim 10^{-3} \mathrm{yr}^{-1}$ were considered as one of the alternative mechanisms for SNe Ia, although it was shown by Höfflich and Khokhlov [148] that the behaviour of light-curves produced by them does not resemble any of the known SNe Ia. Thus, until recently, the real identification of these events remained a problem. However, it was shown recently by Yoon and Langer [463], who considered angular-momentum accretion effects, that the helium envelope is heated efficiently by friction in the differentially rotating spun-up layers. As a result, helium ignites much earlier and under much less degenerate conditions compared to the corresponding non-rotating case. If the efficiency of energy dissipation is high enough, detonation may be avoided and, instead of a $\mathrm{SN}$, recurrent helium novae may occur. The outburst, typically, happens after accumulation of $0.02 M_{\odot}$. Currently, there is one object known, identified as He-nova - V445 Pup [439, 440, 9, 10]. If He-novae are really associated with mass-transfer from low-mass helium stars to $\mathrm{CO}$ white dwarfs, then the estimates of the birth rate of the latter systems $\left(\approx 0.6 \times 10^{-3} \mathrm{yr}^{-1}\right)$ [473] and of the amount of matter available for transfer $\left(\approx 0.2 M_{\odot}\right)$ give an occurrence rate of He-novae of $\sim 0.1 \mathrm{yr}^{-1}$, i.e. one He-nova per several 100 "ordinary" hydrogen-rich novae.

As we mentioned above, intermediate mass donors, before becoming white dwarfs, pass through the stage of a helium star. If the mass of the latter is above $\simeq 0.8 M_{\odot}$, it expands to giant dimensions after exhaustion of He in the core and may overflow the Roche lobe and, under proper conditions, transfer mass stably [161]. For the range of mass-accretion rates expected for these stars, both the conditions for stable and unstable helium burning may be fulfilled. In the former case the accumulation of $M_{\mathrm{Ch}}$ and a SN Ia become possible, as it was shown explicitly by Yoon and Langer [462]. However, the probability of such a SN Ia is only $\sim 10^{-5} \mathrm{yr}^{-1}$.

Ultra-compact X-ray binaries. The suggested channels for formation of UCXBs in the field are, in fact, "hybrids" of scenarios presented in Figures 4 and 5. In progenitors of these systems, the primary becomes a neutron star, while the secondary is not massive enough. Then, several scenarios similar to the scenarios for the systems with the first-formed white dwarf are open. A white dwarf may overflow the Roche lobe due to systemic AML via GWR. A low-mass companion to a neutron star may overflow the Roche lobe at the end of the main sequence and become a lowmass He-rich donor. A core helium-burning star may be brought in contact by AML due to GWR; mass loss quenches nuclear burning and the donor becomes a helium "semidegenerate" object. An additional scenario is provided by the formation of a neutron-star component by AIC of an accreting white dwarf. We refer the reader to the pioneering papers [411, 288, 394, 369, 412, 101, 434] and to more recent studies [285, 472, 321, 311, 25, 166, 429, 430, 191, 181]. An analysis of the chemical composition of donors in these systems seems to be a promising way for discrimination between systems of different origin $[278,279,166,448]$ : Helium dwarf donors should display products of H-burning, while He-star descendants should display products of He-burning products. Actually, both carbon/oxygen and helium/nitrogen discs in UCXBs are discovered [277].

In globular clusters, UCXBs are formed most probably by dynamical interactions, as first suggested by Fabian et al. [93] (see, e.g., [342, 170, 432, 233, 26] and references therein for the 
latest studies on the topic). 


\section{Observations}

The state of interrelations between observations and theoretical interpretations are different for different groups of compact binaries. Such cataclysmic variables as novae stars have been observed for centuries, their lower-amplitude cousins (including AM CVn-type stars) for decades, and their origin and evolution found their theoretical explanation after the role of common envelopes and gravitational waves radiation and magnetic braking were recognized [300, 301, 417, 435]. At present, about 2,000 CVs are known; see the online catalogue by Downes et al. [84] at [383].

More than 600 of them have measured orbital periods; see the online catalogue by Kolb and Ritter [350] at [351].

In particular, there are at present 17 confirmed AM CVn-stars with measured or estimated periods and two more candidate systems; see the lists and references in [272,390] and [275].

Ultracompact X-ray binaries were discovered with the advent of the X-ray astronomy era in the late 1960s and may be found in the first published catalogues of X-ray sources (see for instance [118]). Their detailed optical investigation became possible only with $8 \mathrm{~m}$-class telescopes. Currently, 12 UCXB systems with measured or suspected periods are known plus six candidates; six of the known systems reside in globular clusters (see lists and references in [277, 20]). Contrary to cataclysmic binaries, the place of UCXBs in the scenarios of evolution of close binaries, their origin and evolution were studied already before optical identification [418, 340, 411].

Unlike CVs and UCXBs, the existence of close detached white dwarfs (DDs) was first deduced from the analysis of scenarios for the evolution of close binaries [443, 417, 418, 160, 444]. Since it was also inferred that DDs may be precursors of SNe Ia, this theoretical prediction stimulated optical surveys for DDs and the first of them was detected in 1988 by Saffer, Liebert, and Olszewski [362]. However, a series of surveys for DDs performed over a decade [352, 41, 108, 248, 363] resulted in only about a dozen of definite detections [247].

\subsection{SPY project}

The major effort to discover DDs was undertaken by the "ESO Supernovae Ia Progenitors surveY (SPY)" project: a systematic radial velocity survey for DDs with the UVES spectrograph at the ESO VLT (with PI R. Napiwotzky; see [265, 266, 372, 268, 267] for the details of the project and [267] for the latest published results). The project was aimed at DDs as potential progenitors of type Ia supernovae, but brought as a by-product an immense wealth of data on white dwarfs. More than 1,000 white dwarfs and pre-white dwarfs were observed (practically all white dwarfs brighter than $V \approx 16.5$ available for observations from the ESO site in Chile). SPY tremendously increased the number of detected DDs to more than 150 . Their system parameters are continuously determined from follow-up observations. Figure 7 shows the total masses of the currently known close DDs vs. orbital periods and compares them with the Chandrasekhar mass and the critical periods necessary for merger of components in Hubble time for given $M_{\text {tot }}$ (data available in the fall of 2005; R. Napiwotzky, private communication) Altogether, $\sim 5$ super-Chandrasekhar total mass DDs are expected to be found by SPY. At the moment, several systems with masses close to the Chandrasekhar limit and merger time shorter than Hubble time, including a probable SN Ia progenitor candidate are already detected. The second candidate super-Chandrasekhar mass binary white dwarf was discovered by Tovmassian et al. [404].

Figure 8 shows the position of the observed components of known DDs vs. the theoretical expectations with account for observational selection effects in the $P_{\text {orb }}-m$ diagram [287]. The agreement may be considered as quite satisfactory. 


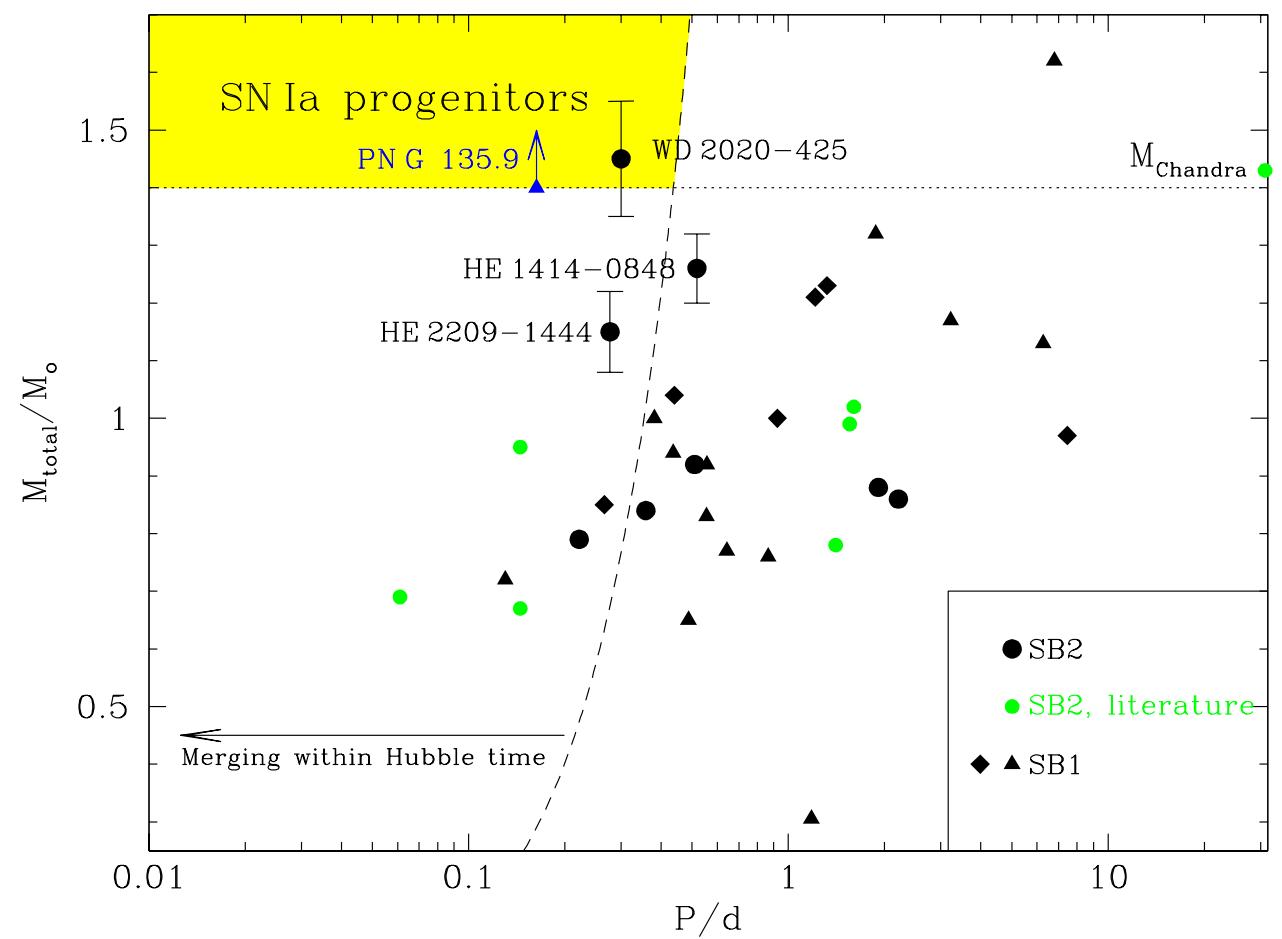

Figure 7: Known close binaries with two WD components, or a WD and a sd component. Green circles mark systems known prior to the SPY project. Black filled symbols mark the positions of $D D s$ and $W D+$ sd systems detected in the SPY project. A blue triangle marks the positions of the WD component of the binary planetary nebula nucleus PN G135.9+55.91 detected by Tovmassian et al. [404]. (Courtesy R. Napiwotzki.)

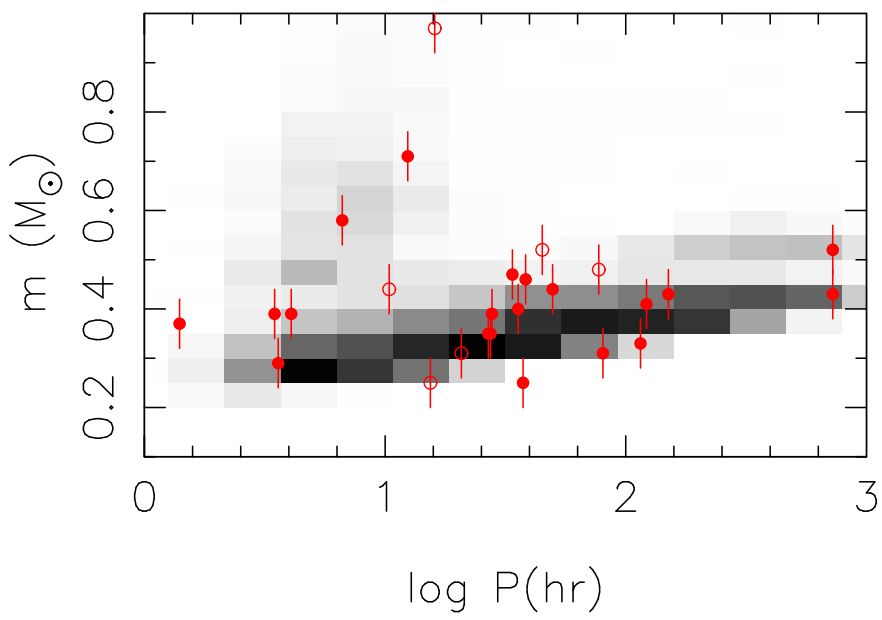

Figure 8: The position of the primary components of known DDs in the "orbital-period-mass" diagram. The underlying gray scale plot is a model prediction from Nelemans et al. [285]. (Figure from [276].)

Living Reviews in Relativity

http://www. livingreviews.org/lrr-2006-6 


\section{Evolution of Interacting Double-Degenerate Systems}

Angular momentum losses via GWR may bring detached double degenerates into contact. The mass-radius relation for degenerate stars has a negative power $(\simeq-1 / 3$ for WDs with a mass exceeding $0.1 M_{\odot}$, irrespective of their chemical composition and temperature [75]). Hence, the lower mass WD fills its Roche lobe first.

In a binary with stable mass transfer the change of the radius of the donor exactly matches the change of its Roche lobe. This condition combined with an approximation to the size of the Roche lobe valid for low $q[194]$,

$$
R_{\mathrm{L}} \approx 0.4622 a\left(\frac{m}{M+m}\right)^{1 / 3}
$$

where $m$ and $M$ are, respectively, the masses of the prospective donor and accretor, renders two relations important for the study of compact binaries.

1. It provides a relation between the orbital period of the binary $P_{\text {orb }}$ and the mass $M_{2}$ and radius $R_{2}$ of the donor:

$$
P_{\text {orb }} \simeq 101 \mathrm{~s}\left(\frac{R_{2}}{0.01 R_{\odot}}\right)^{3 / 2}\left(\frac{0.1 M_{\odot}}{M_{2}}\right)^{1 / 2}
$$

2. It allows to derive the rate of mass transfer for a semidetached binary in which mass transfer is driven by angular momentum losses:

$$
\frac{\dot{m}}{m}=\left(\frac{\dot{J}}{J}\right)_{\mathrm{GWR}} \times\left(\frac{\zeta(m)}{2}+\frac{5}{6}-\frac{m}{M}\right)^{-1}
$$

where $\zeta(m)=d \ln r / d \ln m$.

For the mass transfer to be stable, the term in the brackets must be positive, i.e.

$$
\frac{m}{M}<\frac{5}{6}+\frac{\zeta(m)}{2}
$$

Violation of this criterion results in mass loss by the donor on a dynamical time scale and, most probably, merger of components. Of course, Equation (65) clearly oversimplifies the conditions for stable mass exchange. A rigorous treatment has to include a consideration of tidal effects, angular momentum exchange, and possible super-Eddington $\dot{M}$ immediately after RLOF by the donor and associated common-envelope formation, possible ignition of accreted helium [446, 136, 250, 120]. However, the study of these effects is still in the embryonic state and usually the evolution of IDDs or other binaries with WD donors is calculated applying $M-R$ relations, without considering detailed models of WDs (or low-mass helium stars in the appropriate formation channel); see, e.g., [300, 436, 99, 417, 340, 395, 433, 422, 285, 472, 286]. Figure 9 shows examples of the evolution for systems with a helium degenerate donor or a low-mass "semidegenerate" helium star donor and a carbon-oxygen accretor with initial masses that are currently thought to be typical for progenitors of AM CVn systems -0.2 and $0.6 M_{\odot}$, respectively. For Figure 9 the mass-radius relation for zerotemperature white dwarfs from the article by Verbunt and Rappaport [433] is used; for the low-mass He-star mass-radius relation approximating results of evolutionary computations by Tutukov and Fedorova [409] were used: $R \approx 0.043 m^{-0.062}$ (in solar units). The same equations are applied for obtaining the model of the population of AM CVn-stars discussed in Sections 10, 11, and 12. The mass of the donor in the system may be a discriminator between the formation channels. 


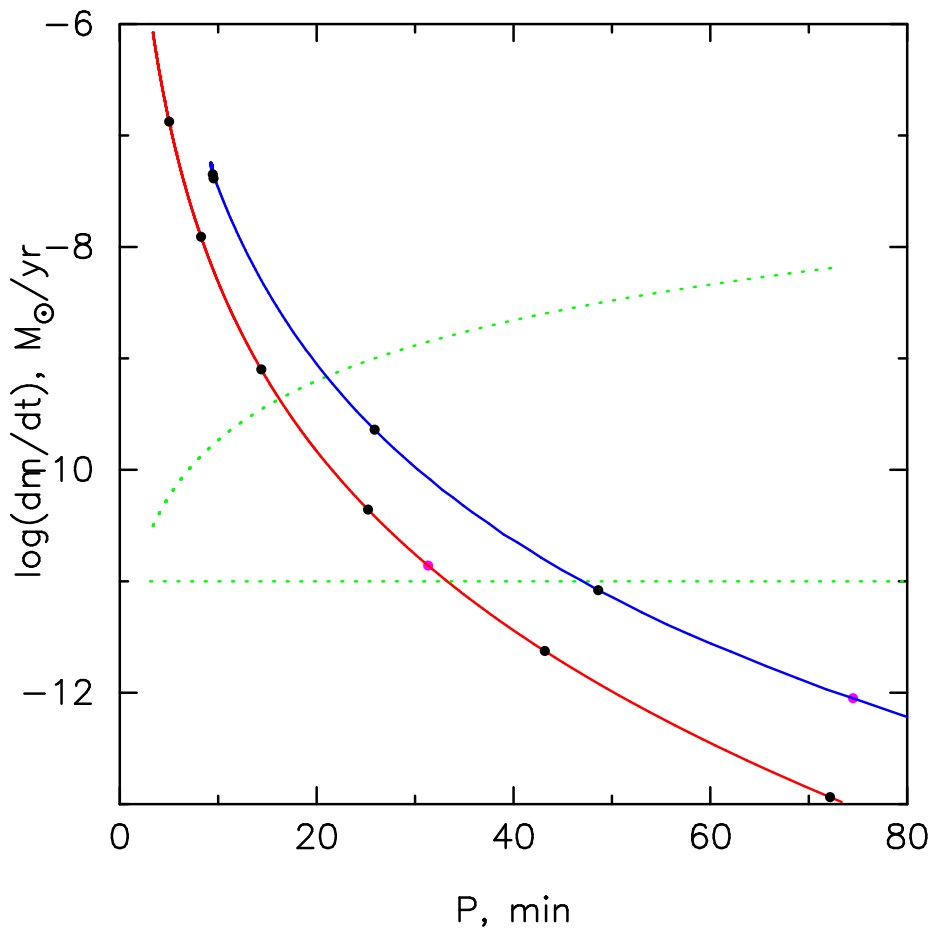

Figure 9: Mass-loss rate vs. orbital period for "typical" AM CVn-stars: an interacting double degenerate system with initial masses of donor and accretor $0.2 M_{\odot}$ and $0.6 M_{\odot}$ (red line) and a "semidegenerate" low-mass helium star donor plus white dwarf accretor of the same initial masses (blue line). Black dots on the red curve mark positions of the system at $\log T(\mathrm{yr})=5,6,7,8,9,10$ from Roche-lobe overflow; on the blue curve they mark $\log T(\mathrm{yr})=5,6,7,8$. Green lines mark lower and upper limits of the disk instability region according to Tsugawa and Osaki [406]. Below the magenta circles $q<0.02$ and conventional evolutionary computations may be not adequate for description of mass-transfer process (see the text).

Living Reviews in Relativity

http://www. livingreviews . org/lrr-2006-6 
For instance, a large mass of the donor $\left(0.18 M_{\odot}\right)$ found for the prototype of the class, AM CVn itself [354] favours the helium channel for this system.

From the Equations $(22,62,64)$ it follows that for $m \ll M$ the mass loss rate scales as $M^{1 / 3}$. As a result, for all combinations of donor and accretor, the $P-\dot{m}$ lines form two rather narrow strips within which they converge with decreasing $m$. We should note that the time span between formation of a pair of WDs and contact may be from several Myr to several Gyr [422]. This means that the approximation of zero-temperature white dwarfs is not always valid. Below we discuss the implications of finite entropy of the donors for the population of AM CVn-stars.

The "theoretical" model of evolution from shorter periods to longer ones is supported by observations which found that the UV luminosity of AM CVn-stars is increasing as the orbital period gets shorter, since shorter periods are associated with higher $\dot{M}$ [337].

Note that there is a peculiar difference between white dwarf pairs that merge and pairs that start stable mass exchange. The pairs that coalesce stop emitting GWs in a relatively small timescale (of the order of the period of the last stable orbit, typically a few minutes) [235]. Thus, if we would be lucky to observe a chirping WD and a sudden disappearance of the signal, this will manifest a merger. However, the chance of such event is small since the Galactic occurrence rate of mergers of WDsis $\sim 10^{-2} \mathrm{yr}^{-1}$ only.

Apart from "double-degenerate" and "helium-star" channels for the formation of AM CVnstars, there exists the third, "CV"-channel [411, 412, 318]. In this channel, the donor star fills its Roche lobe at the main-sequence stage or just after its completion. For such donors the chemical inhomogeneity inhibits complete mixing at $M \simeq 0.3 M_{\odot}$ typical for initially non-evolved donors. The mixing is delayed to lower masses and as a result the donors become helium dwarfs with some traces of hydrogen. After reaching the minimum period they start to evolve to the longer ones. The minimum of periods for these systems is $\simeq 5-7$ min. However, the birth rate of systems that can penetrate the region occupied by observed AM CVn-stars is much lower than the birth rate in "double degenerate" and "helium-star" channels and we do not take this channel into account below. 


\section{Gravitational Waves from Compact Binaries with White- Dwarf Components}

It was expected initially that contact W UMa binaries will dominate the gravitational wave spectrum at low frequencies [256]. However, it was shown in [417, 252, 160, 92, 222] that it is, most probably, totally dominated by detached and semidetached double white dwarfs.

As soon as it was recognized that the birth rate of Galactic close double white dwarfs may be rather high and even before the first close DD was detected, Evans, Iben, and Smarr in 1987 [92] accomplished an analytical study of the detectability of the signal from the Galactic ensemble of DDs, assuming certain average parameters for DDs. Their main findings may be formulated as follows. Let us assume that there exists a certain distribution of DDs over frequency of the signal $f$ and strain amplitude $h: n(f, h)$. The weakest signal is $h_{\mathrm{w}}$. For the time span of observations $\tau_{\text {int }}$, the elementary resolution bin of the detector is $\Delta f_{\text {int }} \approx 1 / \tau_{\text {int }}$. Then, integration of $n(f, h)$ over amplitude down to a certain limiting $h$ and over $\Delta f$ gives the mean number of sources per unit resolution bin for a volume defined by $h$. If for a certain $h_{n}$

$$
\int_{h_{n}}^{\infty}\left(\frac{d n}{d f d h}\right) \Delta \nu_{\mathrm{int}}=1,
$$

then all sources with $h_{n}>h>h_{\mathrm{w}}$ overlap. If in a certain resolution bin $h_{n}>h_{\mathrm{w}}$ does not exist, individual sources may be resolved in this bin for a given integration time (if they are above the detector's noise level). In the bins where binaries overlap they produce so-called "confusion noise": an incoherent sum of signals; the frequency, above which the resolution of individual sources becomes possible got the name of "confusion limit", $f_{\mathrm{c}}$. Evans et al. found $f_{\mathrm{c}} \approx 10 \mathrm{mHz}$ and $3 \mathrm{mHz}$ for integration times $10^{6} \mathrm{~s}$ and $10^{8} \mathrm{~s}$, respectively.

Independently, the effect of confusion of Galactic binaries was demonstrated by Lipunov, Postnov, and Prokhorov [223] who used simple analytical estimates of the GW confusion background produced by unresolved binaries whose evolution is driven by GWs only; in this approximation, the expected level of the background depends solely on the Galactic merger rate of binary WDs (see [122] for more details). Later, more involved analytic studies of the GW background produced by binary stars at low frequencies were continued in [96, 143, 329, 142].

A more detailed approach to the estimate of the GW background is possible using population synthesis models [222, 445, 285, 286].

Nelemans et al. [285] constructed a model of the gravitational wave signal from the Galactic disk population of binaries containing two compact objects. The model included detached DDs, semidetached DDs, detached systems of NSs and BHs with WD companions, binary NSs and BHs. For the details of the model we refer the reader to the original paper and references therein. Table 6 shows the number of systems with different combinations of components in the Nelemans et al. model $^{15}$. Note that these numbers strongly depend on the assumptions in the population synthesis code, especially on the normalization of stellar birth rate, star formation history, distributions of binaries over initial masses of components and their orbital separations, treatment of stellar evolution, common envelope formalism, etc. For binaries with relativistic components (i.e. descending from massive stars) an additional uncertainty is brought in by assumptions on stellar wind mass loss and natal kicks. The factor of uncertainty in the estimated number of systems of a specific type may be up to a factor $\sim 10$ (cf. [131, 285, 423, 155]). Thus these numbers have to be taken with some caution; we will show the effect of changing some of approximations below. Table 6 immediately shows that detached DDs, as expected, dominate the population of compact binaries.

Population synthesis computations yield the ensemble of Galactic binaries at a given epoch with their specific parameters $M_{1}, M_{2}$, and $a$. Figure 10 shows examples of the relation between

\footnotetext{
${ }^{15}$ These numbers, like also for models discussed below, represent one random realization of the model and are subject to Poisson noise.
}

Living Reviews in Relativity

http: //www . livingreviews . org/lrr-2006-6 


\begin{tabular}{l|ccc}
\hline \hline Type & Birth rate & Merger rate & Number \\
\hline Detached DD & $2.5 \times 10^{-2}$ & $1.1 \times 10^{-2}$ & $1.1 \times 10^{8}$ \\
Semidetached DD & $3.3 \times 10^{-3}$ & - & $4.2 \times 10^{7}$ \\
NS + WD & $2.4 \times 10^{-4}$ & $1.4 \times 10^{-4}$ & $2.2 \times 10^{6}$ \\
$\mathrm{NS}+\mathrm{NS}$ & $5.7 \times 10^{-5}$ & $2.4 \times 10^{-5}$ & $7.5 \times 10^{5}$ \\
$\mathrm{BH}+\mathrm{WD}$ & $8.2 \times 10^{-5}$ & $1.9 \times 10^{-6}$ & $1.4 \times 10^{6}$ \\
$\mathrm{BH}+\mathrm{NS}$ & $2.6 \times 10^{-5}$ & $2.9 \times 10^{-6}$ & $4.7 \times 10^{5}$ \\
$\mathrm{BH}+\mathrm{BH}$ & $1.6 \times 10^{-4}$ & - & $2.8 \times 10^{6}$ \\
\hline \hline
\end{tabular}

Table 6: Current birth rates and merger rates per year for Galactic disk binaries containing two compact objects and their total number in the Galactic disk [285].

frequency of emitted radiation and amplitude of the signals from a "typical" double degenerate system that evolves into contact and merges, for an initially detached double degenerate system that stably exchanges matter after contact, i.e. an AM CVn-type star and its progenitor, and for an UCXB and its progenitor. For the AM CVn system effective spin-orbital coupling is assumed [280, 249]. For the system with a NS, the mass exchange rate is limited by the Eddington one and excess of the matter is "re-ejected" from the system" (see Section 3.2.3 and [472]). Note that for an AM CVn-type star it takes only $\sim 300 \mathrm{Myr}$ after contact to evolve to $\log f=-3$ which explains their accumulation at lower $f$. For UCXBs this time span is only $\sim 20 \mathrm{Myr}$. In the discussed model, the systems are distributed randomly in the Galactic disk according to

$$
\rho(R, z)=\rho_{0} e^{-R / H} \operatorname{sech}(z / \beta)^{2} \mathrm{pc}^{-3},
$$

where $H=2.5 \mathrm{kpc}$ [361] and $\beta=200 \mathrm{pc}$. The Sun is located at $R_{\odot}=8.5 \mathrm{kpc}$ and $z_{\odot}=-30 \mathrm{pc}$.

Then it is possible to compute strain amplitude for each system. The power spectrum of the signal from the population of binaries as it would be detected by a gravitational wave detector, may be simulated by computation of the distribution of binaries over $\Delta f=1 / T$ wide bins, with $T$ being the total integration time. Figure 11 shows the resulting confusion limited background signal. In Figure 12 the number of systems per bin is plotted. The assumed integration time is $T=1$ yr. Semidetached double white dwarfs, which are less numerous than their detached cousins and have lower strain amplitude dominate the number of systems per bin in the frequency interval $-3.4 \lesssim \log f(\mathrm{~Hz}) \lesssim-3.0$ producing a peak there, as explained in the comment to Figure 10 .

Figure 11 shows that there are many systems with a signal amplitude much higher than the average in the bins with $f<f_{\mathrm{c}}$, suggesting that even in the frequency range seized by confusion noise some systems may be detectable above the noise.

Population synthesis also shows that the notion of a unique "confusion limit" is an artifact of the assumption of a continuous distribution of systems over their parameters. For a discrete population of sources it appears that for a given integration time there is a range of frequencies where there are both empty resolution bins and bins containing more than one system (see Figure 13). For this "statistical" notion of $f_{c}$, Nelemans et al. [285] get the first bin containing exactly one system at $\log f(\mathrm{~Hz}) \approx-2.84$, while up to $\log f(\mathrm{~Hz}) \approx-2.3$ there are bins containing more than one system.

As we noted above, predictions of the population synthesis models are sensitive to the assumptions of the model; one of the most important is the treatment of common envelopes (see Section 3.5). Figure 14 compares the average gravitational waves background formed by Galactic population of white dwarfs under different assumptions on star formation history, IMF, assumed Hubble time, and treatment of some details of stellar evolution (cf. [285, 286]). The comparison with the work of other authors $[141,445,374]$ shows that both the frequency of the confusion limit 


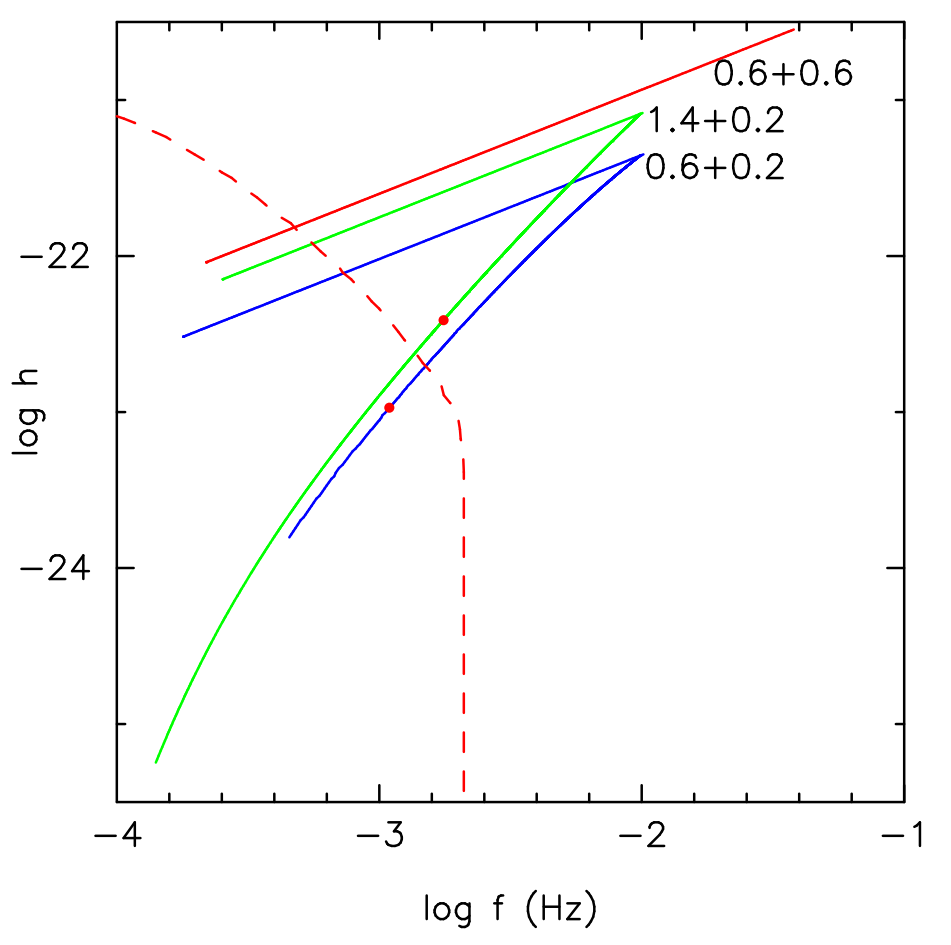

Figure 10: Dependence of the dimensionless strain amplitude for a WD $+W D$ detached system with initial masses of the components of $0.6 M_{\odot}+0.6 M_{\odot}$ (red line), a WD $+W D$ system with $0.6 M_{\odot}+0.2 M_{\odot}$ (blue line) and a WD $+N S$ system with $1.4 M_{\odot}+0.2 M_{\odot}$ (green line). All systems have an initial separation of components $1 R_{\odot}$ and are assumed to be at a distance of $1 \mathrm{kpc}$ (i.e. the actual strength of the signal has to be scaled with factor $1 / d$, with $d$ in $\mathrm{kpc})$. For the DD system the line shows an evolution into contact, while for the two other systems the upper branches show pre-contact evolution and lower branches - a post-contact evolution with mass exchange. The total time-span of evolution covered by the tracks is 13.5 Gyr. Red dots mark the positions of systems with mass-ratio of components $q=0.02$ below which the conventional picture of evolution with a mass exchange may be not valid. The red dashed line marks the position of the confusion limit as determined in [286]. 


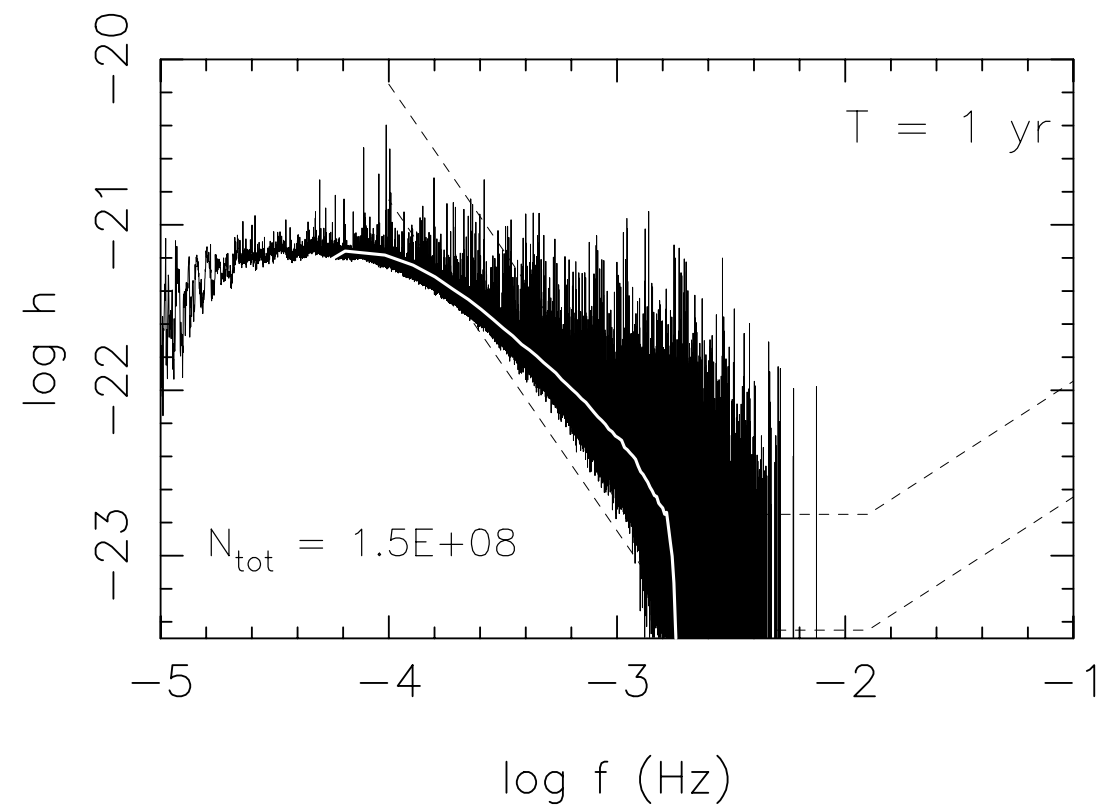

Figure 11: GWR background produced by detached and semidetached double white dwarfs as it would be detected at the Earth. The assumed integration time is $1 \mathrm{yr}$. The 'noisy' black line gives the total power spectrum, the white line the average. The dashed lines show the expected LISA sensitivity for a $S / N$ of 1 and 5 [210]. Semidetached double white dwarfs contribute to the peak between $\log f \simeq-3.4$ and -3.0 . (Figure from [285].)

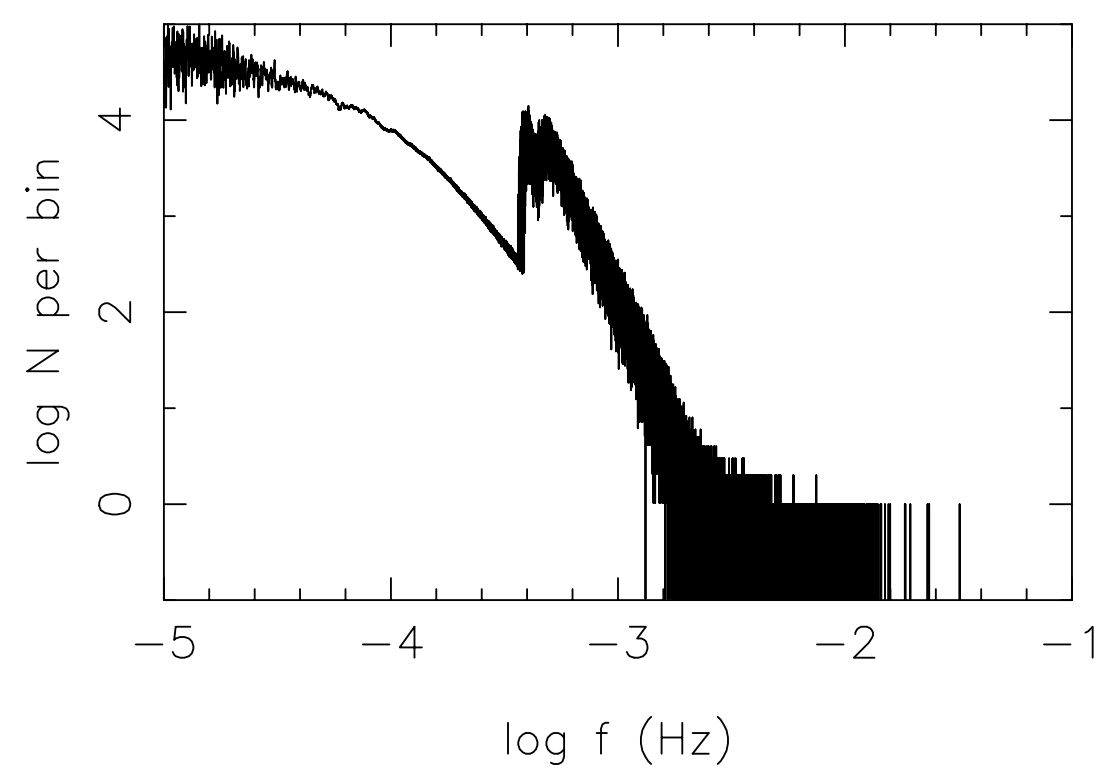

Figure 12: The number of systems per bin on a logarithmic scale. Semidetached double white dwarfs contribute to the peak between $\log f \simeq-3.4$ and -3.0 . (Figure from [285].) 


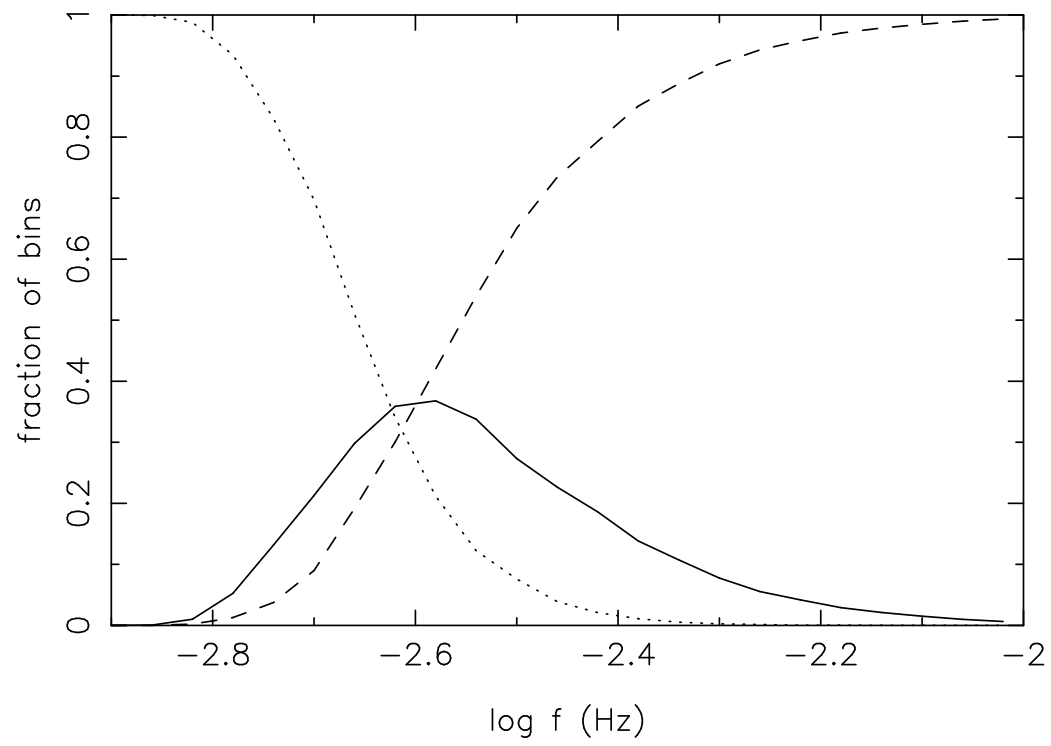

Figure 13: Fraction of bins that contain exactly one system (solid line), empty bins (dashed line), and bins that contain more than one system (dotted line) as function of the frequency of the signals. (Figure from [285].)

and the level of confusion noise are uncertain within a factor of $\sim 4$. This uncertainty is clearly high enough to influence seriously the estimates of the possibilities for a detection of compact binaries. Note, however, that there are systems expected to be detected above the noise in all models (see below).

Within the model of Nelemans et al. [285] there are about 12,100 detached DD systems that can be resolved above $f_{\mathrm{c}}$ and $\approx 6,100$ systems with $f<f_{\mathrm{c}}$ that are detectable above the noise. This result was confirmed in a follow-up paper [286] which used a more up-to-date SFH and Galaxy model (this resulted in a slight decrease of the number of "detectable" systems - to 11,000). The frequency - strain amplitude diagram for DD systems is plotted in the left panel of Figure 15. In the latter study the following was noted. Previous studies of GW emission of the AM CVn systems [142, 285] have found that they hardly contribute to the GW background noise, even despite at $f=(0.3-1.0) \mathrm{mHz}$ they outnumber the detached DDs. This happens because at these $f$ their chirp mass $\mathcal{M}$ is well below that of a typical detached system. But it was overlooked before that at higher $f$, where the number of AM CVn systems is much smaller, their $\mathcal{M}$ is similar to that of the detached systems from which they descend. It was shown that, out of the total population of $\sim 140,000 \mathrm{AM}$ CVn-stars with $P \leq 1500 \mathrm{~s}$, for $T_{\mathrm{obs}}=1 \mathrm{yr}$, LISA may be expected to resolve $\sim 11,000$ AM CVn systems at $S / N \geq 1$ (or $\sim 3,000$ at $S / N \geq 5$ ), i.e. the numbers of "resolvable" detached DDs and interacting DDs are similar. Given all uncertainties in the input data, these numbers are in reasonable agreement with the estimates of the numbers of potentially resolved detached DDs obtained by other authors, e.g., 3,000 to 6,000 [374, 62]. These numbers may be compared with expectation that $\sim 10$ Galactic NS + WD binaries will be detected [61].

The population of potentially resolved AM CVn-type stars is plotted in the right panel of Figure 15. Peculiarly enough, as a comparison of Figures 14 and 15 shows, the AM CVn-type systems appear, in fact, dominant among so-called "verification binaries" for LISA: binaries that are well known from electromagnetic observations and whose radiation is estimated to be sufficiently strong to be detected; see the list of 30 promising candidates in [390] and references therein, and the permanently updated (more rigorous) list of these binaries supported by G. Nelemans, G. Ramsay,

Living Reviews in Relativity

http: //www. livingreviews.org/lrr-2006-6 


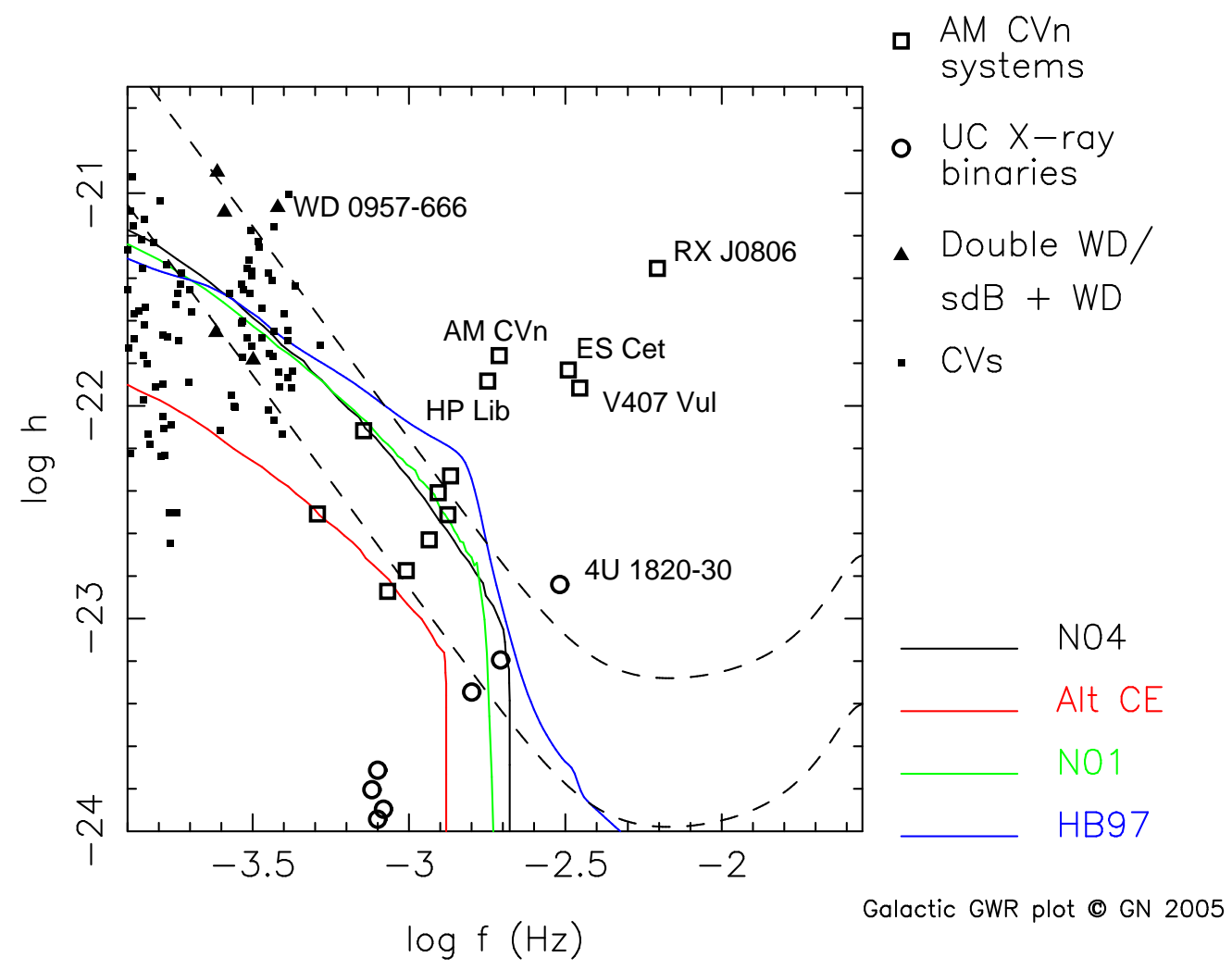

Figure 14: Gravitational waves background formed by the Galactic population of white dwarfs and signal amplitudes produced by some of the most compact binaries with white dwarf components ([272]). The green line presents results from [285], the black one from [286], while the red one presents a model with all assumptions similar to [286], but with the $\gamma$-formalism for the treatment of common envelopes [284, 283] (see also Section 3.5). The blue line shows the background derived in [27]. (Figure from [274].) 


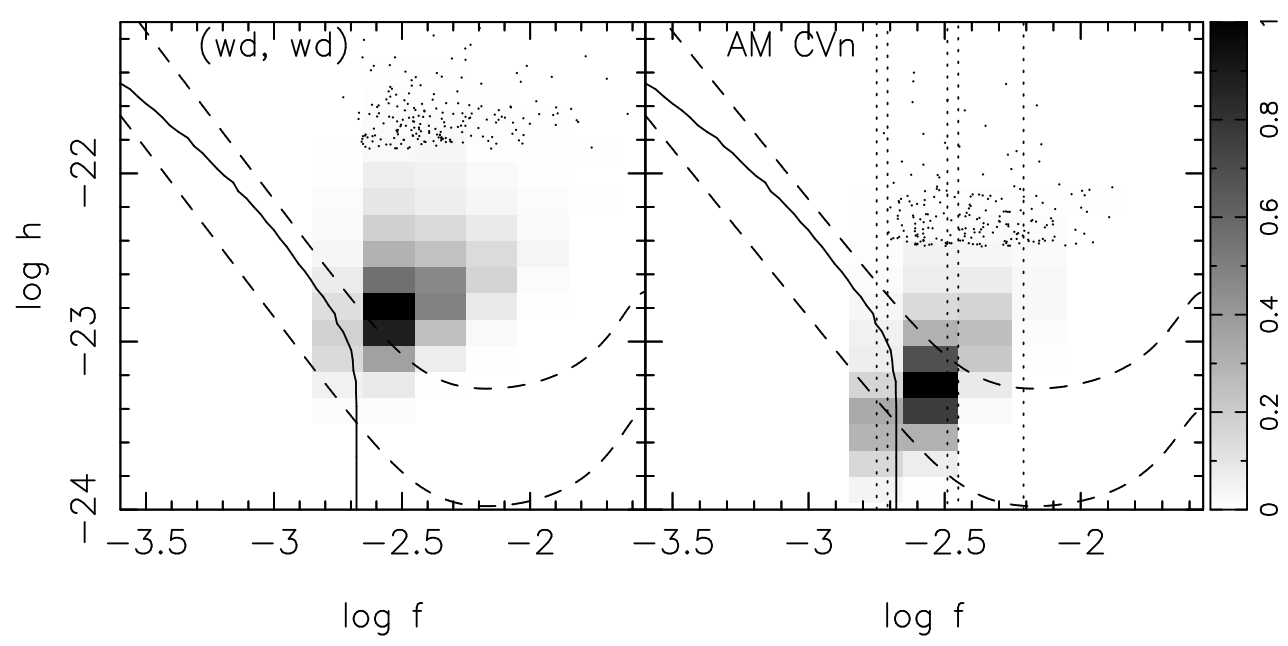

Figure 15: Strain amplitude $h$ as a function of the frequency for the model populations of resolved DDs (left panel, $\simeq 10,700$ objects) and AM CVn systems (right panel, $\simeq 11,000$ objects.). The gray shades give the density distribution of the resolved systems normalized to the maximum density in each panel (1,548 and 1,823 per "cell" for the double white dwarfs and AM CVn-stars panels, respectively). The 200 strongest sources in each sample are shown as dots to enhance their visibility. In the AM CVn panel the periods of several observed short period systems are indicated by the vertical dotted lines. The solid line shows the average background noise due to detached white dwarfs. The LISA sensitivities for an integration time of one year and a signal-to-noise ratio of 1 and 5 are indicated by the dashed lines [210]. (Figure from [286].)

and T. Marsh at [275].

Systems RXJ0806.3+1527, V407 Vul, ES Cet, and AM CVn are currently considered as the best candidates. We must note that the most severe "astronomical" problems concerning "verification binaries" are their distances, which for most systems are only estimates, and poorly constrained component masses. 


\section{AM CVn-Type Stars as Sources of Optical and X-ray Emission}

The circumstances mentioned in the previous paragraph stress the importance of studying AM CVnstars in all possible wavebands. LISA will measure a combination of all the parameters that determine the GWR signal (frequency, chirp mass, distance, position in the sky, and inclination angle; see, e.g., [139]), so if some of these parameters (period, position) can be obtained from optical or X-ray observations, the other parameters can be determined with higher accuracy. This is particularly interesting for the distances, inclinations, and masses of the systems, which are very difficult to measure with other methods.

In the optical, the total sample of AM CVn-type stars is expected to be dominated by longperiod members of the class due to emission of their disks. But the shortest periods AM CVn-type stars that are expected to be observed with LISA may be observed both in optical and X-rays thanks to high mass-transfer rates (see Figure 9). A model for electromagnetic-emission properties of the ensemble of the shortest orbital period $P \leq 1500 \mathrm{~s}$ was constructed by Nelemans et al. [286].

In [286], only systems with He-WD or "semidegenerate" He-star donors were considered (see Figure 5). Systems with donors descending from strongly evolved MS-stars were excluded from consideration, since their fraction in the orbital period range interesting for LISA is negligible. The "optimistic" model of [285] was considered, which assumes efficient spin-orbital coupling in the initial phase of mass-transfer and avoids edge-lit detonations of helium accreted at low $\dot{M}$. Average temperature and blackbody emission models in $V$-band and in the ROSAT $0.1-2.4 \mathrm{keV}$ $\mathrm{X}$-ray band were considered, taking into account interstellar absorption. The ROSAT band was chosen because of the discovery of AM CVn itself [424] and two candidate AM CVn systems as ROSAT sources (RXJ0806.3+1527 [168] and V407 Vul [261]) and because of the possibility for a comparison to the ROSAT all-sky survey.

One may identify four main emission sites: the accretion disc and boundary layer between the disc and the accreting white dwarf, the impact spot in the case of direct impact accretion, the accreting star, and the donor star.

Optical emission. The luminosity of the disk may be estimated as

$$
L_{\mathrm{disc}}=\frac{1}{2} G M \dot{m}\left(\frac{1}{R}-\frac{1}{R_{\mathrm{L} 1}}\right) \operatorname{erg~s}^{-1},
$$

with $M$ and $R$ being the mass and radius of the accretor, $R_{\mathrm{L} 1}$ being the distance of the first Lagrangian point to the centre of the accretor, and $\dot{m}$ being the mass transfer rate, respectively. Optical emission of the disk was modeled as that of a single temperature disc that extends to $70 \%$ of the Roche lobe of the accretor and radiates as a blackbody [438].

The emission from the donor was treated as the emission of a cooling white dwarf, using approximations to the cooling models of Hansen [137].

The emission from the accretor was treated as the unperturbed cooling luminosity of the white $\operatorname{dwarf}^{16}$.

A magnitude-limited sample was considered, with $V_{\lim }=20 \mathrm{mag}$, typical for observed shortperiod AM CVn-type stars. Interstellar absorption was estimated using Sandage's model [366] and Equation (67).

X-ray emission. Most AM CVn systems experience a short $\left(10^{6}-10^{7}\right.$ yr) "direct impact" stage in the beginning of mass-transfer [142, 285, 249]. Hence, in modeling the X-ray emission of AM CVn systems one has to distinguish two cases: direct impact and disk accretion.

\footnotetext{
${ }^{16}$ This is true for short-period systems, but becomes an oversimplification at longer periods and for $P_{\text {orb }} \gtrsim 40$ min heating of the WD by accretion has to be taken into account [34].
} 
In the case of a direct impact a small area of the accretor's surface is heated. One may assume that the total accretion luminosity is radiated as a blackbody with a temperature given by

$$
\left(\frac{T_{\mathrm{BB}}}{T_{\odot}}\right)^{4}=\frac{1}{s} R^{-2} L_{\mathrm{acc}}
$$

where $L_{\text {acc }}$ and $R$ are in solar units and $L_{\text {acc }}$ is defined by Equation (68). The fraction $s$ of the surface that is radiating depends on the details of the accretion. It was set to 0.001, consistent with expectations for a ballistic stream [238] and the observed X-ray emission of V407 Vul, a known direct-impact system [251].

In the presence of a disk, X-ray emission was assumed to be coming from a boundary layer with temperature [333]

$$
T_{\mathrm{BL}}=5 \times 10^{5}\left(\frac{\dot{m}}{10^{18} \mathrm{~g} \mathrm{~s}^{-1}}\right)^{\frac{2}{9}}\left(\frac{M}{M_{\odot}}\right)^{\frac{1}{3}}\left(\frac{R}{5 \times 10^{8} \mathrm{~cm}}\right)^{-\frac{7}{9}} \mathrm{~K} .
$$

The systems with an X-ray flux in the ROSAT band higher than $10^{-13} \mathrm{erg} \mathrm{s}^{-1} \mathrm{~cm}^{-2}$ were selected. Then, the intrinsic flux in this band, the distance and an estimate of the Galactic hydrogen absorption [260] provide an estimate of detectable flux.

Figure 16 presents the resulting model. In the top panel there are 220 systems only detectable in X-rays and 330 systems also detectable in the $V$-band. One may distinguish two subpopulations in the top panel: In the shortest period range there are systems with white-dwarf donors with such high $\dot{M}$ that even sources close to the Galactic centre are detectable. Spatially, these objects are concentrated in a small area on the sky. At longer periods the X-rays get weaker (and softer) so only the systems close to the Earth can be detected. They are more evenly distributed over the sky. Several of these systems are also detectable in the optical (filled symbols). There are 30 systems that are close enough to the Earth that the donor stars can be seen as well as the discs (filled squares). Above $P=600 \mathrm{~s}$ the systems with helium-star donors show up and have a high enough mass transfer rate to be X-ray sources, the closer ones of which are also visible in the optical, as these systems always have a disc. The bottom panel shows the 1,230 "conventional" AM CVn systems, detectable only by optical emission, which for most systems emanates only from their accretion disc. Of this population 170 objects closest to the Earth also have a visible donor. The majority of the optically detectable systems with orbital periods between 1,000 and 1,500 s are expected to show outbursts due to the viscous-thermal disc instability [406] which could enhance the chance of their discovery.

Living Reviews in Relativity

http: //www . livingreviews . org/lrr-2006-6 


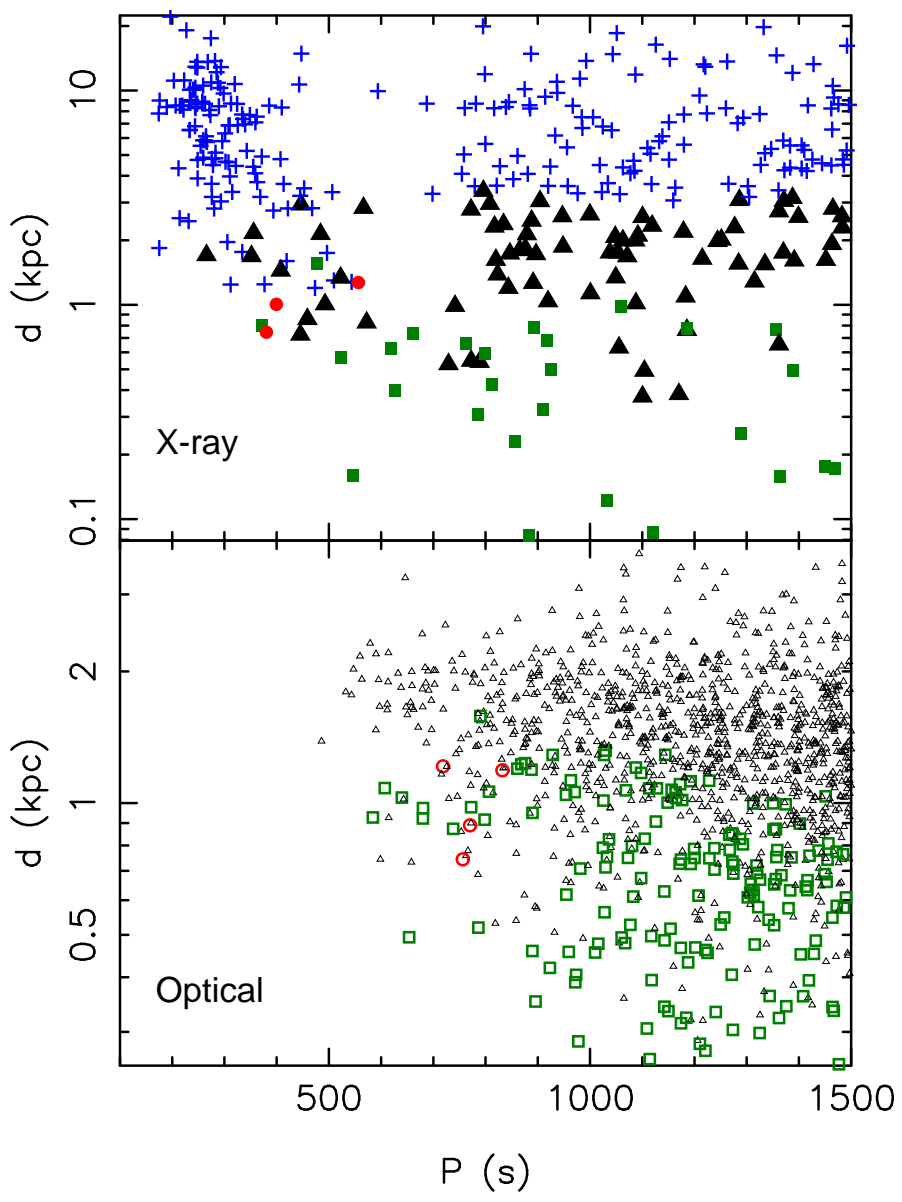

Figure 16: Distribution of short period AM CVn-type systems detectable in soft X-rays and as optical sources as a function of orbital period and distance. Top panel: systems detectable in $X$ rays only (blue pluses), direct impact systems observable in $X$-ray and $V$-band (red filled circles), systems detectable in X-ray with an optically visible donor (green squares), and systems detectable in X-rays and with an optically visible disc (large filled triangles). Bottom panel: direct impact systems (red open circles), systems with a visible donor (green squares), and systems with a visible accretion disc (small open triangles). The overlap of these systems with systems observable in gravitational waves is shown in Figure 17. (Updated figure from [286], see also [273].) 


\section{AM CVn-Type Stars Detectable in GWR and Electro- magnetic Spectrum}

Figure 17 [286] shows the distributions vs. orbital periods for the total number of AM CVn systems with $P \leq 1500 \mathrm{~s}$ and for AM CVn LISA sources that have optical and/or X-ray counterparts. The interrelations between numbers of sources emitting in different wavebands are shown in the legend to the right of the figure. Out of 11,000 systems detectable in GWR, 2,060 are expected to be in the direct-impact (DI) stage and only 325 in the mass-transfer via disk stage. Thus, the majority of the DI systems are expected to be detectable in GWR; some $5 \%$ of DI systems are expected to emit X-rays. There are 1,336 systems detectable in the optical waveband and 326 in X-rays; 106 members of the latter samples may be detected in both spectral ranges.

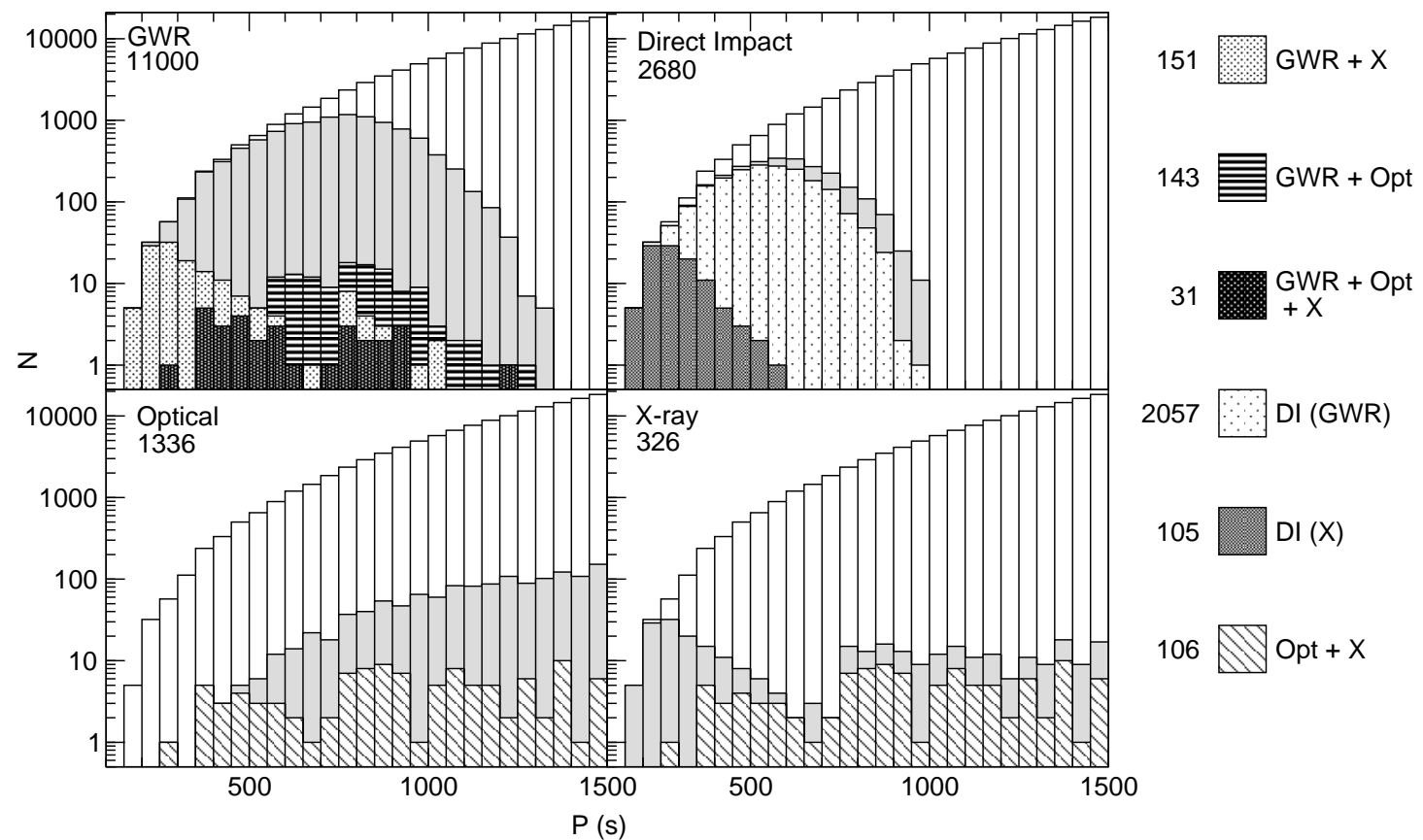

Figure 17: Short-period AM CVn systems, subdivided in different types. Each panel shows the total population as the white histogram. The top left panel shows 11,000 systems that can be resolved by LISA in gray, and they are subdivided into the ones that have optical counterparts (GWR + Opt), $X$-ray counterparts $(G W R+X)$, and both $(G W R+O p t+X)$. The top right panel shows the systems that are in the direct impact phase of accretion in gray, and they are subdivided in $G W R$ and $X$-ray sources. The bottom two panels show (again in gray) the populations that are detectable in the optical band (left panel) and the X-ray band (right panel). The distribution of sources detectable both in optical and X-ray bands is shown as hatched bins in both lower panels (Opt $+X)$. (Figure from [286].)

An additional piece of information may be obtained from eclipsing AM CVn-stars: They would provide radii of the components and orbital inclinations of the systems. A systematical study of the possibility of eclipses was never carried out, but an estimate for a "typical" system with initial masses of components $(0.25+0.60) M_{\odot}$ shows that the probability for eclipsing of the accretor is about $30 \%$ at $P=1000 \mathrm{~s}$, and even higher for eclipsing (a part of) the accretion disc. The first detection of an eclipsing AM CVn-type star - SDSS J0926+3624 ( $P_{\text {orb }}=28.3$ min $)$ - was recently

Living Reviews in Relativity

http: //www . livingreviews . org/lrr-2006-6 
reported by Anderson et al. [8].

For WD + WD pairs detectable by LISA the prospects of optical identification are negligible, since for them cooling luminosity is the only source of emission. Most of the potentially detectable dwarfs are located close to the Galactic center and will be very faint. Estimates based on the model [286] predict for the bulk of them $V \approx 35 \mathrm{mag}$, with only 75 objects detectable with $V<25$ mag. Even inclusion of brightening of the dwarfs close to contact under the assumption of efficient tidal heating [164] increases this number to $\approx 130$ only (G. Nelemans, private communication).

In the discussion above, we considered the X-ray flux of AM CVn-type systems in the ROSAT waveband: $0.1-2.4 \mathrm{keV}$. It may be compared with the expected flux in the Chandra and XMM bands: $0.1-15 \mathrm{keV}$. Since most of the spectra of model AM CVn-stars are rather soft, the flux $\mathcal{F}$ in the latter band is generally not much larger than in the ROSAT band: $80 \%$ of systems have $\mathcal{F}_{\text {XMM }} / \mathcal{F}_{\text {ROSAT }}<1.5 ; 96 \%$ have $\mathcal{F}_{\text {XMM }} / \mathcal{F}_{\text {ROSAT }}<3$. However, Chandra and XMM have much higher sensitivity. For instance, the Chandra observations of the Galactic centre have a completeness limit of $3 \times 10^{-15} \mathrm{erg} \mathrm{cm}^{-2} \mathrm{~s}^{-1}$, almost two orders of magnitude deeper than our assumed ROSAT limit [262]. The expected number of X-ray sources in the $0.1-15 \mathrm{keV}$ band detectable down to $10^{-14} \mathrm{erg} \mathrm{cm}^{-2} \mathrm{~s}^{-1}$ is 644 and it is 1085 down to $10^{-15} \mathrm{erg} \mathrm{cm}^{-2} \mathrm{~s}^{-1}$. In the Chandra mosaic image of the Galactic centre [441], roughly down to $10^{-14} \mathrm{erg} \mathrm{cm}^{-2} \mathrm{~s}^{-1}$ there are $\sim 1,000$ point sources, presumably associated with accreting white dwarfs, neutron stars, and black holes. Model [286] predicts 16 X-ray systems in this region.

\subsection{Effects of finite entropy}

Results concerning the AM CVn-stars population presented above were obtained assuming a massradius relation for zero-temperature WDs. Evidently, this is a quite crude approximation, having in mind that in some cases the time span between the emergence of the second white dwarf from the common envelope and contact may be as short as several Myr [422]. As the first step to more realistic models, Deloye and coauthors [76] considered the effects of finite entropy of the donors by using their finite-entropy models for white dwarfs [75]. We illustrate some of these effects following [76].

The effects of finite entropy become noticeably important for $M \lesssim 0.1 M_{\odot}$. Isentropic WD with $T>0$ (i) have larger radii than $T=0$ objects and (ii) the $M-R$ relations for them are steeper than for $T=0$ (i.e. in the range of interest they are still negative but have a lower absolute value). By virtue of Equations $(63,64)$ this means that for a given orbital period they have higher $\dot{M}$. This effect is illustrated in the left panel of Figure 18. (The period-mass relation is not single-valued, since the $M-R$ relation has two branches: a branch where the object is thermally supported and a branch where degenerate electrons provide the dominant pressure support.) The right panel of Figure 18 compares a model of the population of AM CVn-stars computed under assumptions that the donor white dwarfs have $T=0$ and a model which takes into account cooling of the prospective donors between formation and RLOF. The change in the rate of evolution (shown in the left panel) shifts systems with "realistic" cooling to longer orbital periods as compared to the $T=0$ population.

Finite entropy of the donors also influences the gravitational waves signals from AM CVn-stars. Again, by virtue of the requirement of $R_{\text {donor }}=R_{\mathrm{L}}$, the systems with $T=0$ donors and hot donors will have a different $P_{\text {orb }}$ for the same combination of component masses, i.e. different radii at the contact and different relation between chirp mass $\mathcal{M}$ and $P_{\text {orb }}$. This alters the GW amplitude, Equation (17.

For instance, if a $0.2 M_{\odot}$ donor is fully degenerate, it overflows its Roche lobe at $P_{\text {orb }} \approx 3.5 \mathrm{~min}$ and then evolves to longer $P_{\text {orb }}$. If "realistic" cooling is considered, there are donors that make contact at $P_{\text {orb }}$ up to $\approx 25$ min. Hotter donors at fixed $P_{\text {orb }}$ are more massive, increasing $\mathcal{M}$ and 

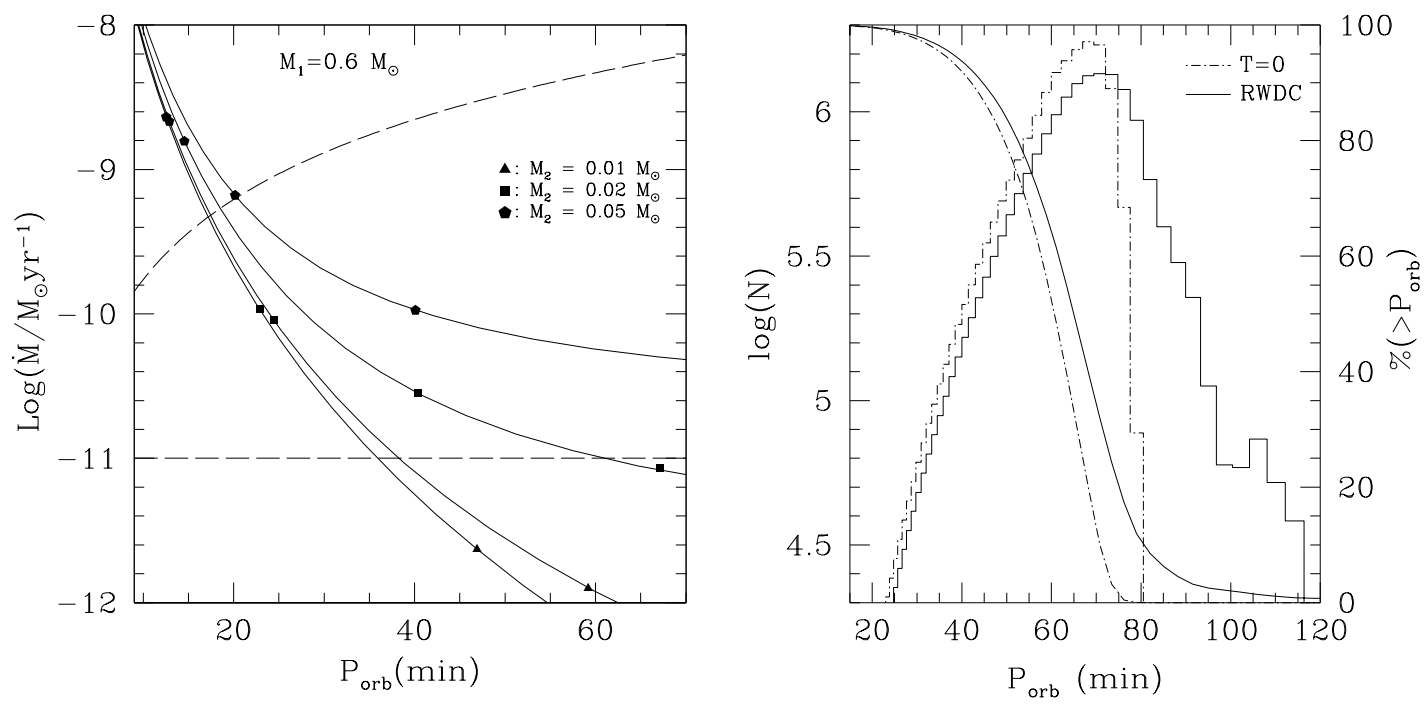

Figure 18: Effects of a finite entropy of donors on the properties of AM CVn-stars. The left panel shows the relation between $P_{\text {orb }}$ and $\dot{M}$ along tracks for a system with initial masses of components $0.2 M_{\odot}$ and $0.6 M_{\odot}$ (like in Figure 9). The solid lines show the evolution for donors with $T_{\mathrm{c}}=10^{4} \mathrm{~K}, 10^{6} \mathrm{~K}, 5 \times 10^{6} \mathrm{~K}$, and $10^{7} \mathrm{~K}$ (left to right). The symbols show the positions of models with $M_{2}=0.01 M_{\odot}$ (triangles), $0.02 M_{\odot}$ (squares), and $0.05 M_{\odot}$ (pentagons). The disk stability criteria (for $q=0.05$ ) are shown by the dashed lines (after [406]). The right panel compares the numbers of systems as a function of $P_{\text {orb }}$ for the model with a $T=0 \mathrm{WD}$ (dot-dashed line) and the model with "realistic" cooling (solid lines, $R W D C$ ). The smooth curves show the percentage of each population laying above a given $P_{\text {orb. }}$. (Figures from [76].) 
increasing $h$. Thus, the contribution of the individual systems to the integrated GW flux from the total ensemble increases, but their higher rate of evolution decreases the density of population of the sources detectable at low $f$, since they are lost in the background confusion noise of Galactic WDs. But altogether, the ensemble of sources detectable by LISA with $S / N>1$ diminishes by about $10 \%$ only. Note that finite entropy of donors does not significantly affect the properties of the $\sim 10,000$ systems that are expected to be observed both in electromagnetic spectrum and gravitational waves.

There are some more subtle effects related to finite entropy for which we refer the reader to the original paper.

\subsection{Going further}

The major issue concerning compact binaries - DDs, IDDs, UCXBs - is their number. Theoretical predictions strongly depend on the assumed parameters and range within an order of magnitude (see references in Section 10). The treatment of common envelopes and the distribution of stars over $q$ are, perhaps, the crux. On the other hand, observational estimates suffer from numerous selection effects. For instance, the estimates of the local space density of white dwarfs may be uncertain by a factor of $\sim 5$ : cf. $4.2 \times 10^{-3} \mathrm{pc}^{-3}$ [193], $(5 \pm 0.7) \times 10^{-3} \mathrm{pc}^{-3}$ [149], or $(20 \pm 7) \times 10^{-3} \mathrm{pc}^{-3}$ [103]. The problem with AM CVn-stars is their deficiency by a factor of several compared even to the most pessimistic predictions, but in this case the situation improves: about half of the known and candidate systems were found within the past decade, while for another half it took about 30 years. In contrast to DDs, systematic searches for AM CVn-stars started only recently; in fact, the majority of known objects were found serendipitously. Rather successful was the search for AM CVn-candidates in the SDSS catalogue and follow-up observations which brought two confirmed and three candidate stars [353, 8]. Further progress may be expected from dedicated surveys with wide-field cameras. "RApid Temporal Survey" (RATS) by Ramsey and Hakala [338] is aimed at discovering objects with periodicities down to $2 \mathrm{~min}$ and is sensitive to $V \sim 22.5$. Preliminary results [336] yielded no new AM CVn-stars, though several objects were expected in the 6 square degrees of the sky covered so far. This may be related to the fact that initially the interstellar extinction for these objects, which have to be located predominantly in the Galactic plane, was underestimated.

Another promising project is "OmegaWhite" (with PI P. Groot, University of Nijmegen in the Netherlands); see [123].

This project is also aimed at compact binaries, it will cover 400 square degrees of the sky, and will provide broad-band and narrow band photometric information down to 21st magnitude or equivalent line fluxes.

Another key issue in the studies of detached and interacting binary white dwarfs is the determination of their distances. In this aspect, the Gaia space probe which is aimed at the creation of a three-dimensional chart of our Galaxy by providing positional and radial velocity measurements for about one billion stars (see [91]) may appear the most promising source of information.

A failure to discover a significant number of detached and interacting double degenerates or to confirm current ideas on their structure and evolution will mean that serious drawbacks exist either in the implementation of known stellar evolution physics and observational statistical data in the population synthesis codes or in our understanding of the processes occurring in compact binaries or in the treatment of selection effects. Special attention in theoretical studies has to be paid to the onset of mass-transfer.

Above, we presented some of the current ideas on formation and compact binaries that may be interesting for general relativity and cosmology and on signals that may be expected from them in the LISA waveband. There is another side of the problem - the analysis of the signal, would it be detected. This topic is out of the scope of this brief review. We refer the reader only to 
several papers discussing methods for detecting and subtracting individual binary signals from a data stream with many overlapping signals [63], inferring properties of the distribution of whitedwarf binaries [88], the accuracy of parameter estimation of low-mass binaries [396, 355], and the discussion of the quality of information that may be provided by LISA [391]. 


\section{Conclusions}

The current understanding of the evolution of binary stars is firmly based on observations of many types of binary systems, from wide non-interacting pairs to very close compact binaries consisting of remnants of stellar evolution. The largest uncertainties in the specific parameters of the double compact binary formed at the end of the evolution of a massive binary system are related to the physical properties of the pre-supernovae: masses, magnetic fields, equation of state (for NSs), spins, possible kick velocities, etc. This situation is due to our limited understanding of both the late stages of stellar evolution and especially of the supernovae explosion mechanisms. The understanding of the origin and evolution of compact white dwarf binaries also suffers from incompleteness of our knowledge of white dwarf formation and, in particular, on the common envelope treatment. The progress in these fields, both observational and theoretical, will have a major effect on the apprehension of the formation and evolution of compact binary systems. On the other hand, the phenomenological approach used to describe these uncertainties proves to be successful in explaining many observed properties of various binary stars, so the constraints derived from the studies of binary stars should be taken into account in modeling stellar evolution and supernovae explosions.

Of course, specifying and checking the initial distributions of orbital parameters of binary stars and parameters of binary evolution (such as evolution in the common envelopes) stay in the shortlist of the important actions to be done. Here an essential role belongs to detailed numerical simulations.

Further observations of compact binaries. Clearly, discoveries of new types of compact binary systems have provided the largest impetus for studies of binary star evolution. The well known examples include the discovery of X-ray binaries, relativistic binary pulsars, and millisecond recycled pulsars. In the nearest future we expect the discovery of NS $+\mathrm{BH}$ binaries which are predicted by the massive binary evolution scenario in the form of binary radio pulsars with BH companions $[228,220,309]$. It is very likely that we already observe the coalescence of double NS/BH systems as short gamma-ray bursts in other galaxies [116]. We also anticipate that coalescences of $\mathrm{NS}+\mathrm{BH}$ or $\mathrm{BH}+\mathrm{BH}$ binaries can be found first in GW data analysis [122]. The efforts of the LIGO collaboration to put constraints on the compact binary coalescences from the analysis of the existing GW observations are very important [3, 1, 2].

The formation and evolution of compact binaries is a very interdisciplinary field of modern astrophysics, ranging from studies of the equation of state for superdense matter inside neutron stars and testing effects of strong gravity in relativistic compact binaries to hydrodynamical effects in stellar winds and formation of common envelopes. So, further progress in this field, which will be made by means of traditional astronomical observations and new tools, like gravitational wave and neutrino detectors, will undoubtedly have an impact on astronomy and astrophysics as a whole. 


\section{Acknowledgments}

The authors acknowledge the referee for careful reading of the manuscript and useful comments. The authors would like to thank L.P. Grishchuk, V.M. Lipunov, M.E. Prokhorov, A.G. Kuranov, A. Tutukov, M. Livio, G. Dubus, J.-P. Lasota, E.P.J. van den Heuvel, and R. Napiwotzki for numerous useful discussions and joint research in the evolution of binary stars. Writing of this review would have been impossible without the long-term research cooperation with G. Nelemans. We acknowledge him also for useful discussions, help in collecting information, and updating figures. Useful discussions with participants of the First Nijmegen Workshop on AM CVn-stars (July 2005) are acknowledged. We thank P. Groot for providing information on the "OmegaWhite"project. An intensive use of the Smithsonian/NASA ADS Astronomy Abstract Service and arXiv is acknowledged. The work is partially supported by the RFBR grants 06-02-16025, 04-02-16720 and by Russian Academy of Sciences Basic Research Program "Origin and Evolution of Stars and Galaxies". 


\section{References}

[1] Abbott, B., Abbott, R., Adhikari, R., Ageev, A., Agresti, J., Ajith, P., Allen, B., Allen, J., Amin, R., Anderson, S.B., and coauthors, 422, "Search for gravitational waves from binary black hole inspirals in LIGO data", Phys. Rev. D, 73, 1-17, 062001, (2006). Related online version (cited on 4 November 2006):

http://adsabs.harvard.edu/abs/2006PhRvD. .73f2001A. 13

[2] Abbott, B., Abbott, R., Adhikari, R., Ageev, A., Agresti, J., Ajith, P., Allen, B., Allen, J., Amin, R., Anderson, S.B., and coauthors, 519, "Joint LIGO and TAMA300 search for gravitational waves from inspiralling neutron star binaries", Phys. Rev. D, 73, 1-10, 102002, (2006). Related online version (cited on 4 November 2006): http://adsabs.harvard.edu/abs/2006PhRvD. .73j2002A. 13

[3] Abbott, B. et al. (LIGO Scientific Collaboration), "Analysis of LIGO data for gravitational waves from binary neutron stars", Phys. Rev. D, 69, 1-16, 122001, (2004). 2.2, 13

[4] Abt, H.A., "Normal and abnormal binary frequencies", Annu. Rev. Astron. Astrophys., 21, $343-372,(1983) .5 .1$

[5] Acernese, F. et al. (VIRGO Collaboration), "Status of Virgo", Class. Quantum Grav., 22, S869-S880, (2005). Related online version (cited on 21 June 2006):

http://arXiv.org/abs/gr-qc/0406123. Proceedings of the 9th Gravitational Wave Data Analysis Workshop, Annecy, France, 15-18 December 2004. 1

[6] Alexander, M.E., Chau, W.Y., and Henriksen, R.N., "Orbital evolution of a singly condensed, close binary, by mass loss from the primary and by accretion drag on the condensed member", Astrophys. J., 204, 879-888, (1976). Related online version (cited on 21 June 2006): http://adsabs.harvard.edu/abs/1976ApJ . . 204 . .879A. 3.5

[7] Anderson, S.B., Gorham, P.W., Kulkarni, S.R., Prince, T.A., and Wolszczan, A., "Discovery of two radio pulsars in the globular cluster M15", Nature, 346, 42-44, (1990). 2.1

[8] Anderson, S.F., Haggard, D., Homer, L., Joshi, N.R., Margon, B., Silvestri, N.M., Szkody, P., Wolfe, M.A., Agol, E., Becker, A.C., Henden, A., Hall, P.B., Knapp, G.R., Richmond, M.W., Schneider, D.P., Stinson, G., Barentine, J.C., Brewington, H.J., Brinkmann, J., Harvanek, M., Kleinman, S.J., Krzesinski, J., Long, D., Neilsen, E.H., Nitta, A., and Snedden, S.A., "Ultracompact AM Canum Venaticorum Binaries from the Sloan Digital Sky Survey: Three Candidates Plus the First Confirmed Eclipsing System", Astron. J., 130, 2230-2236, (2005). Related online version (cited on 21 June 2006): http://adsabs.harvard.edu/abs/2005AJ....130.2230A. 12, 12.2

[9] Ashok, N.M., "Infrared study of the first identified helium nova V445 Puppis", Bull. Astr. Soc. India, 33, 75, (2005). Related online version (cited on 21 June 2006): http://adsabs.harvard.edu/abs/2005BASI ...33...75A. 7

[10] Ashok, N.M., and Banerjee, D.P.K., "The enigmatic outburst of V445 Puppis - A possible helium nova?", Astron. Astrophys., 409, 1007-1015, (2003). Related online version (cited on 21 June 2006):

http://adsabs.harvard.edu/abs/2003A\&A...409.1007A. 7

[11] Babak, S.V., and Grishchuk, L.P., "Energy-momentum tensor for the gravitational field", Phys. Rev. D, 61, 024038, (2000). 3.1 .3 
[12] Bagot, P., "Boost of the orbital motion in high mass X-ray binaries", Astron. Astrophys., 314, 576-584, (1996). 3.5

[13] Bagot, P., "On the progenitors of double neutron star systems", Astron. Astrophys., 322, $533-544,(1997)$. 4.1

[14] Bagot, P., Portegies Zwart, S.F., and Yungelson, L.R., "Gamma-ray bursts and density evolution of neutron star binary mergers", Astron. Astrophys., 332, L57-L60, (1998). Related online version (cited on 4 November 2006):

http://adsabs.harvard.edu/abs/1998A\&A. . .332L . 57B. 2.3

[15] Baraffe, I., Heger, A., and Woosley, S.E., "On the Stability of Very Massive Primordial Stars", Astrophys. J., 550, 890-896, (2001). Related online version (cited on 21 June 2006): http://adsabs.harvard.edu/abs/2001ApJ ...550..890B. 1

[16] Barish, B.C., and Weiss, R., "LIGO and the detection of gravitational waves", Phys. Today, 52, 44-50, (1999). Related online version (cited on 21 June 2006):

http://adsabs.harvard.edu/abs/1999PhT....52j . .44B. 1

[17] Barkov, M.V., Bisnovatyi-Kogan, G.S., and Lamzin, S.A., "The Thermal Evolution of Thorne-Zytkow Objects", Astron. Rep., 45, 230-235, (2001). Related online version (cited on 21 June 2006):

http://adsabs.harvard.edu/abs/2001ARep...45..230B. 6

[18] Barthelmy, S.D., Chincarini, G., Burrows, D.N., Gehrels, N., Covino, S., Moretti, A., Romano, P., O’Brien, P.T., Sarazin, C.L., Kouveliotou, C., Goad, M., Vaughan, S., Tagliaferri, G., Zhang, B., Antonelli, L.A., Campana, S., Cummings, J.R., D’Avanzo, P., Davies, M.B., Giommi, P., Grupe, D., Kaneko, Y., Kennea, J.A., King, A., Kobayashi, S., Melandri, A., Mészáros, P., Nousek, J.A., Patel, S., Sakamoto, T., and Wijers, R.A.M.J., "An origin for short $\gamma$-ray bursts unassociated with current star formation", Nature, 438, 994-996, (2005). Related online version (cited on 21 June 2006):

http://adsabs.harvard.edu/abs/2005Natur.438 . 994B. 2.3

[19] Baryshev, Y.V., and Paturel, G., "Statistics of the detection rates for tensor and scalar gravitational waves from the Local Galaxy universe", Astron. Astrophys., 371, 378-392, (2001). Related online version (cited on 4 November 2006): http://adsabs.harvard.edu/abs/2001A\&A...371..378B. 6.2

[20] Bassa, C.G., Jonker, P.G., in’t Zand, J.J.M., and Verbunt, F., "Two new candidate ultracompact X-ray binaries", Astron. Astrophys., 446, L17-L20, (2006). Related online version (cited on 21 June 2006):

http://adsabs.harvard.edu/abs/2006A\&A...446L ..17B. 8

[21] Belczynski, K., Bulik, T., and Kalogera, V., "Merger Sites of Double Neutron Stars and Their Host Galaxies", Astrophys. J. Lett., 571, L147-L150, (2002). 2.3

[22] Belczynski, K., Kalogera, V., and Bulik, T., "A Comprehensive Study of Binary Compact Objects as Gravitational Wave Sources: Evolutionary Channels, Rates, and Physical Properties", Astrophys. J., 572, 407-431, (2002). Related online version (cited on 21 June 2006): http://adsabs.harvard . edu/abs/2002ApJ . .572 . .407B. 2.2, 3.4, 6, 4.1

[23] Belczynski, K., Kalogera, V., Rasio, F.A., Taam, R.E., Zezas, A., Bulik, T., Maccarone, T.J., and Ivanova, N., "Compact Object Modeling with the StarTrack Population Synthesis Code", Astrophys. J. Suppl. Ser., submitted, (2005). Related online version (cited on 21 June 
2006):

http://arXiv.org/abs/astro-ph/0511811. 5.2

[24] Belczynski, K., Perna, R., Bulik, T., Kalogera, V., Ivanova, N., and Lamb, D.Q., "A Study of Compact Object Mergers as Short Gamma-Ray Burst Progenitors", Astrophys. J., 648, 1110-1116, (2006). Related online version (cited on 4 November 2006): http://adsabs.harvard.edu/abs/2006ApJ ...648.1110B. 2.3

[25] Belczynski, K., and Taam, R.E., "Galactic Populations of Ultracompact Binaries", Astrophys. J., 603, 690-696, (2004). Related online version (cited on 21 June 2006):

http://adsabs.harvard.edu/abs/2004ApJ ...603..690B. 7

[26] Benacquista, M., "Relativistic Binaries in Globular Clusters", Living Rev. Relativity, 9, lrr2006-2, (2006). URL (cited on 21 June 2006):

http://www.livingreviews.org/lrr-2006-2. 2.2, 4.1, 7

[27] Bender, P.L., and Hils, D., "Confusion noise level due to galactic and extragalactic binaries", Class. Quantum Grav., 14, 1439-1444, (1997). 14

[28] Benz, W., Bowers, R.L., Cameron, A.G., and Press, W.H., "Dynamic mass exchange in doubly degenerate binaries. I. 0.9 and 1.2 Mo stars", Astrophys. J., 348, 647-667, (1990). 7

[29] Berger, E., "The Afterglows and Host Galaxies of Short GRBs: An Overview", in Holt, S.S., Gehrels, N., and Nousek, J.A., eds., Gamma-Ray Bursts in the Swift Era, Sixteenth Maryland Astrophysics Conference, Washington, DC, 29 November - 2 December 2005, vol. 836 of AIP Conference Proceedings, (American Institute of Physics, Melville, U.S.A., 2006). Related online version (cited on 21 June 2006):

http://adsabs.harvard.edu/abs/2006astro.ph. .2004B. 2.3, 4

[30] Berger, E., Fox, D.B., Price, P.A., Nakar, E., Gal-Yam, A., Holz, D.E., Schmidt, B.P., Cucchiara, A., Cenko, S.B., Kulkarni, S.R., Soderberg, A.M., Frail, D.A., Penprase, B.E., Rau, A., Ofek, E., Bell Burnell, S.J., Cameron, P.B., Cowie, L.L., Dopita, M.A., Hook, I., Peterson, B.A., Podsiadlowski, P., Roth, K.C., Rutledge, R.E., Sheppard, S.S., and Songaila, A., "A New Population of High Redshift Short-Duration Gamma-Ray Bursts", Astrophys. $J$. , submitted, (2006). Related online version (cited on 4 November 2006):

http://arXiv.org/abs/astro-ph/0611128. 2.3

[31] Berger, E., Price, P.A., Cenko, S.B., Gal-Yam, A., Soderberg, A.M., Kasliwal, M., Leonard, D.C., Cameron, P.B., Frail, D.A., Kulkarni, S.R., Murphy, D.C., Krzeminski, W., Piran, T., Lee, B.L., Roth, K.C., Moon, D.-S., Fox, D.B., Harrison, F.A., Persson, S.E., Schmidt, B.P., Penprase, B.E., Rich, J., Peterson, B.A., and Cowie, L.L., "The afterglow and elliptical host galaxy of the short $\gamma$-ray burst GRB 050724", Nature, 438, 988-990, (2005). Related online version (cited on 21 June 2006):

http://adsabs.harvard.edu/abs/2005Natur.438..988B. 2.3

[32] Bethe, H.A., and Brown, G.E., "Evolution of Binary Compact Objects That Merge", Astrophys. J., 506, 780-789, (1998). 4.2, 5.2

[33] Bhattacharya, D., and van den Heuvel, E.P.J., "Formation and evolution of binary and millisecond radio pulsars", Phys. Rep., 203, 1-124, (1991). 3

[34] Bildsten, L., Townsley, D.M., Deloye, C.J., and Nelemans, G., "The Thermal State of the Accreting White Dwarf in AM Canum Venaticorum Binaries", Astrophys. J., 640, 466-473, (2006). Related online version (cited on 21 June 2006):

http://adsabs.harvard.edu/abs/2006ApJ ...640 . 466B. 16 
[35] Bisnovatyi-Kogan, G.S., Stellar Physics, Vol. 2: Stellar Evolution and Stability, (Springer, Berlin, Germany; New York, U.S.A., 2002). 1

[36] Bisnovatyi-Kogan, G.S., "Binary and recycled pulsars: 30 years after observational discovery", Phys. Usp., 176, 53-68, (2006). Related online version (cited on 21 June 2006):

http://adsabs.harvard.edu/abs/2005A\&AT ..24..151R. 5, 4.1

[37] Bisnovatyi-Kogan, G.S., and Komberg, B.V., "Pulsars and close binary systems", Sov. Astron., 18, 217-221, (1974). Related online version (cited on 21 June 2006):

http://adsabs.harvard.edu/abs/1974SvA....18..217B. 5

[38] Blaauw, A., "On the origin of the O- and B-type stars with high velocities (the "run-away" stars), and some related problems", Bull. Astron. Inst. Neth., 15, 265-290, (1961). 3.3

[39] Blinnikov, S.I., Novikov, I.D., Perevodchikova, T.V., and Polnarev, A.G., "Exploding Neutron Stars in Close Binaries", Sov. Astron. Lett., 10, 177, (1984). 2.3

[40] Bloom, J.S., Prochaska, J.X., Pooley, D., Blake, C.H., Foley, R.J., Jha, S., Ramirez-Ruiz, E., Granot, J., Filippenko, A.V., Sigurdsson, S., Barth, A.J., Chen, H.-W., Cooper, M.C., Falco, E.E., Gal, R.R., Gerke, B.F., Gladders, M.D., Greene, J.E., Hennanwi, J., Ho, L.C., Hurley, K., Koester, B.P., Li, W., Lubin, L., Newman, J., Perley, D.A., Squires, G.K., and WoodVasey, W.M., "Closing in on a Short-Hard Burst Progenitor: Constraints from Early-Time Optical Imaging and Spectroscopy of a Possible Host Galaxy of GRB 050509b", Astrophys. J., 638, 354-368, (2006). Related online version (cited on 21 June 2006):

http://adsabs.harvard.edu/abs/2006ApJ . .638. .354B. 2.3

[41] Bragaglia, A., Greggio, L., Renzini, A., and D'Odorico, S., "Double Degenerates among DA white dwarfs", Astrophys. J., 365, L13-L17, (1990). 8

[42] Brandt, N., and Podsiadlowski, P., "The effects of high-velocity supernova kicks on the orbital properties and sky distribution of neutron", Mon. Not. R. Astron. Soc., 274, 461-484, (1995). 3.3

[43] Brandt, N., and Podsiadlowski, P., "The effects of high-velocity supernova kicks on the orbital properties and sky distributions of neutron-star binaries", Mon. Not. R. Astron. Soc., 274, 461-484, (1995). 3.4.1

[44] Brown, G.E., Lee, C.-H., Wijers, R.A.M.J., and Bethe, H.A., "Evolution of black holes in the Galaxy", Phys. Rep., 333, 471-504, (2000). Related online version (cited on 4 November 2006):

http://adsabs.harvard.edu/abs/2000PhR...333..471B. 6

[45] Brumberg, V.A., Zeldovich, I.B., Novikov, I.D., and Shakura, N.I., "Component masses and inclination of binary systems containing a pulsar, determined from relativistic effects", Sov. Astron. Lett., 1, 2-4, (1975). 2.1

[46] Bulik, T., and Belczynski, K., "Compact Object Mergers as Progenitors of Short Gamma-Ray Bursts", Baltic Astron., 13, 280-283, (2004). 2.3

[47] Büning, A., and Ritter, H., "Numerical stability of mass transfer driven by Roche lobe overflow in close binaries", Astron. Astrophys., 445, 647-652, (2006). Related online version (cited on 21 June 2006):

http://adsabs.harvard.edu/abs/2006A\&A...445. .647B. 7 
[48] Burgay, M., D’Amico, N., Possenti, A., Manchester, R.N., Lyne, A.G., Joshi, B.C., McLaughlin, M.A., Kramer, M., Sarkissian, J.M., Camilo, F., Kalogera, V., Kim, C., and Lorimer, D.R., "An increased estimate of the merger rate of double neutron stars from observations of a highly relativistic system", Nature, 426, 531-533, (2003). 1, 2.1, 2.2, 2.2, 5.2

[49] Burgay, M., Possenti, A., Manchester, R.N., Kramer, M., McLaughlin, M.A., Lorimer, D.R., Stairs, I.H., Joshi, B.C., Lyne, A.G., Camilo, F., D'Amico, N., Freire, P.C.C., Sarkissian, J.M., Hotan, A.W., and Hobbs, G.B., "Long-Term Variations in the Pulse Emission from PSR J0737-3039B", Astrophys. J. Lett., 624, L113-L116, (2005). Related online version (cited on 21 June 2006):

http://adsabs.harvard.edu/abs/2005ApJ...624L.113B. 3.4

[50] Burrows, D.N., Grupe, D., Capalbi, M., Panaitescu, A., Patel, S.K., Kouveliotou, C., Zhang, B., Mészáros, P., Chincarini, G., Gehrels, N., and Wijers, R.A.M., "Jet Breaks in Short Gamma-Ray Bursts. II: The Collimated Afterglow of GRB 051221A", Astrophys. J., submitted, (2006). Related online version (cited on 21 June 2006):

http://arXiv.org/abs/astro-ph/0604320. 3

[51] Camilo, F., and Rasio, F.A., "Pulsars in Globular Clusters", in Rasio, F.A., and Stairs, I.H., eds., Binary Radio Pulsars, Meeting at the Aspen Center for Physics, Colorado, 12 16 January 2004, vol. 328 of ASP Conference Series, 147-169, (Astronomical Society of the Pacific, San Francisco, U.S.A., 2005). Related online version (cited on 21 June 2006):

http://arXiv.org/abs/astro-ph/0501226. 2.2

[52] Canal, R., Méndez, J., and Ruiz-Lapuente, P., "Identification of the Companion Stars of Type Ia supernovae", Astrophys. J. Lett., 550, L53-L56, (2001). Related online version (cited on 21 June 2006):

http://adsabs.harvard.edu/abs/2001ApJ ...550L . .53C. 7

[53] Cappellaro, E., and Turatto, M., "Supernova Types and Rates", in Vanbeveren, D., ed., The Influence of Binaries on Stellar Population Studies, Conference in Brussels, Belgium, 21 25 August 2000, vol. 264 of Astrophysics and Space Science Library, 199, (Kluwer Academic Publishers, Dordrecht, Netherlands; Boston, U.S.A., 2001). Related online version (cited on 21 June 2006):

http://adsabs.harvard.edu/abs/2001ibsp.conf . .199C. 7

[54] Cassisi, S., Iben Jr, I., and Tornambè, A., "Hydrogen-Accreting Carbon-Oxygen White Dwarfs", Astrophys. J., 496, 376-385, (1998). 7

[55] Chevalier, R.A., "Neutron star accretion in a stellar envelope", Astrophys. J. Lett., 411, L33-L36, (1993). Related online version (cited on 4 November 2006):

http://adsabs.harvard.edu/abs/1993ApJ ...411L. .33C. 6

[56] Chugai, N.N., "Pulsar Space Velocities and Neutrino Chirality", Sov. Astron. Lett., 10, 87, (1984). 3.4

[57] Chugai, N.N., and Yungelson, L.R., "Type Ia supernovae in dense circumstellar gas", Astron. Lett., 30, 65-72, (2004). Related online version (cited on 10 November 2006):

http://arXiv.org/abs/astro-ph/0308297. 13

[58] Clark, J.P.A., and Eardley, D.M., "Evolution of close neutron star binaries", Astrophys. J., 215, 311-322, (1977). 1 
[59] Clark, J.P.A., van den Heuvel, E.P.J., and Sutantyo, W., "Formation of neutron star binaries and their importance for gravitational radiation", Astron. Astrophys., 72, 120-128, (1979). 1

[60] Colgate, S.A., "Neutron-Star Formation, Thermonuclear Supernovae, and Heavy-Element Reimplosion", Astrophys. J., 163, 221, (1971). Related online version (cited on 4 November 2006):

http://adsabs.harvard.edu/abs/1971ApJ...163..221C. 6

[61] Cooray, A., "Gravitational-wave background of neutron star-white dwarf binaries", Mon. Not. R. Astron. Soc., 354, 25-30, (2004). Related online version (cited on 21 June 2006): http: //adsabs.harvard.edu/abs/2004MNRAS.354_..25C. 10

[62] Cooray, A., Farmer, A.J., and Seto, N., "The Optical Identification of Close White Dwarf Binaries in the Laser Interferometer Space Antenna Era", Astrophys. J. Lett., 601, L47-L50, (2004). Related online version (cited on 21 June 2006): http://adsabs.harvard.edu/abs/2004ApJ . .601L . .47C. 10

[63] Cornish, N.J., and Larson, S.L., "LISA data analysis: Source identification and subtraction", Phys. Rev. D, 67, 103001, (2003). Related online version (cited on 21 June 2006): http://adsabs.harvard.edu/abs/2003PhRvD..67j3001C. 12.2

[64] Corongiu, A., Kramer, M., Stappers, B.W., Lyne, A.G., Jessner, A., Possenti, A., D’Amico, N., and Löhmer, O., "The binary pulsar PSR J1811-1736: evidence of a low amplitude supernova kick", Astron. Astrophys., accepted, (2006). Related online version (cited on 20 November 2006):

http://arXiv.org/abs/astro-ph/0611436. 3.4

[65] Counselman, C.C., "Outcomes of Tidal Evolution", Astrophys. J., 180, 307-316, (1973). 3.5

[66] Covino, S., Malesani, D., Israel, G.L., D’Avanzo, P., Antonelli, L.A., Chincarini, G., Fugazza, D., Conciatore, M.L., Della Valle, M., Fiore, F., Guetta, D., Hurley, K., Lazzati, D., Stella, L., Tagliaferri, G., Vietri, M., Campana, S., Burrows, D.N., D’Elia, V., Filliatre, P., Gehrels, N., Goldoni, P., Melandri, A., Mereghetti, S., Mirabel, I.F., Moretti, A., Nousek, J., O’Brien, P.T., Pellizza, L.J., Perna, R., Piranomonte, S., Romano, P., and Zerbi, F.M., "Optical emission from GRB 050709: A short/hard GRB in a star-forming galaxy", Astron. Astrophys. 447, L5-L8, (2006). Related online version (cited on 21 June 2006): http://adsabs.harvard.edu/abs/2006A\&A. . 447L . .5C. 2.3

[67] Cox, J.P., and Giuli, R.T., Principles of Stellar Structure, (Gordon and Breach, New York, U.S.A., 1968). 1

[68] Crowder, J., and Cornish, N.J., "Beyond LISA: Exploring future gravitational wave missions", Phys. Rev. D, 72, 083005, (2005). Related online version (cited on 21 June 2006): http://arXiv.org/abs/gr-qc/0506015. 1

[69] Crowther, P.A, "Physical Properties of Wolf-Rayet Stars", Annu. Rev. Astron. Astrophys., submitted, (2006). Related online version (cited on 4 November 2006):

http://arXiv.org/abs/astro-ph/0610356. 4.2

[70] Cutler, C., and Thorne, K.S., "An Overview of Gravitational-Wave Sources", in Bishop, N.T., and Maharaj, S.D., eds., General Relativity and Gravitation, Proceedings of the 16th International Conference on General Relativity and Gravitation, Durban, South Africa, 1521 July 2001, 72-111, (World Scientific, Singapore; River Edge, U.S.A., 2002). Related online 
version (cited on 4 November 2006):

http://arXiv.org/abs/gr-qc/0204090. 6.2

[71] de Freitas Pacheco, J.A., Regimbau, T., Vincent, S., and Spallicci, A., "Expected Coalescence Rates of Ns-Ns Binaries for Laser Beam Interferometers", Int. J. Mod. Phys. D, 15, 235-249, (2006). Related online version (cited on 21 June 2006):

http://adsabs.harvard.edu/abs/2006IJMPD. .15 . 235D. 5.2

[72] de Kool, M., "Common envelope evolution and double cores of planetary nebulae", Astrophys. J., 358, 189-195, (1990). 3.5

[73] de Marco, O., and Moe, M., "Common Envelope Evolution through Planetary Nebula Eyes", in Szczerba, R., Stasińska, G., and Górny, S.K., eds., Planetary Nebulae as Astronomical Tools, International Conference in Gdańsk, Poland, 28 June - 2 July 2005, vol. 804 of AIP Conference Proceedings, 169-172, (American Institute of Physics, Melville, U.S.A., 2005). Related online version (cited on 21 June 2006):

http://adsabs.harvard.edu/abs/2005AIPC. . 804 . 169D. 3.5

[74] Delgado-Donate, E.J., Clarke, C.J., and Bate, M.R.and Hodgkin, S.T., "On the properties of young multiple stars", Mon. Not. R. Astron. Soc., 351, 617-629, (2004). 1

[75] Deloye, C.J., and Bildsten, L., "White Dwarf Donors in Ultracompact Binaries: The Stellar Structure of Finite-Entropy Objects", Astrophys. J., 598, 1217-1228, (2003). Related online version (cited on 21 June 2006):

http://adsabs.harvard.edu/abs/2003ApJ ...598.1217D. 9, 12.1

[76] Deloye, C.J., Bildsten, L., and Nelemans, G., "Arbitrarily Degenerate Helium White Dwarfs as Donors in AM Canum Venaticorum Binaries", Astrophys. J., 624, 934-945, (2005). Related online version (cited on 21 June 2006):

http://adsabs.harvard.edu/abs/2005ApJ...624 . 934D. 12.1, 18

[77] Dessart, L., Burrows, A., Ott, C., Livne, E., Yoon, S.-C., and Langer, N., "Multi-Dimensional Simulations of the Accretion-Induced Collapse of White Dwarfs to Neutron Stars", Astrophys. $J ., 644,1063-1084,(2006)$. Related online version (cited on 21 June 2006):

http://adsabs.harvard.edu/abs/2006astro.ph..1603D. 1, 3.4

[78] Dewey, R.J., and Cordes, J.M., "Monte Carlo simulations of radio pulsars and their progenitors", Astrophys. J., 321, 780-798, (1987). 5.2

[79] Dewi, J.D.M., and Pols, O.R., "The late stages of evolution of helium star-neutron star binaries and the formation of double neutron star systems", Mon. Not. R. Astron. Soc., 344, 629-643, (2003). Related online version (cited on 20 November 2006):

http://arXiv.org/abs/astro-ph/0306066. 3.4

[80] Dewi, J.D.M., and Tauris, T.M., "On the energy equation and efficiency parameter of the common envelope evolution", Astron. Astrophys., 360, 1043-1051, (2000). 3.5

[81] Dewi, J.D.M., and van den Heuvel, E.P.J., "The formation of the double neutron star pulsar J0737-3039", Mon. Not. R. Astron. Soc., 349, 169-172, (2004). 4.1

[82] Di Stefano, R., and Rappaport, S., "How Large is the Population of Luminous Supersoft X-Ray Sources?", in Fruchter, A.S., Tavani, M., and Backer, D.C., eds., Millisecond Pulsars: A Decade of Surprise, Conference in Aspen, Colorado, 3 - 7 January 1994, vol. 72 of ASP Conference Series, 155, (Astronomical Society of the Pacific, San Francisco, U.S.A., 1995). 7 
[83] Dorofeev, O.F., Rodionov, V.N., and Ternov, I.M., "Anisotropic Neutrino Emission from Beta-Decays in a Strong Magnetic Field", Sov. Astron. Lett., 11, 123, (1985). 3.4

[84] Downes, R.A., Webbink, R.F., Shara, M.M., Ritter, H., Kolb, U., and Duerbeck, H.W., "Catalog of Cataclysmic Variables (Downes+ 2001-2006)", web interface to database, HarvardSmithsonian Center for Astrophysics. URL (cited on 21 June 2006): http://vizier.cfa.harvard.edu/viz-bin/VizieR?-source=V/123A. 8

[85] Drake, J.J., and Sarna, M.J., "X-Ray Evidence of the Common Envelope Phase of V471 Tauri", Astrophys. J. Lett., 594, L55-L58, (2003). 3.5

[86] Duquennoy, A., and Mayor, M., "Multiplicity among solar-type stars in the solar neighbourhood. II - Distribution of the orbital elements in an unbiased sample", Astron. Astrophys., 248, 485-524, (1991). 1

[87] Eck, C.R., Cowan, J.J., Roberts, D.A., Boffi, F.R., and Branch, D., "Radio Observations of the Type Ia Supernova 1986G as a Test of a Symbiotic-Star Progenitor", Astrophys. J. Lett., 451, L53, (1995). Related online version (cited on 21 June 2006): http://adsabs.harvard.edu/abs/1995ApJ . . 451L . 53E. 7

[88] Edlund, J.A., Tinto, M., Królak, A., and Nelemans, G., "White-dwarf-white-dwarf galactic background in the LISA data", Phys. Rev. D, 71, 1-16, 122003, (2005). Related online version (cited on 21 June 2006): http://adsabs.harvard.edu/abs/2005PhRvD..7112003E. 12.2

[89] Eggleton, P.P., "Approximations to the radii of Roche lobes", Astrophys. J., 268, 368-369, (1983). 3.5

[90] Eichler, D., Livio, M., Piran, T., and Schramm, D.N., "Nucleosynthesis, Neutrino Bursts and $\gamma$-Rays from Coalescing Neutron Stars", Nature, 340, 126-128, (1989). 2.3

[91] European Space Agency, "GAIA homepage", project homepage, (2006). URL (cited on 21 June 2006): http://sci.esa.int/science-e/www/area/index.cfm?fareaid=26. 12.2

[92] Evans, C.R., Iben Jr, I., and Smarr, L.L., "Degenerate dwarf binaries as promising, detectable sources of gravitational radiation", Astrophys. J., 323, 129-139, (1987). Related online version (cited on 21 June 2006):

http://adsabs.harvard.edu/abs/1987ApJ . . 323. .129E. 10

[93] Fabian, A.C., Pringle, J.E., and Rees, M.J., "Tidal capture formation of binary systems and X-ray sources in globular clusters", Mon. Not. R. Astron. Soc., 172, 15-18, (1975). Related online version (cited on 21 June 2006):

http://adsabs.harvard.edu/abs/1975MNRAS.172P..15F. 7

[94] Fadeyev, Y.A., and Novikova, M.F., "Radial Pulsations of Helium Stars with Masses from 1 to $10 M_{\odot} "$, Astron. Lett., 29, 522-529, (2003). Related online version (cited on 21 June 2006): http://adsabs.harvard.edu/abs/2003AstL...29. .522F. 2

[95] Fadeyev, Y.A., and Novikova, M.F., "Radial Pulsations of Helium Stars with Masses from 10 to $50 M_{\odot} "$, Astron. Lett., 30, 707-714, (2004). Related online version (cited on 21 June 2006):

http://adsabs.harvard.edu/abs/2004AstL...30..707F. 2 
[96] Faller, J.E., Bender, P.L., Hall, J.L., Hils, D., Stebbins, R.T., and Vincent, M.A., "An antenna for laser gravitational-wave observations in space", Adv. Space Res., 9, 107-111, (1989). Related online version (cited on 21 June 2006):

http://adsabs.harvard.edu/abs/1989AdSpR...9..107F. 1, 10

[97] Faulkner, A.J., Kramer, M., Lyne, A.G., Manchester, R.N., McLaughlin, M.A., Stairs, I.H., Hobbs, G., Possenti, A., Lorimer, D.R., D'Amico, N., Camilo, F., and Burgay, M., "PSR J1756-2251: A New Relativistic Double Neutron Star System", Astrophys. J. Lett., 618, L119-L122, (2005). 2.1

[98] Faulkner, A.J., Stairs, I.H., Kramer, M., Lyne, A.G., Hobbs, G., Possenti, A., Lorimer, D.R., Manchester, R.N., McLaughlin, M.A., D'Amico, N., Camilo, F., and Burgay, M., "The Parkes Multibeam Pulsar Survey - V. Finding binary and millisecond pulsars", Mon. Not. R. Astron. Soc., 355, 147-158, (2004). 1

[99] Faulkner, J., "Ultrashort-Period Binaries, Gravitational Radiation, and Mass Transfer. I. The Standard Model, with Applications to WZ Sagittae and Z Camelopardalis", Astrophys. J., 170, L99, (1971). Related online version (cited on 21 June 2006):

http://adsabs.harvard.edu/abs/1971ApJ...170L. .99F. 9

[100] Faulkner, J., Flannery, B.P., and Warner, B., "Ultrashort-period binaries. II. HZ 29 (= AM CVn): A double-white-dwarf semidetached postcataclysmic nova?", Astrophys. J., 175, L79, (1972). 7

[101] Fedorova, A.V., and Ergma, E.V., "Evolution of binaries with ultra-short periods - Systematic study", Astrophys. J. Suppl. Ser., 151, 125-134, (1989). Related online version (cited on 21 June 2006):

http://adsabs.harvard.edu/abs/1989Ap\&SS.151. .125F. 7

[102] Fedorova, A.V., Tutukov, A.V., and Yungelson, L.R., "Type Ia Supernovae in semi-detached binary systems", Astron. Lett., 30, 73-85, (2004). Related online version (cited on 21 June 2006):

http://arXiv.org/abs/astro-ph/0309052. 7, 7, 7

[103] Festin, L, "The luminosity function of white dwarfs and $M$ dwarfs using dark nebulae as opaque outer screens", Astron. Astrophys., 336, 883-894, (1998). 12.2

[104] Figer, D.F., "An upper limit to the masses of stars", Nature, 434, 192-194, (2005). Related online version (cited on 21 June 2006):

http://adsabs.harvard.edu/abs/2005Natur.434..192F. 1

[105] Flanagan, É.É., and Hughes, S.A., "Measuring gravitational waves from binary black hole coalescences. I. Signal to noise for inspiral, merger, and ringdown", Phys. Rev. D, 57, 45354565, (1998). 6.1

[106] Flannery, B.P., and van den Heuvel, E.P.J., "On the origin of the binary pulsar PSR 1913+16", Astron. Astrophys., 39, 61-67, (1975). 3.3, 4.1

[107] Förster, F., Wolf, C., Podsiadlowski, P., and Han, Z., "Constraints on Type Ia supernova progenitor time delays from high- $z$ supernovae and the star formation history", Mon. Not. R. Astron. Soc., 368, 1893-1904, (2006). Related online version (cited on 21 June 2006): http://adsabs.harvard.edu/abs/2006MNRAS.368.1893F. 1 
[108] Foss, D., Wade, R.A., and Green, R.F., "Limits on the space density of double degenerates as type Ia supernova progenitors", Astrophys. J., 374, 281-287, (1991). Related online version (cited on 21 June 2006):

http://adsabs.harvard.edu/abs/1991ApJ...374..281F. 8

[109] Fox, D.B., Frail, D.A., Price, P.A., Kulkarni, S.R., Berger, E., Piran, T., Soderberg, A.M., Cenko, S.B., Cameron, P.B., Gal-Yam, A., Kasliwal, M.M., Moon, D.-S., Harrison, F.A., Nakar, E., Schmidt, B.P., Penprase, B., Chevalier, R.A., Kumar, P., Roth, K., Watson, D., Lee, B.L., Shectman, S., Phillips, M.M., Roth, M., McCarthy, P.J., Rauch, M., Cowie, L., Peterson, B.A., Rich, J., Kawai, N., Aoki, K., Kosugi, G., Totani, T., Park, H.-S., MacFadyen, A., and Hurley, K.C., "The afterglow of GRB 050709 and the nature of the short-hard $\gamma$-ray bursts", Nature, 437, 845-850, (2005). Related online version (cited on 21 June 2006):

http://adsabs.harvard.edu/abs/2005Natur.437..845F. 2.3

[110] Fryer, C.L., "Mass Limits For Black Hole Formation", Astrophys. J., 522, 413-418, (1999). 4.2

[111] Fryer, C.L., ed., Stellar Collapse, Proceedings of "Core Collapse of Massive Stars", 200th AAS meeting, Albuquerque, NM, June 2002, vol. 302 of Astrophysics and Space Science Library, (Kluwer Academic Publishers, Dordrecht, Netherlands; Boston, U.S.A., 2004). 1, 4.2

[112] Fryer, C.L., Burrows, A., and Benz, W., "Population Syntheses for Neutron Star Systems with Intrinsic Kicks", Astrophys. J., 496, 333, (1998). URL (cited on 4 November 2006): http://adsabs.harvard.edu/abs/1998ApJ . . 496 . 333F. 3.4

[113] Fryer, C.L., and Kalogera, V., "Theoretical Black Hole Mass Distributions", Astrophys. J., 554, 548-560, (2001). 4.2

[114] Fukugita, M., and Peebles, P.J.E., "The Cosmic Energy Inventory", Astrophys. J., 616, 643668, (2004). Related online version (cited on 21 June 2006):

http://arXiv.org/abs/gr-qc/0406095. 1

[115] García-Berro, E., Ritossa, C., and Iben Jr, I., "On the Evolution of Stars That Form ElectronDegenerate Cores Processed by Carbon Burning. III. The Inward Propagation of a Carbonburning Flame and Other Properties of a $9 M_{\odot}$ Model Star", Astrophys. J., 485, 765, (1997). Related online version (cited on 21 June 2006):

http://adsabs.harvard.edu/abs/1997ApJ . .485. .765G. 1

[116] Gehrels, N., Sarazin, C.L., O’Brien, P.T., Zhang, B., Barbier, L., Barthelmy, S.D., Blustin, A., Burrows, D.N., Cannizzo, J., Cummings, J.R., Goad, M., Holland, S.T., Hurkett, C.P., Kennea, J.A., Levan, A., Markwardt, C.B., Mason, K.O., Mészáros, P., Page, M., Palmer, D.M., Rol, E., Sakamoto, T., Willingale, R., Angelini, L., Beardmore, A., Boyd, P.T., Breeveld, A., Campana, S., Chester, M.M., Chincarini, G., Cominsky, L.R., Cusumano, G., de Pasquale, M., Fenimore, E.E., Giommi, P., Gronwall, C., Grupe, D., Hill, J.E., Hinshaw, D., Hjorth, J., Hullinger, D., Hurley, K.C., Klose, S., Kobayashi, S., Kouveliotou, C., Krimm, H.A., Mangano, V., Marshall, F.E., McGowan, K., Moretti, A., Mushotzky, R.F., Nakazawa, K., Norris, J.P., Nousek, J.A., Osborne, J.P., Page, K., Parsons, A.M., Patel, S., Perri, M., Poole, T., Romano, P., Roming, P.W.A., Rosen, S., Sato, G., Schady, P., Smale, A.P., Sollerman, J., Starling, R., Still, M., Suzuki, M., Tagliaferri, G., Takahashi, T., Tashiro, M., Tueller, J., Wells, A.A., White, N.E., and Wijers, R.A.M.J., "A short $\gamma$-ray burst apparently associated with an elliptical galaxy at redshift $z=0.225 "$, Nature, 437,

Living Reviews in Relativity

http://www. livingreviews.org/Irr-2006-6 
851-854, (2005). Related online version (cited on 21 June 2006):

http://adsabs.harvard.edu/abs/2005Natur.437..851G. 2.3, 13

[117] Ghosh, K.K., Rappaport, S., Tennant, A.F., Swartz, D.A., Pooley, D., and Madhusudhan, N., "Discovery of a $3.6 \mathrm{hr}$ Eclipsing Luminous X-Ray Binary in the Galaxy NGC 4214", Astrophys. J., 650, 872-878, (2006). Related online version (cited on 21 June 2006): http://arXiv.org/abs/astro-ph/0604466. 3.2.3

[118] Giacconi, R., Murray, S., Gursky, H., Kellogg, E., Schreier, E., Matilsky, T., Koch, D., and Tananbaum, H., "The Third UHURU Catalog of X-Ray Sources", Astrophys. J. Suppl. Ser., 27, 37, (1974). Related online version (cited on 21 June 2006): http: //adsabs.harvard.edu/abs/1974ApJS . . 27 . .37G. 8

[119] Gil-Pons, P., García-Berro, E., José, J., Hernanz, M., and Truran, J.W., "The frequency of occurrence of novae hosting an ONe white dwarf", Astron. Astrophys., 407, 1021-1028, (2003). Related online version (cited on 21 June 2006): http://adsabs.harvard.edu/abs/2003A\&A. . 407.1021G. 1

[120] Gokhale, V., Meng Peng, X., and Frank, J., "Evolution of Close White Dwarf Binaries", (October, 2006). URL (cited on 4 November 2006): http://arXiv.org/abs/astro-ph/0610919. 7, 9

[121] Grindlay, J., Portegies Zwart, S.F., and McMillan, S.L.W., "Short gamma-ray bursts from binary neutron star mergers in globular clusters", Nature Phys., 2, 116-119, (2006). Related online version (cited on 21 June 2006):

http://adsabs.harvard.edu/abs/2006NatPh...2..116G. 2.3

[122] Grishchuk, L.P., Lipunov, V.M., Postnov, K.A., Prokhorov, M.E., and Sathyaprakash, B.S., "Gravitational Wave Astronomy: In Anticipation of First Sources to be Detected", Phys. Usp., 44, 1-51, (2001). Related online version (cited on 21 June 2006): http://arXiv.org/abs/astro-ph/0008481. 1, 6, 6.1, 10, 13

[123] Groot, P.J., "OmegaWhite and VPHAS+", other, Astro-Wise, (2006). URL (cited on 21 June 2006):

http://www . astro-wise.org/Presentations/LC2005course/astrowiseleiden_groot. pdf. 12.2

[124] Guerrero, J., García-Berro, E., and Isern, J., "Smoothed Particle Hydrodynamics simulations of merging white dwarfs", Astron. Astrophys., 413, 257-272, (2004). 7

[125] Gunn, J.E., and Ostriker, J.P., "On the Nature of Pulsars. III. Analysis of Observations", Astrophys. J., 160, 979-1002, (1970). 3.4

[126] Hachisu, I., and Kato, M., "Recurrent Novae as a Progenitor System of Type Ia Supernovae. I. RS Ophiuchi Subclass: Systems with a Red Giant Companion", Astrophys. J., 558, 323350, (2001). Related online version (cited on 21 June 2006): http://adsabs.harvard.edu/abs/2001ApJ ...558. .323H. 7

[127] Hachisu, I., Kato, M., Nomoto, K., and Umeda, H., "A New Evolutionary Path to Type Ia Supernovae: A Helium-rich Supersoft X-Ray Source Channel", Astrophys. J., 519, 314-323, (1999). Related online version (cited on 21 June 2006):

http: //adsabs.harvard.edu/abs/1999ApJ . . 519. .314H. 7 
[128] Halbwachs, J.L., Mayor, M., Udry, S., and Arenou, F., "Multiplicity among solar-type stars. III. Statistical properties of the F7-K binaries with periods up to 10 years", Astron. Astrophys., 397, 159-175, (2003). 1

[129] Hamann, W.-R., Gräfener, G., and Liermann, A., "The Galactic WN stars. Spectral analyses with line-blanketed model atmospheres versus stellar evolution models with and without rotation", Astron. Astrophys., 457, 1015-1031, (2006). Related online version (cited on 4 November 2006):

http://adsabs.harvard.edu/abs/2006A\&A...457.1015H. 4.2

[130] Hamuy, M., Phillips, M.M., Suntzeff, N.B., Maza, J., Gonzalez, L.E., Roth, M., Krisciunas, K., Morrell, N., Green, E.M., Persson, S.E., and McCarthy, P.E., "An asymptotic-giantbranch star in the progenitor system of a type Ia supernova", Nature, 424, 651-654, (2003). Related online version (cited on 21 June 2006): http://adsabs.harvard.edu/abs/2003Natur.424..651H. 13

[131] Han, Z., "The formation of double degenerates and related objects", Mon. Not. R. Astron. Soc., 296, 1019-1040, (1998). Related online version (cited on 21 June 2006): http://adsabs.harvard.edu/abs/1998MNRAS . 296.1019H. 10

[132] Han, Z., and Podsiadlowski, P., "The single-degenerate channel for the progenitors of Type Ia supernovae", Mon. Not. R. Astron. Soc., 350, 1301-1309, (2004). Related online version (cited on 21 June 2006): http://adsabs.harvard.edu/abs/2004MNRAS.350.1301H. 7, 7

[133] Han, Z., and Podsiadlowski, P., "A single-degenerate model for the progenitor of the Type Ia supernova 2002ic", Mon. Not. R. Astron. Soc., 368, 1095-1100, (2006). Related online version (cited on 21 June 2006):

http://adsabs.harvard.edu/abs/2006MNRAS.368.1095H. 7

[134] Han, Z., Podsiadlowski, P., Maxted, P.F.L., Marsh, T.R., and Ivanova, N., "The origin of subdwarf B stars - I. The formation channels", Mon. Not. R. Astron. Soc., 336, 449-466, (2002). 3.5

[135] Han, Z., Podsiadlowski, P., Maxted, P.F.L., Marsh, T.R., and Ivanova, N., "The origin of subdwarf B stars - I. The formation channels", Mon. Not. R. Astron. Soc., 336, 449-466, (2002). Related online version (cited on 21 June 2006): http://adsabs.harvard.edu/abs/2002MNRAS.336. .449H. 7

[136] Han, Z., and Webbink, R.F., "Stability and energetics of mass transfer in double white dwarfs", Astron. Astrophys., 349, L17-L20, (1999). 9

[137] Hansen, B.M.S., "Cooling models for old white dwarfs", Astrophys. J., 520, 680-695, (1999). 11

[138] Heger, A., Fryer, C.L., Woosley, S.E., Langer, N., and Hartmann, D.H., "How Massive Single Stars End Their Life", Astrophys. J., 591, 288-300, (2003). Related online version (cited on 4 November 2006):

http://adsabs.harvard.edu/abs/2003ApJ...591..288H. 4.2

[139] Hellings, R.W., "LISA data analysis: The detection and initial guess problems for monochromatic binaries", Class. Quantum Grav., 20, 1019-1029, (2003). Related online version (cited on 21 June 2006):

http://adsabs.harvard.edu/abs/2003CQGra..20.1019H. 11 
[140] Hills, J.G., "The effects of sudden mass loss and a random kick velocity produced in a supernova explosion on the dynamics of a binary star of arbitrary orbital eccentricity Applications to X-ray binaries and to the binary pulsars", Astrophys. J., 267, 322-333, (1983). URL (cited on 4 November 2006):

http://adsabs.harvard.edu/abs/1983ApJ . . 267 . .322H. 3.3

[141] Hils, D., "Confusion Noise Estimate for Gravitational Wave Measurements in Space", in Folkner, W.M., ed., Laser Interferometer Space Antenna, Second International LISA Symposium on the Detection and Observation of Gravitational Waves in Space, Pasadena, California, July 1998, vol. 456 of AIP Conference Proceedings, 68-78, (American Institure of Physics, Woodbury, U.S.A., 1998). Related online version (cited on 21 June 2006): http://adsabs.harvard.edu/abs/1998AIPC . 456 ..68H. 10

[142] Hils, D., and Bender, P.L., "Gravitational Radiation from Helium Cataclysmics", Astrophys. J., 537, 334-341, (2000). Related online version (cited on 21 June 2006):

http://adsabs.harvard.edu/abs/2000ApJ . .537 . .334H. 10, 10, 11

[143] Hils, D, Bender, P.L., and Webbink, R.F., "Gravitational radiation from the Galaxy", Astrophys. J., 360, 75-94, (1990). 10

[144] Hjellming, M.S., and Webbink, R.F., "Thresholds for rapid mass transfer in binary systems. I. Polytropic models", Astrophys. J., 318, 794-808, (1987). 7

[145] Hjorth, J., Sollerman, J., Møller, P., Fynbo, J.P.U., Woosley, S.E., Kouveliotou, C., Tanvir, N.R., Greiner, J., Andersen, M.I., Castro-Tirado, A.J., Castro Cerón, J.M., Fruchter, A.S., Gorosabel, J.and Jakobsson, P., Kaper, L., Klose, S., Masetti, N.and Pedersen, H., Pedersen, K., Pian, E., Palazzi, E., Rhoads, J.E., Rol, E., van den Heuvel, E.P.J., Vreeswijk, P.M., Watson, D., and Wijers, R.A.M.J., "A very energetic supernova associated with the $\gamma$-ray burst of 29 March 2003", Nature, 423, 847-850, (2003). 2.3

[146] Hjorth, J., Watson, D., Fynbo, J.P.U., Price, P.A., Jensen, B.L., Jørgensen, U.G., Kubas, D., Gorosabel, J., Jakobsson, P., Sollerman, J., Pedersen, K., and Kouveliotou, C., "The optical afterglow of the short $\gamma$-ray burst GRB 050709", Nature, 437, 859-861, (2005). Related online version (cited on 21 June 2006):

http://adsabs.harvard.edu/abs/2005Natur.437. . 859H. 2.3

[147] Hobbs, G., Lorimer, D.R., Lyne, A.G., and Kramer, M., "A statistical study of 233 pulsar proper motions", Mon. Not. R. Astron. Soc., 360, 974-992, (2005). Related online version (cited on 21 June 2006):

http://arXiv.org/abs/astro-ph/0504584. 3.4

[148] Höflich, P., Khokhlov, A., Wheeler, J.C., Phillips, M.M., Suntzeff, N.B., and Hamuy, M., "Maximum Brightness and Postmaximum Decline of Light Curves of Type Ia Supernovae: A Comparison of Theory and Observations", Astrophys. J. Lett., 472, L81-L84, (1996). Related online version (cited on 21 June 2006):

http://adsabs.harvard.edu/abs/1996ApJ...472L. .81H. 7

[149] Holberg, J.B., Oswalt, T.D., and Sion, E.M., "A Determination of the Local Density of White Dwarf Stars", Astrophys. J., 571, 512-518, (2002). Related online version (cited on 21 June 2006):

http://adsabs.harvard.edu/abs/2002ApJ . .571. .512H. 12.2

[150] Hopman, C., Guetta, D., Waxman, E., and Portegies Zwart, S., "The Redshift Distribution of Short Gamma-Ray Bursts from Dynamically Formed Neutron Star Binaries", Astrophys. 
J. Lett., 643, L91-L94, (2006). Related online version (cited on 21 June 2006):

http://adsabs.harvard.edu/abs/2006ApJ...643L..91H. 2.3

[151] Hotan, A.W., Bailes, M., and Ord, S.M., "Geodetic Precession in PSR J1141-6545", Astrophys. J., 624, 906-913, (2005). Related online version (cited on 21 June 2006): http://adsabs.harvard.edu/abs/2005ApJ . .624. .906H. 3.4

[152] Howell, D.A., Sullivan, M., Nugent, P.E., Ellis, R.S., Conley, A.J., Le Borgne, D., Carlberg, R.G., Guy, J., Balam, D., Basa, S., Fouchez, D., Hook, I.M., Hsiao, E.Y., Neill, J.D., Pain, R., Perrett, K.M., and Pritchet, C.J., "The type Ia supernova SNLS-03D3bb from a superChandrasekhar-mass white dwarf star", Nature, 443, 308-311, (2006). Related online version (cited on 4 November 2006):

http://adsabs .harvard.edu/abs/2006Natur.443. .308H. 11, 7

[153] Hulse, R.A., and Taylor, J.H., "Discovery of a pulsar in a binary system", Astrophys. J. Lett., 195, L51-L53, (1975). 2.1

[154] Hurley, J.R., Tout, C.A., and Pols, O.R., "Evolution of binary stars and the effect of tides on binary populations", Mon. Not. R. Astron. Soc., 329, 897-928, (2002). Related online version (cited on 21 June 2006):

http://adsabs.harvard.edu/abs/2002MNRAS.329. .897H. 5.2

[155] Hurley, J.R., Tout, C.A., and Pols, O.R., "Evolution of binary stars and the effect of tides on binary populations", Mon. Not. R. Astron. Soc., 329, 897-928, (2002). Related online version (cited on 21 June 2006): http: //adsabs .harvard.edu/abs/2002MNRAS. 329. .897H. 10

[156] Hyman, S.D., Lazio, T.J.W., Kassim, N.E., Ray, P.S., Markwardt, C.B., and Yusef-Zadeh, F., "A powerful bursting radio source towards the Galactic Centre", Nature, 434, 50-52, (2005). 2.2

[157] Iben Jr, I., "On the evolution of binary components which first fill their Roche lobes after the exhaustion of central helium", Astrophys. J., 304, 201-216, (1986). Related online version (cited on 21 June 2006):

http://adsabs.harvard.edu/abs/1986ApJ . . 304..201I. 1

[158] Iben Jr, I., and Livio, M., "Common envelopes in binary star evolution", Publ. Astron. Soc. Pac., 105, 1373-1406, (1993). Related online version (cited on 08 December 2006): http://adsabs .harvard.edu/abs/1993PASP. . 105.1373I. 3.5, 3.5

[159] Iben Jr, I., Ritossa, C., and García-Berro, E., "On the Evolution of Stars That Form ElectronDegenerate Cores Processed by Carbon Burning. IV. Outward Mixing during the Second Dredge-Up Phase and Other Properties of a 10.5M $\odot$ Model Star", Astrophys. J., 489, 772, (1997). Related online version (cited on 21 June 2006):

http://adsabs.harvard.edu/abs/1997ApJ . . 489. .772I. 1

[160] Iben Jr, I., and Tutukov, A.V., "Supernovae of type I as end products of the evolution of binaries with components of moderate initial mass $\left(M \lesssim 9 M_{\odot}\right)$ ", Astrophys. J. Suppl. Ser., 54, 335-372, (1984). Related online version (cited on 21 June 2006): http://adsabs.harvard.edu/abs/1984ApJS..54 . 335I. 7, 7, 8, 10

[161] Iben Jr, I., and Tutukov, A.V., "On the evolution of close binaries with components of initial mass between 3 solar masses and 12 solar masses", Astrophys. J. Suppl. Ser., 58, 661-710, (1985). 1, 7 
[162] Iben Jr, I., and Tutukov, A.V., "Helium star cataclysmics", Astrophys. J., 370, 615-629, (1991). Related online version (cited on 21 June 2006):

http://adsabs.harvard.edu/abs/1991ApJ...370..615I. 7

[163] Iben Jr, I., and Tutukov, A.V., "On the Evolution of Symbiotic Stars and Other Binaries with Accreting Degenerate Dwarfs", Astrophys. J. Suppl. Ser., 105, 145, (1996). Related online version (cited on 21 June 2006):

http://adsabs .harvard.edu/abs/1996ApJS . .105. .145I. 7

[164] Iben Jr, I., Tutukov, A.V., and Fedorova, A.V., "On the Luminosity of White Dwarfs in Close Binaries Merging under the Influence of Gravitational Wave Radiation", Astrophys. $J .$, 503, 344, (1998). Related online version (cited on 21 June 2006):

http://adsabs.harvard.edu/abs/1998ApJ...503. .344I. 12

[165] Iben Jr, I., Tutukov, A.V., and Yungelson, L.R., "A Model of the Galactic X-Ray Binary Population. II. Low-Mass X-Ray Binaries in the Galactic Disk", Astrophys. J. Suppl. Ser., 100, 233, (1995). 4.1

[166] in't Zand, J.J.M., Cumming, A., van der Sluys, M.V., Verbunt, F., and Pols, O.R., "On the possibility of a helium white dwarf donor in the presumed ultracompact binary 2S 0918549", Astron. Astrophys., 441, 675-684, (2005). Related online version (cited on 4 November 2006):

http://adsabs.harvard.edu/abs/2005A\&A...441..675I. 7

[167] Isern, J., Labay, J., Hernanz, M., and Canal, R., "Collapse and explosion of white dwarfs. I. Precollapse evolution", Astrophys. J., 273, 320-329, (1983). Related online version (cited on 21 June 2006):

http://adsabs.harvard.edu/abs/1983ApJ ...273. .320I. 7

[168] Israel, G.L., Panzera, M.R., Campana, S., Lazzati, D., Covino, S., Tagliaferri, G., and Stella, L., "The discovery of $321 \mathrm{~S}$ pulsations in the ROSAT HRI light curves of 1BMW J080622.8+152732 = RX J0806.3+1527", Astron. Astrophys., 349, L1-L4, (1999). Related online version (cited on 21 June 2006):

http://adsabs.harvard.edu/abs/1999A\&A...349L...1I. 11

[169] Ivanova, N., Belczynski, K., Kalogera, V., Rasio, F.A., and Taam, R.E., "The Role of Helium Stars in the Formation of Double Neutron Stars", Astrophys. J., 592, 475-485, (2003). 4.1

[170] Ivanova, N., Rasio, F.A., Lombardi, J.C., Dooley, K.L., and Proulx, Z.F., "Formation of Ultracompact X-Ray Binaries in Dense Star Clusters", Astrophys. J. Lett., 621, L109-L112, (2005). Related online version (cited on 21 June 2006):

http://adsabs.harvard.edu/abs/2005ApJ...621L.109I. 7

[171] Ivanova, N., and Taam, R.E., "Thermal Timescale Mass Transfer and the Evolution of White Dwarf Binaries", Astrophys. J., 601, 1058-1066, (2004). Related online version (cited on 21 June 2006):

http://adsabs.harvard.edu/abs/2004ApJ . .601.1058I. 7, 7

[172] Jeffery, D.J., Branch, D., and Baron, E., "On SN 2003fg: The Probable SuperChandrasekhar-Mass SN Ia", (September, 2006). URL (cited on 4 November 2006):

http://arXiv.org/abs/astro-ph/0609804. 11, 7

[173] Johnston, S., Hobbs, G., Vigeland, S., Kramer, M., Weisberg, J.M., and Lyne, A.G., "Evidence for alignment of the rotation and velocity vectors in pulsars", Mon. Not. R. Astron. 
Soc., 364, 1397-1412, (2005). Related online version (cited on 21 June 2006):

http://adsabs.harvard.edu/abs/2005MNRAS.364.1397J. 3.4

[174] Jonker, P.G., and Nelemans, G., "The distances to Galactic low-mass X-ray binaries: Consequences for black hole luminosities and kicks", Mon. Not. R. Astron. Soc., 354, 355-366, (2004). Related online version (cited on 21 June 2006):

http: //adsabs.harvard.edu/abs/2004MNRAS.354 . .355J. 3.4

[175] Kahabka, P., "Recurrent supersoft X-ray sources", Astron. Astrophys., 304, 227, (1995). Related online version (cited on 21 June 2006):

http://adsabs.harvard.edu/abs/1995A\&A...304..227K. 7

[176] Kahabka, P., "Super Soft Sources", in Lewin, W.H.G., and van der Klis, M., eds., Compact Stellar X-Ray Sources, Cambridge Astrophysics Series, (Cambridge University Press, Cambridge, U.K., 2006). Related online version (cited on 21 June 2006):

http://arXiv.org/abs/astro-ph/0212037. 7

[177] Kahabka, P., and van den Heuvel, P.J., "Luminous supersoft X-ray sources", Annu. Rev. Astron. Astrophys., 35, 69-100, (1997). 7

[178] Kalogera, V., "Orbital Characteristics of Binary Systems after Asymmetric Supernova Explosions", Astrophys. J., 471, 352, (1996). Related online version (cited on 4 November 2006): http://adsabs.harvard.edu/abs/1996ApJ . .471. .352K. 3.3

[179] Kalogera, V., "Spin-Orbit Misalignment in Close Binaries with Two Compact Objects", Astrophys. J., 541, 319-328, (2000). 4.2

[180] Kalogera, V., Kim, C., Lorimer, D.R., Burgay, M., D’Amico, N., Possenti, A., Manchester, R.N., Lyne, A.G., Joshi, B.C., McLaughlin, M.A., Kramer, M., Sarkissian, J.M., and Camilo, F., "The Cosmic Coalescence Rates for Double Neutron Star Binaries", Astrophys. J. Lett., 601, L179-L182, (2004). 1, 5.2

[181] Kalogera, V., Kim, C., Lorimer, D.R., Ihm, M., and Belczynski, K., "The Galactic Formation Rate of Eccentric Neutron Star - White Dwarf Binaries", in Rasio, F.A., and Stairs, I.H., eds., Binary Radio Pulsars, Meeting at the Aspen Center for Physics, Colorado, 12 - 16 January 2004, vol. 328 of ASP Conference Series, 261-267, (Astronomical Society of the Pacific, San Francisco, U.S.A., 2005). Related online version (cited on 21 June 2006): http://adsabs.harvard.edu/abs/2005ASPC..328. .261K. 7

[182] Kalogera, V., Narayan, R., Spergel, D.N., and Taylor, J.H., "The Coalescence Rate of Double Neutron Star Systems", Astrophys. J., 556, 340-356, (2001). 2.2

[183] Kalogera, V., and Webbink, R.F., "Formation of Low-Mass X-Ray Binaries. I. Constraints on Hydrogen-rich Donors at the Onset of the X-Ray Phase", Astrophys. J., 458, 301-311, (1996). 4.1

[184] Kalogera, V., and Webbink, R.F., "Formation of Low-Mass X-Ray Binaries. II. Common Envelope Evolution of Primordial Binaries with Extreme Mass Ratios", Astrophys. J., 493, 351-368, (1998). 4.1

[185] Karachentsev, I.D., Karachentseva, V.E., Huchtmeier, W.K., and Makarov, D.I., "A Catalog of Neighboring Galaxies", Astron. J., 127, 2031-2068, (2004). Related online version (cited on 4 November 2006):

http: //adsabs.harvard.edu/abs/2004AJ . . .127.2031K. 6.2 
[186] Kaspi, V.M., Bailes, M., Manchester, R.N., Stappers, B.W., and Bell, J.F., "Evidence from a precessing pulsar orbit for a neutron-star birth kick", Nature, 381, 584-586, (1996). 3.4

[187] Kato, M., and Hachisu, I., "Optically thick winds in nova outbursts", Astrophys. J., 437, 802-826, (1994). Related online version (cited on 21 June 2006): http://adsabs .harvard.edu/abs/1994ApJ . . 437 . .802K. 3.2 .3

[188] Katz, J.I., and Canel, L.M., "The Long and the Short of Gamma-Ray Bursts", Astrophys. J., 471, 915, (1996). 2.3

[189] Kim, C., Kalogera, V., and Lorimer, D.R., "The Probability Distribution of Binary Pulsar Coalescence Rates. I. Double Neutron Star Systems in the Galactic Field", Astrophys. J., 584, 985-995, (2003). 1, 2.2

[190] Kim, C., Kalogera, V., and Lorimer, D.R., "Effect of PSR J0737-3039 on the DNS Merger Rate and Implications for GW Detection", (August, 2006). URL (cited on 4 November 2006): http://arXiv.org/abs/astro-ph/0608280. 2.2, 2.2

[191] Kim, C., Kalogera, V., Lorimer, D.R., and White, T., "The Probability Distribution Of Binary Pulsar Coalescence Rates. II. Neutron Star-White Dwarf Binaries", Astrophys. J., 616, 1109-1117, (2004). Related online version (cited on 21 June 2006): http://adsabs.harvard.edu/abs/2004ApJ...616.1109K. 7

[192] Kitaura, F.S., Janka, H.-T., and Hillebrandt, W., "Explosions of O-Ne-Mg cores, the Crab supernova, and subluminous type II-P supernovae", Astron. Astrophys., 450, 345-350, (2006). Related online version (cited on 21 June 2006): http://adsabs.harvard.edu/abs/2006A\&A...450 . 345K. 3.4

[193] Knox, R.A., Hawkins, M.R.S., and Hambly, N.C., "A survey for cool white dwarfs and the age of the Galactic disc", Mon. Not. R. Astron. Soc., 306, 736-752, (1999). 12.2

[194] Kopal, Z., Close Binary Systems, vol. 5 of The International Astrophysics Series, (Chapman \& Hall; Wiley, London, U.K.; New York, U.S.A., 1959). 9

[195] Kornilov, V.G., and Lipunov, V.M., "Neutron Stars in Massive Binary Systems - Part One - Classification and Evolution", Sov. Astron., 27, 163, (1983). 5.2

[196] Kornilov, V.G., and Lipunov, V.M., "Neutron Stars in Massive Binary Systems - Part Two - Numerical Modeling", Sov. Astron., 27, 334, (1983). 5.2

[197] Kotake, K., Sato, K., and Takahashi, K., "Explosion mechanism, neutrino burst and gravitational wave in core-collapse supernovae", Rep. Prog. Phys., 69, 971-1143, (2006). Related online version (cited on 21 June 2006): http://adsabs.harvard.edu/abs/2006RPPh...69..971K. 1

[198] Kraft, R.P., Mathews, J., and Greenstein, J.L., "Binary Stars among Cataclysmic Variables. II. Nova WZ Sagittae: A Possible Radiator of Gravitational Waves", Astrophys. J., 136, 312, (1962). Related online version (cited on 21 June 2006): http://adsabs.harvard.edu/abs/1962ApJ ...136. .312K. 7

[199] Kroupa, P., Tout, C.A., and Gilmore, G., "The distribution of low-mass stars in the Galactic disc", Mon. Not. R. Astron. Soc., 262, 545-587, (1993). Related online version (cited on 4 November 2006):

http://adsabs.harvard.edu/abs/1993MNRAS.262. .545K. 1 
[200] Kroupa, P., and Weidner, C., "Galactic-Field Initial Mass Functions of Massive Stars", Astrophys. J., 598, 1076-1078, (2003). Related online version (cited on 4 November 2006): http://adsabs.harvard.edu/abs/2003ApJ...598.1076K. 1

[201] Kudritzki, R.-P., and Urbaneja, M.A., "Parameters and Winds of Hot Massive Stars", (July, 2006). URL (cited on 4 November 2006): http://arXiv.org/abs/astro-ph/0607460. 4.2

[202] Kuiper, G.P., "Problems of Double-Star Astronomy. I", Publ. Astron. Soc. Pac., 47, 15-42, (1935). Related online version (cited on 21 June 2006): http://adsabs.harvard.edu/abs/1935PASP . .47...15K. 5.1

[203] Kumar, S.S., "The Structure of Stars of Very Low Mass", Astrophys. J., 137, 1121, (1963). 1

[204] Kuranov, A.G., and Postnov, K.A., "Neutron stars in globular clusters: Formation and observational manifestations", Astron. Lett., 32, 393-405, (2006). Related online version (cited on 21 June 2006):

http://arXiv.org/abs/astro-ph/0605115. 3.4

[205] Kusenko, A., "Pulsar Kicks from Neutrino Oscillations", Int. J. Mod. Phys. D, 13, 20652084, (2004). 3.4

[206] Lai, D., "Neutron Star Kicks and Asymmetric Supernovae", in Blaschke, D., Glendenning, N.K., and Sedrakian, A., eds., Physics of Neutron Star Interiors, vol. 578 of Lecture Notes in Physics, 424, (Springer, Berlin, Germany; New York, U.S.A., 2001). Related online version (cited on 21 June 2006):

http://adsabs.harvard. edu/abs/2001LNP. . 578. .424L. 3.4

[207] Lai, D., Chernoff, D.F., and Cordes, J.M., "Pulsar Jets: Implications for Neutron Star Kicks and Initial Spins", Astrophys. J., 549, 1111-1118, (2001). 3.4

[208] Landau, L.D., and Lifshitz, E.M., Mechanics, vol. 1 of Course of Theoretical Physics, (Pergamon Press, Oxford, U.K.; New York, U.S.A., 1969), 2nd edition. 3.1 .1

[209] Landau, L.D., and Lifshitz, E.M., The Classical Theory of Fields, vol. 2 of Course of Theoretical Physics, (Pergamon Press, Oxford, U.K.; New York, U.S.A., 1975), 4th edition. 3.1.2, 3.1.3, 3.1.3

[210] Larson, S.L., Hiscock, W.A., and Hellings, R.W., "Sensitivity curves for spaceborne gravitational wave interferometers", Phys. Rev. D, 62, 062001, (2000). 11, 15

[211] Lattimer, J.M., and Prakash, M., "The Physics of Neutron Stars", Science, 304, 536-542, (2004). 1

[212] Lee, W.H., Ramirez-Ruiz, E., and Granot, J., "A Compact Binary Merger Model for the Short, Hard GRB 050509b", Astrophys. J. Lett., 630, L165-L168, (2005). Related online version (cited on 21 June 2006):

http://adsabs.harvard.edu/abs/2005ApJ . .630L.165L. 2.3

[213] Lee, W.H., Ramirez-Ruiz, E., and Page, D., "Dynamical Evolution of Neutrino-Cooled Accretion Disks: Detailed Microphysics, Lepton-Driven Convection, and Global Energetics", Astrophys. J., 632, 421-437, (2005). Related online version (cited on 21 June 2006): http: //adsabs.harvard.edu/abs/2005ApJ . . 632 . 421L. 2.3 
[214] Lentz, E.J., Baron, E., Hauschildt, P.H., and Branch, D., "Detectability of Hydrogen Mixing in Type Ia Supernova Premaximum Spectra", Astrophys. J., 580, 374-379, (2002). 7

[215] Lesaffre, P., Han, Z., Tout, C.A., Podsiadlowski, P., and Martin, R.G., "The C flash and the ignition conditions of Type Ia supernovae", Mon. Not. R. Astron. Soc., 368, 187-195, (2006). Related online version (cited on 21 June 2006): http://adsabs.harvard.edu/abs/2006MNRAS.368..187L. 7

[216] Li, W., Filippenko, A.V., Treffers, R.R., Riess, A.G., Hu, J., and Qiu, Y., "A High Intrinsic Peculiarity Rate among Type Ia Supernovae", Astrophys. J., 546, 734-743, (2001). Related online version (cited on 21 June 2006): http://adsabs.harvard.edu/abs/2001ApJ . .546. .734L. 7

[217] Li, X.-D., and van den Heuvel, P.J., "Evolution of white dwarf binaries: Supersoft X-ray sources and progenitors of type Ia upernovae", Astron. Astrophys., 322, L9-L12, (1997). 7

[218] Limongi, M., and Tornambè, A., "He stars and He-accreting CO white dwarfs", Astrophys. J., 371, 317-331, (1991). 7

[219] Lipunov, V.M., Astrophysics of Neutron Stars, (Springer, Berlin, Germany; New York, U.S.A., 1992). 5

[220] Lipunov, V.M., Bogomazov, A.I., and Abubekerov, M.K., "How abundant is the population of binary radio pulsars with black holes?", Mon. Not. R. Astron. Soc., 359, 1517-1523, (2005). 2, 13

[221] Lipunov, V.M., Nazin, S.N., Panchenko, I.E., Postnov, K.A., and Prokhorov, M.E., "The gravitational wave sky", Astron. Astrophys., 298, 677, (1995). Related online version (cited on 4 November 2006):

http://adsabs.harvard.edu/abs/1995A\&A...298. .677L. 6.2

[222] Lipunov, V.M., and Postnov, K.A., "Spectrum of gravitational radiation of binary systems", Sov. Astron., 31, 228-235, (1988). 10, 10

[223] Lipunov, V.M., Postnov, K.A., and Prokhorov, M.E., "The sources of gravitaional waves with continuous and discrete spectra", Astron. Astrophys., 176, L1-L4, (1987). 10

[224] Lipunov, V.M., Postnov, K.A., and Prokhorov, M.E., "The Scenario Machine: Binary Star Population Synthesis", Astrophys. Space Phys. Rev., 9, 1-178, (1996). Related online version (cited on 13 November 2006):

http://xray.sai.msu.ru/ mystery/articles/review/. 5.2

[225] Lipunov, V.M., Postnov, K.A., and Prokhorov, M.E., "The Scenario Machine: Restrictions on key parameters of binary evolution", Astron. Astrophys., 310, 489-507, (1996). 3.4, 3.4

[226] Lipunov, V.M., Postnov, K.A., and Prokhorov, M.E., "Black holes and gravitational waves: Possibilities for simultaneous detection using first-generation laser interferometers", Astron. Lett., 23, 492-497, (1997). 5.2, 6.1

[227] Lipunov, V.M., Postnov, K.A., and Prokhorov, M.E., "Formation and coalescence of relativistic binary stars: The effect of kick velocity", Mon. Not. R. Astron. Soc., 288, 245-259, (1997). 3.4, 4.1, 4.2, 4.2 
[228] Lipunov, V.M., Postnov, K.A., Prokhorov, M.E., and Osminkin, E.Y., "Binary Radiopulsars with Black Holes", Astrophys. J. Lett., 423, L121-L124, (1994). Related online version (cited on 08 December 2006):

http://adsabs .harvard. edu/abs/1994ApJ. .423L.121L. 2, 13

[229] Lipunov, V.M., Postnov, K.A., Prokhorov, M.E., Panchenko, I.E., and Jørgensen, H.E., "Evolution of the Double Neutron Star Merging Rate and the Cosmological Origin of GammaRay Burst Sources", Astrophys. J., 454, 593, (1995). Related online version (cited on 4 November 2006):

http://adsabs.harvard.edu/abs/1995ApJ . . 454. .593L. 6.2

[230] Livne, E., "Successive detonations in accreting white dwarfs as an alternative mechanism for type I supernovae", Astrophys. J., 354, L53-L55, (1990). Related online version (cited on 21 June 2006):

http: //adsabs.harvard.edu/abs/1990ApJ. . 354L. .53L. 7

[231] Livne, E., and Arnett, D., "Explosions of Sub-Chandrasekhar Mass White Dwarfs in Two Dimensions", Astrophys. J., 452, 62, (1995). Related online version (cited on 21 June 2006): http://adsabs.harvard.edu/abs/1995ApJ. . .452...62L. 7

[232] Livne, E., and Glasner, A., "Numerical simulations of off-center detonations in helium shells", Astrophys. J., 370, 272-281, (1991). 7

[233] Lombardi, J.C., Proulx, Z.F., Dooley, K.L., Theriault, E.M., Ivanova, N., and Rasio, F.A., "Stellar Collisions and Ultracompact X-Ray Binary Formation", Astrophys. J., 640, 441458, (2006). Related online version (cited on 21 June 2006):

http://adsabs.harvard.edu/abs/2006ApJ...640. .441L. 7

[234] Lommen, D., Yungelson, L.R., van den Heuvel, E., Nelemans, G., and Portegies Zwart, S., "Cygnus X-3 and the problem of the missing Wolf-Rayet X-ray binaries", Astron. Astrophys., 443, 231-241, (2005). Related online version (cited on 21 June 2006): http://adsabs.harvard.edu/abs/2005A\&A...443. .231L. 3.2 .3

[235] Lorén-Aguilar, P., Guerrero, J., Isern, J., Lobo, J.A., and García-Berro, E., "Gravitational wave radiation from the coalescence of white dwarfs", Mon. Not. R. Astron. Soc., 356, 627636, (2005). Related online version (cited on 21 June 2006):

http://adsabs.harvard.edu/abs/2005MNRAS.356. 627L. 7, 9

[236] Lorimer, D.R., "Binary and Millisecond Pulsars at the New Millennium", Living Rev. Relativity, 8, lrr-2005-7, (2005). URL (cited on 21 June 2006):

http://www. livingreviews.org/lrr-2005-7. 2.1

[237] Lorimer, D.R., Stairs, I.H., Freire, P.C., Cordes, J.M., Camilo, F., Faulkner, A.J., Lyne, A.G., Nice, D.J., Ransom, S.M., Arzoumanian, Z., Manchester, R.N., Champion, D.J., van Leeuwen, J., Mclaughlin, M.A., Ramachandran, R., Hessels, J.W., Vlemmings, W., Deshpande, A.A., Bhat, N.D., Chatterjee, S., Han, J.L., Gaensler, B.M., Kasian, L., Deneva, J.S., Reid, B., Lazio, T.J., Kaspi, V.M., Crawford, F., Lommen, A.N., Backer, D.C., Kramer, M., Stappers, B.W., Hobbs, G.B., Possenti, A., D'Amico, N., and Burgay, M., "Arecibo Pulsar Survey Using ALFA. II. The Young, Highly Relativistic Binary Pulsar J1906+0746", Astrophys. J., 640, 428-434, (2006). Related online version (cited on 21 June 2006): http://adsabs.harvard.edu/abs/2006ApJ...640..428L. 2.1

[238] Lubow, S.H., and Shu, F.H., "Gas dynamics of semidetached binaries", Astrophys. J., 198, $383,(1975) .11$ 
[239] Lyne, A.G., Burgay, M., Kramer, M., Possenti, A., Manchester, R.N., Camilo, F., McLaughlin, M.A., Lorimer, D.R., D’Amico, N., Joshi, B.C., Reynolds, J., and Freire, P.C.C., "A Double-Pulsar System: A Rare Laboratory for Relativistic Gravity and Plasma Physics", Science, 303, 1153-1157, (2004). 2.1, 2.1

[240] Lyne, A.G., Camilo, F., Manchester, R.N., Bell, J.F., Kaspi, V.M., D’Amico, N., McKay, N.P.F., Crawford, F., Morris, D.J., Sheppard, D.C., and Stairs, I.H., "The Parkes Multibeam Pulsar Survey: PSR J1811-1736, a pulsar in a highly eccentric binary system", Mon. Not. R. Astron. Soc., 312, 698-702, (2000). 2.1

[241] Lyne, A.G., and Lorimer, D.R., "High Birth Velocities of Radio Pulsars", Nature, 369, 127-129, (1994). 3.4

[242] MacDonald, J., "Are cataclysmic variables the progenitors of Type I supernovae?", Astrophys. J., 283, 241-248, (1984). Related online version (cited on 21 June 2006):

http://adsabs.harvard.edu/abs/1984ApJ . . 283. .241M. 12

[243] Madau, P., "Galaxy Evolution and the Cosmic Rate of Supernovae", in D'Odorico, S., Fontana, A., and Giallongo, E., eds., The Young Universe: Galaxy Formation and Evolution at Intermediate and High Redshift, Meeting at the Rome Astronomical Observatory, Monteporzio, Italy, 29 September - 3 October 1997, vol. 146 of ASP Conference Series, 289, (Astronomical Society of the Pacific, San Francisco, U.S.A., 1998). Related online version (cited on 21 June 2006):

http://adsabs.harvard.edu/abs/1998ASPC . 146 . .289M. 1

[244] Manchester, R.N., Lyne, A.G., Camilo, F., Bell, J.F., Kaspi, V.M., D’Amico, N., McKay, N.P.F., Crawford, F., Stairs, I.H., Possenti, A., Kramer, M., and Sheppard, D.C., "The Parkes multi-beam pulsar survey - I. Observing and data analysis systems, discovery and timing of 100 pulsars", Mon. Not. R. Astron. Soc., 328, 17-35, (2001). 1

[245] Mannucci, F., Della Valle, M., and Panagia, N., "Two populations of progenitors for Type Ia supernovae?", Mon. Not. R. Astron. Soc., 370, 773-783, (2006). Related online version (cited on 21 June 2006): http://adsabs.harvard.edu/abs/2005astro.ph.10315M. 7

[246] Marietta, E., Burrows, A., and Fryxell, B., "Type Ia Supernova Explosions in Binary Systems: The Impact on the Secondary Star and its Consequences", Astrophys. J. Suppl. Ser., 128, 615-650, (2000). Related online version (cited on 21 June 2006):

http://adsabs.harvard.edu/abs/2000ApJS. .128. .615M. 7

[247] Marsh, T.R., "Detached white-dwarf close-binary stars - CV's extended family", New Astron. Rev., 44, 119-124, (2000). Related online version (cited on 21 June 2006):

http://adsabs. harvard.edu/abs/2000NewAR. .44..119M. 8

[248] Marsh, T.R., Dhillon, V.S., and Duck, S.R., "Low-mass white dwarfs need friends: Five new double-degenerate close binary stars", Mon. Not. R. Astron. Soc., 275, 828, (1995). 8

[249] Marsh, T.R., Nelemans, G., and Steeghs, D., "Mass transfer between double white dwarfs", Mon. Not. R. Astron. Soc., 350, 113-128, (2004). Related online version (cited on 21 June 2006):

http://adsabs.harvard.edu/abs/2004MNRAS.350..113M. 7, 10, 11

[250] Marsh, T.R., Nelemans, G., and Steeghs, D., "Mass transfer between double white dwarfs", Mon. Not. R. Astron. Soc., 350, 113-128, (2004). Related online version (cited on 21 June 
2006):

http://adsabs.harvard.edu/abs/2004MNRAS.350..113M. 9

[251] Marsh, T.R., and Steeghs, D., "V407 Vul: A direct impact accretor", Mon. Not. R. Astron. Soc., 331, L7-L11, (2002). Related online version (cited on 21 June 2006):

http://adsabs.harvard.edu/abs/2002MNRAS.331L...7M. 11

[252] Masevich, A.G., Tutukov, A.V., and Yungelson, L.R., "Gravitational radiation and the evolution of dwarf binaries", Priroda, -, 68-76, (1981). Related online version (cited on 21 June 2006):

http://adsabs.harvard.edu/abs/1981Prir.......68M. In Russian. 10

[253] Massevitch, A.G., Tutukov, A.V., and Yungelson, L.R., "Evolution of massive close binaries and formation of neutron stars and black holes", Astrophys. Space Sci., 40, 115-133, (1976). 4.1

[254] McClintock, J.E., and Remillard, R.A., "Black Hole Binaries", in Lewin, W.H.G., and van der Klis, M., eds., Compact Stellar X-Ray Sources, vol. 39 of Cambridge Astrophysics Series, chapter 4, (Cambridge University Press, Cambridge, U.K., 2006). 4.2

[255] Miller, G.E., and Scalo, J.M., "The initial mass function and stellar birthrate in the solar neighborhood", Astrophys. J. Suppl. Ser., 41, 513-547, (1979). 1, 2.2, 5.1

[256] Mironovskii, V.N., "Gravitational Radiation of Double Stars", Sov. Astron., 9, 752, (1965). Related online version (cited on 21 June 2006):

http://adsabs.harvard.edu/abs/1965SvA....9.752M. 10

[257] Mitalas, R., "Effect of Asymmetric Explosion on Orbital Elements of Circular Binaries", Astron. Astrophys., 46, 323, (1976). Related online version (cited on 4 November 2006): http://adsabs.harvard.edu/abs/1976A\&A. . .46. .323M. 3.3

[258] Mochkovitch, R., Hernanz, M., Isern, J., and Martin, X., "Gamma-ray bursts as collimated jets from neutron star/black hole mergers", Nature, 361, 236-238, (1993). 2.3

[259] Mochkovitch, R., and Livio, M., "The coalescence of white dwarfs and type I supernovae. The merged configuration", Astron. Astrophys., 236, 378-384, (1990). Related online version (cited on 21 June 2006):

http://adsabs.harvard.edu/abs/1990A\&A...236..378M. 7, 7

[260] Morrison, R., and McCammon, D., "Interstellar photoelectric absorption cross sections, 0.0310 keV", Astrophys. J., 270, 119-122, (1983). Related online version (cited on 21 June 2006): http://adsabs.harvard.edu/abs/1983ApJ...270..119M. 11

[261] Motch, C., Haberl, F., Guillout, P., Pakull, M., Reinsch, K., and Krautter, J., "New cataclysmic variables from the ROSAT All-Sky Survey", Astron. Astrophys., 307, 459-469, (1996). Related online version (cited on 21 June 2006):

http://adsabs.harvard.edu/abs/1996A\&A...307. .459M. 11

[262] Muno, M.P., Baganoff, F.K., Bautz, M.W., Brandt, W.N., Broos, P.S., Feigelson, E.D., Garmire, G.P., Morris, M.R., Ricker, G.R., and Townsley, L.K., "A Deep Chandra Catalog of X-Ray Point Sources toward the Galactic Center", Astrophys. J., 589, 225-241, (2003). Related online version (cited on 21 June 2006):

http: //adsabs.harvard.edu/abs/2003ApJ...589..225M. 12 
[263] Muno, M.P., Clark, J.S., Crowther, P.A., Dougherty, S.M., de Grijs, R., Law, C., McMillan, S.L.W., Morris, M.R., Negueruela, I., Pooley, D., Portegies Zwart, S.F., and Yusef-Zadeh, F., "A Neutron Star with a Massive Progenitor in Westerlund 1", Astrophys. J. Lett., 636, L41-L44, (2006). Related online version (cited on 4 November 2006):

http://adsabs.harvard.edu/abs/2006ApJ . .636L . .41M. 4.2

[264] Nakar, E., Gal-Yam, A., and Fox, D.B., "The Local Rate and the Progenitor Lifetimes of Short-Hard Gamma-Ray Bursts: Synthesis and Predictions for the Laser Interferometer Gravitational-Wave Observatory", Astrophys. J., 650, 281-290, (2006). Related online version (cited on 4 November 2006):

http://adsabs.harvard.edu/abs/2006ApJ...650. .281N. 2.3

[265] Napiwotzki, R., Christlieb, N., Drechsel, H., Hagen, H.-J., Heber, U., Homeier, D., Karl, C., Koester, D., Leibundgut, B., Marsh, T.R., Moehler, S., Nelemans, G., Pauli, E.-M., Reimers, D., Renzini, A., and Yungelson, L.R., "Search for progenitors of supernovae type Ia with SPY", Astron. Nachr., 322, 411-418, (2001). 8.1

[266] Napiwotzki, R., Christlieb, N., Drechsel, H., Hagen, H.-J., Heber, U., Homeier, D., Karl, C., Koester, D., Leibundgut, B., Marsh, T.R., Moehler, S., Nelemans, G., Pauli, E.-M., Reimers, D., Renzini, A., and Yungelson, L.R., "SPY - The Eso Supernovae Type Ia Progenitor Survey", Messenger, 2003(112), 25-30, (2003). Related online version (cited on 21 June 2006):

http://adsabs.harvard.edu/abs/2003Msngr.112 ..25N. 8.1

[267] Napiwotzki, R., Karl, C.A., Nelemans, G., Yungelson, L.R., Christlieb, N., Drechsel, H., Heber, U., Homeier, D., Koester, D., Kruk, J., Leibundgut, B., Marsh, T.R., Moehler, S., Renzini, A., and Reimers, D., "New Results from the Supernova Ia Progenitor Survey", in Koester, D., and Moehler, S., eds., 14th European Workshop on White Dwarfs, Workshop in Kiel, Germany, 19 - 23 July 2004, vol. 334 of ASP Conference Series, 375, (Astronomical Society of the Pacific, San Francisco, U.S.A., 2005). Related online version (cited on 21 June 2006):

http://adsabs.harvard.edu/abs/2005ASPC. .334. .375N. 8.1

[268] Napiwotzki, R., Yungelson, L.R., Nelemans, G., Marsh, T.R., Leibundgut, B., Renzini, R., Homeier, D., Koester, D., Moehler, S., Christlieb, N., Reimers, D., Drechsel, H., Heber, U., Karl, C., and Pauli, E.-M., "Double Degenerates and Progenitors of Supernovae Type Ia", in Hilditch, R.W., Hensberge, H., and Pavlovski, K., eds., Spectroscopically and Spatially Resolving the Components of Close Binary Stars, Meeting in Dubrovnik, Croatia, 20 - 24 October 2003, vol. 318 of ASP Conference Series, 402-410, (Astronomical Society of the Pacific, San Francisco, U.S.A., 2004). Related online version (cited on 21 June 2006): http://adsabs.harvard.edu/abs/2004ASPC. .318. .402N. 8.1

[269] Narayan, R., "The birthrate and initial spin period of single radio pulsars", Astrophys. J., 319, 162-179, (1987). 2.2

[270] Narayan, R., Piran, T., and Shemi, A., "Neutron star and black hole binaries in the Galaxy", Astrophys. J. Lett., 379, L17-L20, (1991). Related online version (cited on 4 November 2006): http://adsabs.harvard.edu/abs/1991ApJ...379L..17N. 1, 2.2

[271] NASA Marshall Space Flight Center, "BATSE Web", project homepage, (2006). URL (cited on 21 June 2006):

http://www. batse.msfc.nasa.gov/batse/. 3 
[272] Nelemans, G., "AM CVn stars", in Hameury, J.-M., and Lasota, J.-P., eds., The Astrophysics of Cataclysmic Variables and Related Objects, Meeting in Strasbourg, France, 11 - 16 July 2004, vol. 330 of ASP Conference Series, 27, (Astronomical Society of the Pacific, San Francisco, U.S.A., 2005). Related online version (cited on 21 June 2006): http://adsabs . harvard. edu/abs/2005ASPC..330..27N. 8, 14

[273] Nelemans, G., "AM CVn stars", personal homepage, Radboud Universiteit Nijmegen, (2006). URL (cited on 21 June 2006):

http://www .astro.ru.nl/ nelemans/Research/AMCVn.html. 16

[274] Nelemans, G., "Gravitational waves", personal homepage, Radboud Universiteit Nijmegen, (2006). URL (cited on 21 June 2006):

http://www. astro.ru.nl/ nelemans/Research/GWR.html. 14

[275] Nelemans, G., "LISA Wiki Page", personal homepage, Radboud Universiteit Nijmegen, (2006). URL (cited on 21 June 2006):

http://www.astro.kun.nl/ nelemans/dokuwiki/doku.php. 8, 10

[276] Nelemans, G., "Preparing for the start of gravitational wave astrophysics", other, Kernfysisch Versneller Instituut, (2006). URL (cited on 21 June 2006):

http://www.kvi.nl/ berg/appsym4/Nelemans.pdf. 8

[277] Nelemans, G., and Jonker, P.G., "Ultra-compact (X-ray) binaries", New Astron. Rev., submitted, (2006). Related online version (cited on 21 June 2006):

http://adsabs.harvard.edu/abs/2006astro.ph..5722N. 7,8

[278] Nelemans, G., Jonker, P.G., Marsh, T.R., and van der Klis, M., "Optical spectra of the carbon-oxygen accretion discs in the ultra-compact X-ray binaries 4U 0614+09, 4U 1543624 and 2S 0918-549", Mon. Not. R. Astron. Soc., 348, L7-L11, (2004). Related online version (cited on 21 June 2006):

http://adsabs.harvard.edu/abs/2004MNRAS.348L . .7N. 7

[279] Nelemans, G., Jonker, P.G., and Steeghs, D., "Optical spectroscopy of (candidate) ultracompact X-ray binaries: Constraints on the composition of the donor stars", Mon. Not. R. Astron. Soc., 370, 255-262, (2006). Related online version (cited on 21 June 2006): http://arXiv.org/abs/astro-ph/0604597. 7

[280] Nelemans, G., Portegies Zwart, S.F., Verbunt, F., and Yungelson, L.R., "Population synthesis for double white dwarfs. II. Semi-detached systems: AM CVn stars", Astron. Astrophys., 368, 939-949, (2001). Related online version (cited on 21 June 2006):

http://adsabs.harvard.edu/abs/2001A\&A...368..939N. 7, 10

[281] Nelemans, G., Steeghs, D., and Groot, P.J., "Spectroscopic evidence for the binary nature of AM CVn", Mon. Not. R. Astron. Soc., 326, 621-627, (2001). Related online version (cited on 21 June 2006):

http://adsabs.harvard.edu/abs/2001MNRAS.326. .621N. 10

[282] Nelemans, G., Tauris, T.M., and van den Heuvel, E.P.J., "Constraints on mass ejection in black hole formation derived from black hole X-ray binaries", Astron. Astrophys., 352, L87-L90, (1999). 3.4

[283] Nelemans, G., and Tout, C.A., "Reconstructing the evolution of white dwarf binaries: Further evidence for an alternative algorithm for the outcome of the common-envelope phase in close binaries", Mon. Not. R. Astron. Soc., 356, 753-764, (2005). Related online version (cited on 
21 June 2006):

http://adsabs.harvard.edu/abs/2005MNRAS.356 . 753N. 3.5, 14

[284] Nelemans, G., Verbunt, F., Yungelson, L.R., and Portegies Zwart, S.F., "Reconstructing the evolution of double helium white dwarfs: Envelope loss without spiral-in", Astron. Astrophys., 360, 1011-1018, (2000). Related online version (cited on 13 November 2006): http://adsabs.harvard.edu/abs/2000A\&A..360.1011N. 3.5, 14

[285] Nelemans, G., Yungelson, L.R., and Portegies Zwart, S.F., "The gravitational wave signal from the Galactic disk population of binaries containing two compact objects", Astron. Astrophys., 375, 890-898, (2001). Related online version (cited on 21 June 2006):

http://adsabs.harvard.edu/abs/2001A\&A..375. 890N. 3.4, 5.2, 5.2, 7, 7, 8, 9, 10,6, $10,10,11,12,13,10,14,11,11$

[286] Nelemans, G., Yungelson, L.R., and Portegies Zwart, S.F., "Short-Period AM CVn systems as optical, X-ray and gravitational-wave sources", Mon. Not. R. Astron. Soc., 349, 181-192, (2004). Related online version (cited on 21 June 2006):

http://adsabs.harvard.edu/abs/2004MNRAS.349.181N. 9, 10, 10, 10, 10, 14, 15, 11, 16, $12,17,12$

[287] Nelemans, G., Yungelson, L.R., Portegies Zwart, S.F., and Verbunt, F., "Population synthesis for double white dwarfs. I. Detached systems", Astron. Astrophys., 365, 491-507, (2001). 7, 8.1

[288] Nelson, L.A., Rappaport, S.A., and Joss, P.C., "The evolution of ultrashort period binary systems", Astrophys. J., 304, 231-240, (1986). Related online version (cited on 21 June 2006):

http://adsabs.harvard.edu/abs/1986ApJ...304..231N. 7

[289] Nice, D.J., Sayer, R.W., and Taylor, J.H., "PSR J1518+4904: A Mildly Relativistic Binary Pulsar System", Astrophys. J. Lett., 466, L87-L90, (1996). 2.1

[290] Nice, D.J., Splaver, E.M., and Stairs, I.H., "Arecibo Measurements of Pulsar-White Dwarf Binaries: Evidence for Heavy Neutron Stars", in Rasio, F.A., and Stairs, I.H., eds., Binary Radio Pulsars, Meeting at the Aspen Center for Physics, Colorado, 12 - 16 January 2004, vol. 328 of ASP Conference Series, 371, (Astronomical Society of the Pacific, San Francisco, U.S.A., 2004). Related online version (cited on 21 June 2006):

http://adsabs.harvard.edu/abs/2005ASPC. .328..371N. 2.1

[291] Nomoto, K., and Iben Jr, I., "Carbon ignition in a rapidly accreting degenerate dwarf: A clue to the nature of the merging process in close binaries", Astrophys. J., 297, 531-537, (1985). Related online version (cited on 21 June 2006):

http://adsabs.harvard.edu/abs/1985ApJ...297..531N. 7

[292] Nomoto, K., and Kondo, Y., "Conditions for accretion-induced collapse of white dwarfs", Astrophys. J. Lett., 367, L19-L22, (1991). Related online version (cited on 21 June 2006): http://adsabs. harvard.edu/abs/1991ApJ . . .367L..19N. 1

[293] Nugis, T., and Lamers, H.J.G.L.M., "Mass-loss rates of Wolf-Rayet stars as a function of stellar parameters", Astron. Astrophys., 360, 227-244, (2000). 2

[294] Nussbaumer, H., and Orr, A., eds., Star Clusters: Saas-Fee Advanced Course 22, Lecture Notes of the Saas-Fee Advanced Course 22, Les Diablerets, Switzerland, April 6-11, 1992, Saas-Fee Advanced Courses, (Springer, Berlin, Germany; New York, U.S.A., 1994). 3.2 
[295] Oechslin, R., and Janka, T., "Short Gamma-Ray Bursts from Binary Neutron Star Mergers", (April, 2006). URL (cited on 21 June 2006):

http://arXiv.org/abs/astro-ph/0604562. 2.3

[296] Orosz, J.A, "Inventory of black hole binaries", in van der Hucht, K.A., Herrero, A., and Esteban, C., eds., A Massive Star Odyssey: From Main Sequence to Supernova, Conference in Lanzarote, Spain, 24-28 June 2002, vol. 212 of IAU Symposia, 365, (Astronomical Society of the Pacific, San Francisco, U.S.A., 2003). Related online version (cited on 4 November 2006):

http://arXiv.org/abs/astro-ph/0209041. 4.2

[297] O’Shaughnessy, R., Kim, C., Fragos, T., Kalogera, V., and Belczynski, K., "Constraining Population Synthesis Models via the Binary Neutron Star Population", Astrophys. J., 633, 1076-1084, (2005). Related online version (cited on 21 June 2006): http: //adsabs . harvard. edu/abs/2005ApJ . .633.10760. 5.2, 1

[298] Ostriker, J.P., "Common Envelope Binaries", Structure and Evolution of Close Binary Systems (IAU Symposium 73), Cambridge, England, 28 July - 1 August, 1975, conference paper, (1976). 3.5

[299] Paciesas, W.S., Meegan, C.A., Pendleton, G.N.and Briggs, M.S., Kouveliotou, C., Koshut, T.M., Lestrade, J.P., McCollough, M.L., Brainerd, J.J., Hakkila, J., Henze, W., Preece, R.D., Connaughton, V., Kippen, R.M., Mallozzi, R.S., Fishman, G.J., Richardson, G.A., and Sahi, M., "The Fourth BATSE Gamma-Ray Burst Catalog (Revised)", Astrophys. J. Suppl. Ser., 122, 465-495, (1999). 2.3

[300] Paczyński, B., "Gravitational Waves and the Evolution of Close Binaries", Acta Astron., 17, 287, (1967). Related online version (cited on 21 June 2006):

http://adsabs.harvard.edu/abs/1967AcA...17 .287P. 7, 8, 9

[301] Paczyński, B., "Common envelope binaries", in Eggleton, P.P., Mitton, S., and Whelan, J., eds., Structure and Evolution of Close Binary Systems (IAU Symposium 73), Conference in Cambridge, U.K., 28 July - 1 August 1975, 75-80, (D. Reidel, Dordrecht, Netherlands; Boston, U.S.A., 1976). 3.5, 7, 8

[302] Paczyński, B., "Gamma-ray bursters at cosmological distances", Astrophys. J. Lett., 308, L43-L46, (1986). 2.3

[303] Paczyński, B., "Cosmological gamma-ray bursts", Acta Astron., 41, 257-267, (1991). 2.3

[304] Panagia, N., Van Dyk, S.D., Weiler, K.W., Sramek, R.A., Stockdale, C.J., and Murata, K.P., "A Search for Radio Emission from Type Ia Supernovae", Astrophys. J., submitted, (2006). Related online version (cited on 21 June 2006): http://arXiv.org/abs/astro-ph/0603808. 7

[305] Panchenko, I.E., Lipunov, V.M., Postnov, K.A., and Prokhorov, M.E., "Stellar evolution, GRB and their hosts", Astron. Astrophys. Suppl., 138, 517-518, (1999). 2.3

[306] Pérez-González, P.G., Zamorano, J., Gallego, J., Aragón-Salamanca, A., and Gil de Paz, A., "Spatial Analysis of the $\mathrm{H} \alpha$ Emission in the Local Star-Forming UCM Galaxies", Astrophys. J., 591, 827-842, (2003). 2.2

[307] Perlmutter, S., Aldering, G., Goldhaber, G., Knop, R.A., Nugent, P.E., Castro, P.G., Deustua, S., Fabbro, S., Goobar, A., Groom, D.E., Hook, I.M., Kim, A.G., Kim, M.Y., 
Lee, J.C., Nunes, N.J., Pain, R., Pennypacker, C.R., Quimby, R., Lidman, C., Ellis, R.S., Irwin, M., McMahon, R.G., Ruiz-Lapuente, P., Walton, N., Schaefer, B., Boyle, B.J., Filippenko, A.V., Matheson, T., Fruchter, A.S., Panagia, N., Newberg, H.J.M., Couch, W.J., and Project, The Supernova Cosmology, "Measurements of Omega and Lambda from 42 HighRedshift Supernovae", Astrophys. J., 517, 565-586, (1999). Related online version (cited on 21 June 2006):

http://adsabs.harvard.edu/abs/1999ApJ...517..565P. 1

[308] Peters, P.C., "Gravitational Radiation and the Motion of Two Point Masses", Phys. Rev., 136, B1224-B1232, (1964). 2.1, 3.1.4, 3.1.4

[309] Pfahl, E., Podsiadlowski, P., and Rappaport, S., "Relativistic Binary Pulsars with Black Hole Companions", Astrophys. J., 628, 343-352, (2005). Related online version (cited on 21 June 2006):

http://adsabs.harvard.edu/abs/2005ApJ. . .628. .343P. 2, 13

[310] Pfahl, E., Rappaport, S., and Podsiadlowski, P., "A Comprehensive Study of Neutron Star Retention in Globular Clusters", Astrophys. J., 573, 283-305, (2002). Related online version (cited on 4 November 2006):

http://adsabs.harvard.edu/abs/2002ApJ...573. . 283P. 3.4

[311] Pfahl, E., Rappaport, S., and Podsiadlowski, P., "The Galactic Population of Low- and Intermediate-Mass X-Ray Binaries", Astrophys. J., 597, 1036-1048, (2003). Related online version (cited on 21 June 2006):

http://adsabs.harvard.edu/abs/2003ApJ...597.1036P. 7

[312] Pfahl, E., Rappaport, S., Podsiadlowski, P., and Spruit, H., "A New Class of High-Mass X-Ray Binaries: Implications for Core Collapse and Neutron Star Recoil", Astrophys. J., 574, 364-376, (2002). Related online version (cited on 4 November 2006):

http://adsabs.harvard.edu/abs/2002ApJ. . .574 . .364P. 3.4

[313] Phinney, E.S., "The rate of neutron star binary mergers in the universe: Minimal predictions for gravity wave detectors", Astrophys. J. Lett., 380, L17-L21, (1991). 1, 2.2

[314] Piersanti, L., Cassisi, S., Iben Jr, I., and Tornambé, A., "On the Very Long Term Evolutionary Behavior of Hydrogen-accreting Low-Mass CO White Dwarfs", Astrophys. J. Lett., 521, L59-L62, (1999). 7

[315] Piersanti, L., Gagliardi, S., Iben Jr, I., and Tornambé, A., "Carbon-Oxygen White Dwarf Accreting CO-Rich Matter. II. Self-Regulating Accretion Process up to the Explosive Stage", Astrophys. J., 598, 1229-1238, (2003). Related online version (cited on 21 June 2006): http://adsabs .harvard.edu/abs/2003ApJ . . 598.1229P. 7

[316] Piersanti, L., Gagliardi, S., Iben Jr, I., and Tornambé, A., "Carbon-Oxygen White Dwarfs Accreting CO-Rich Matter. I. A Comparison between Rotating and Nonrotating Models", Astrophys. J., 583, 885-901, (2003). Related online version (cited on 21 June 2006): http://adsabs.harvard.edu/abs/2003ApJ...583. .885P. 7

[317] Piran, T., and Guetta, D., "The rate and luminosity function of Short GRBs", in Holt, S.S., Gehrels, N., and Nousek, J.A., eds., Gamma-Ray Bursts in the Swift Era, Sixteenth Maryland Astrophysics Conference, Washington, DC, 29 November - 2 December 2005, vol. 836 of AIP Conference Proceedings, (American Institute of Physics, Melville, U.S.A., 2006). Related online version (cited on 21 June 2006):

http://adsabs.harvard.edu/abs/2006astro.ph. .2208P. 2.3 
[318] Podsiadlowski, P., Han, Z., and Rappaport, S.A., "Cataclysmic variables with evolved secondaries and the progenitors of AM CVn stars", Mon. Not. R. Astron. Soc., 340, 1214-1228, (2003). Related online version (cited on 4 November 2006): http://adsabs.harvard.edu/abs/2003MNRAS.340.1214P. 9

[319] Podsiadlowski, P., Langer, N., Poelarends, A.J.T., Rappaport, S., Heger, A., and Pfahl, E., "The Effects of Binary Evolution on the Dynamics of Core Collapse and Neutron Star Kicks", Astrophys. J., 612, 1044-1051, (2004). 3.4

[320] Podsiadlowski, P., Rappaport, S., and Han, Z., "On the formation and evolution of black hole binaries", Mon. Not. R. Astron. Soc., 341, 385-404, (2003). 3.5, 7

[321] Podsiadlowski, P., Rappaport, S., and Pfahl, E.D., "Evolutionary Sequences for Low- and Intermediate-Mass X-Ray Binaries", Astrophys. J., 565, 1107-1133, (2002). Related online version (cited on 21 June 2006):

http://adsabs.harvard.edu/abs/2002ApJ...565.1107P. 7

[322] Podsiadlowski, Ph., Dewi, J.D.M., Lesaffre, P., Miller, J.C., Newton, W.G., and Stone, J.R., "The Double Pulsar J0737-3039: Testing the Neutron Star Equation of State", Mon. Not. R. Astron. Soc., 361, 1243-1249, (2005). Related online version (cited on 21 June 2006): http://arXiv.org/abs/astro-ph/0506566. 3.4

[323] Politano, M., and Weiler, K.P., "The Distribution of Secondary Masses in Post-CommonEnvelope Binaries: A Potential Test of Disrupted Magnetic Braking", Astrophys. J. Lett., 641, L137-L140, (2006). Related online version (cited on 21 June 2006):

http://adsabs .harvard.edu/abs/2006ApJ . .641L.137P. 3.1.5

[324] Pols, O.R., Schröder, K.-P., Hurley, J.R., Tout, C.A., and Eggleton, P.P., "Stellar evolution models for $Z=0.0001$ to 0.03", Mon. Not. R. Astron. Soc., 298, 525-536, (1998). Related online version (cited on 21 June 2006):

http://adsabs.harvard.edu/abs/1998MNRAS.298. .525P. 1

[325] Popov, S.B., and Prokhorov, M.E., "Population synthesis in astrophysics", (November, 2004). URL (cited on 21 June 2006):

http://arXiv.org/abs/astro-ph/0411792. 5.2

[326] Popova, E.I., Tutukov, A.V., and Yungelson, L.R., "Study of physical properties of spectroscopic binary stars", Astrophys. J. Suppl. Ser., 88, 55-80, (1982). 5.1, 5.1

[327] Portegies Zwart, S.F., Verbunt, F., and Ergma, E., "The formation of black-holes in low-mass X-ray binaries", Astron. Astrophys., 321, 207-212, (1997). 4.2

[328] Portegies Zwart, S.F., and Yungelson, L.R., "Formation and Evolution of Binary Neutron Stars", Astron. Astrophys., 332, 173-188, (1998). 3.4, 6, 4.1, 5.2, 5.2

[329] Postnov, K.A., and Prokhorov, M.E., "Galactic binary gravitational wave noise within LISA frequency band", Astrophys. J., 494, 674-679, (1998). Related online version (cited on 21 June 2006): http://arXiv.org/abs/astro-ph/9801034. 10

[330] Postnov, K.A., and Prokhorov, M.E., "Binary black hole formaiton and merging", in Trân Than Vân, J., Dumarchez, J., Raynoud, S., Salomon, C., Thorsett, S., and Vinet, J.Y., eds., Gravitational Waves and Experimental Gravity, XXXIVth Rencontres de Moriond, Les Arcs, France, 23 - 30 January 1999, 113-118, (World Publishers, Hanoi, Vietnam, 2000). 3.4, 4.2 
[331] Prialnik, D., and Kovetz, A., "An extended grid of multicycle nova evolution models", Astrophys. J., 445, 789-810, (1995). Related online version (cited on 21 June 2006):

http://adsabs.harvard.edu/abs/1995ApJ . . 445. .789P. 7

[332] Prince, T.A., Anderson, S.B., Kulkarni, S.R., and Wolszczan, A., "Timing observations of the 8 hour binary pulsar $2127+11 \mathrm{C}$ in the globular cluster M15", Astrophys. J. Lett., 374, L41-L44, (1991). 2.1

[333] Pringle, J.E., "Soft X-ray emission from dwarf novae", Mon. Not. R. Astron. Soc., 178, 195-202, (1977). Related online version (cited on 21 June 2006): http://adsabs .harvard.edu/abs/1977MNRAS .178. .195P. 11

[334] Prokhorov, M.E., and Postnov, K.A., "Direct observation of the kick during the birth of a neutron star in the binary pulsar PSR B1259-63", Astron. Lett., 23, 439-444, (1997). 3.4

[335] Raguzova, N.V., and Popov, S.B., "Be X-ray binaries and candidates", Astron. Astrophys. Trans., 24, 151-185, (2005). Related online version (cited on 21 June 2006):

http://adsabs.harvard.edu/abs/2005A\&AT...24..151R. 5

[336] Ramsay, G., Brocksopp, C., Groot, P.J., Hakala, P., Lehto, H.J., Marsh, T.R., Napiwotzki, R., Nelemans, G., Potter, S., Slee, B., Steeghs, D., and Wu, K., "Recent observational progress in AM CVn binaries", (October, 2006). URL (cited on 4 November 2006): http://arXiv.org/abs/astro-ph/0610357. 12.2

[337] Ramsay, G., Groot, P.J., Marsh, T.R., Nelemans, G., Steeghs, D., and Hakala, P., "XMMNewton observations of AM CVn binaries: V396 Hya and SDSS J1240-01", Astron. Astrophys., 457, 623-627, (2006). Related online version (cited on 4 November 2006): http://adsabs.harvard.edu/abs/2006A\&A...457..623R. 9

[338] Ramsay, G., and Hakala, P., "RApid Temporal Survey (RATS) - I. Overview and first results", Mon. Not. R. Astron. Soc., 360, 314-321, (2005). Related online version (cited on 21 June 2006):

http://adsabs .harvard.edu/abs/2005MNRAS.360..314R. 12.2

[339] Rappaport, S., Di Stefano, R., and Smith, J.D., "Formation and evolution of luminous supersoft X-ray sources", Astrophys. J., 426, 692-703, (1994). Related online version (cited on 21 June 2006):

http://adsabs.harvard.edu/abs/1994ApJ . . 426. .692R. 7

[340] Rappaport, S., and Joss, P.C., "The lower main sequence and the nature of secondary stars in ultracompact binaries", Astrophys. J., 283, 232-240, (1984). Related online version (cited on 21 June 2006):

http://adsabs.harvard.edu/abs/1984ApJ ..283..232R. 8, 9

[341] Rasio, F.A., and Livio, M., "On the Formation and Evolution of Common Envelope Systems", Astrophys. J., 471, 366, (1996). 3.5

[342] Rasio, F.A., Pfahl, E.D., and Rappaport, S., "Formation of Short-Period Binary Pulsars in Globular Clusters", Astrophys. J. Lett., 532, L47-L50, (2000). Related online version (cited on 21 June 2006):

http://adsabs.harvard.edu/abs/2000ApJ . . 532L. .47R. 7

[343] Rees, M.J., "Opacity-limited hierarchical fragmentation and the masses of protostars", Mon. Not. R. Astron. Soc., 176, 483-486, (1976). 1 
[344] Refsdal, S., Roth, M.L., and Weigert, A., "On the binary system AS Eri", Astron. Astrophys., 36, 113-122, (1974). Related online version (cited on 21 June 2006):

http://adsabs.harvard.edu/abs/1974A\&A....36..113R. 3.5

[345] Remillard, R.A., and McClintock, J.E., "X-Ray Properties of Black-Hole Binaries", Annu. Rev. Astron. Astrophys., 44, (2006). Related online version (cited on 21 June 2006):

http://adsabs.harvard.edu/abs/2006astro.ph..6352R. 4.2

[346] Ricci, F., and Brillet, A., "A Review of Gravitational Wave Detectors", Annu. Rev. Nucl. Part. Sci., 47, 111-156, (1997). Related online version (cited on 21 June 2006):

http://adsabs . harvard.edu/abs/1997ARNPS . .47 . .111R. 1

[347] Riess, A.G., Filippenko, A.V., Challis, P., Clocchiatti, A., Diercks, A., Garnavich, P.M., Gilliland, R.L., Hogan, C.J., Jha, S., Kirshner, R.P., Leibundgut, B., Phillips, M.M., Reiss, D., Schmidt, B.P., Schommer, R.A., Smith, R.C., Spyromilio, J., Stubbs, C., Suntzeff, N.B., and Tonry, J., "Observational Evidence from Supernovae for an Accelerating Universe and a Cosmological Constant", Astron. J., 116, 1009-1038, (1998). Related online version (cited on 21 June 2006):

http://adsabs.harvard.edu/abs/1998AJ....116.1009R. 1

[348] Ritossa, C., García-Berro, E., and Iben Jr, I., "On the Evolution of Stars That Form ElectronDegenerate Cores Processed by Carbon Burning. II. Isotope Abundances and Thermal Pulses in a $10 M_{\odot}$ Model with an ONe Core and Applications to Long-Period Variables, Classical Novae, and Accretion-Induced Collapse", Astrophys. J., 460, 489, (1996). Related online version (cited on 21 June 2006):

http://adsabs.harvard.edu/abs/1996ApJ . .460..489R. 1

[349] Ritossa, C., García-Berro, E., and Iben Jr, I., "On the Evolution of Stars that Form Electrondegenerate Cores Processed by Carbon Burning. V. Shell Convection Sustained by Helium Burning, Transient Neon Burning, Dredge-Out, URCA Cooling, and Other Properties of an $11 M_{\odot}$ Population I Model Star", Astrophys. J., 515, 381-397, (1999). Related online version (cited on 21 June 2006):

http: //adsabs . harvard. edu/abs/1999ApJ...515. .381R. 1, 3.4

[350] Ritter, H., and Kolb, U., "Catalogue of cataclysmic binaries, low-mass X-ray binaries and related objects (Seventh edition)", Astron. Astrophys., 404, 301-303, (2003). Related online version (cited on 21 June 2006):

http://adsabs.harvard.edu/abs/2003A\&A...404_.301R. 8

[351] Ritter, H., and Kolb, U., "Catalogue of Cataclysmic Binaries, Low-Mass X-Ray Binaries and Related Objects", project homepage, Open University, (2006). URL (cited on 21 June 2006): http://physics.open.ac.uk/RKcat/. 8

[352] Robinson, E.L., and Shafter, A.W., "An Upper Limit to the Space Density of Short-period Noninteracting Binary White Dwarfs", Astrophys. J., 322, 296, (1987). Related online version (cited on 21 June 2006):

http://adsabs.harvard.edu/abs/1987ApJ...322..296R. 8

[353] Roelofs, G.H.A., Groot, P.J., Marsh, T.R., Steeghs, D., Barros, S.C.C., and Nelemans, G., "SDSS J124058.03-015919.2: A new AM CVn star with a 37-min orbital period", Mon. Not. R. Astron. Soc., 361, 487-494, (2005). Related online version (cited on 21 June 2006): http://adsabs .harvard. edu/abs/2005MNRAS.361..487R. 12.2 
[354] Roelofs, G.H.A., Groot, P.J., Nelemans, G., Marsh, T.R., and Steeghs, D., "Kinematics of the ultra-compact helium accretor AM Canum Venaticorum", (June, 2006). URL (cited on 4 November 2006):

http://arXiv.org/abs/astro-ph/0606327. 9

[355] Rogan, A., and Bose, S., "Parameter estimation of binary compact objects with LISA: Effects of time-delay interferometry, Doppler modulation, and frequency evolution", (May, 2006). URL (cited on 21 June 2006):

http://arXiv.org/abs/astro-ph/0605034. 12.2

[356] Romani, R.W., "A unified model of neutron-star magnetic fields", Nature, 347, 741-743, (1990). 5

[357] Romani, R.W., "Neutron Star Magnetic Fields", in Fruchter, A.S., Tavani, M., and Backer, D.C., eds., Millisecond Pulsars: A Decade of Surprise, Conference in Aspen, Colorado, 3 7 January 1994, vol. 72 of ASP Conference Series, 288, (Astronomical Society of the Pacific, San Francisco, U.S.A., 1995). 5

[358] Ruderman, M.A., and Shaham, J., "Fate of very low-mass secondaries in accreting binaries and the 1.5-ms pulsar", Nature, 304, 425-427, (1983). 7

[359] Ruiz-Lapuente, P., and Canal, R., "Type Ia Supernova Counts at High $z$ : Signatures of Cosmological Models and Progenitors", Astrophys. J. Lett., 497, L57-L60, (1998). Related online version (cited on 21 June 2006):

http://adsabs. harvard.edu/abs/1998ApJ . . 497L. . 57R. 1

[360] Ruiz-Lapuente, P., Comeron, F., Méndez, J., Canal, R., Smartt, S.J., Filippenko, A.V., Kurucz, R.L., Chornock, R., Foley, R.J., Stanishev, V., and Ibata, R., "The binary progenitor of Tycho Brahe's 1572 supernova", Nature, 431, 1069-1072, (2004). 14

[361] Sackett, P.D., "Does the Milky Way Have a Maximal Disk?", Astrophys. J., 483, 103, (1997). Related online version (cited on 21 June 2006):

http://adsabs.harvard.edu/abs/1997ApJ ...483..103S. 10

[362] Saffer, R.A., Liebert, J., and Olszewski, E.W., "Discovery of a close detached binary DA white dwarf system", Astrophys. J., 334, 947-957, (1988). 8

[363] Saffer, R.A., Livio, M., and Yungelson, L.R., "Close Binary White Dwarf Systems: Numerous New Detections and Their Interpretation", Astrophys. J., 502, 394, (1998). 8

[364] Saio, H., and Nomoto, K., "Off-Center Carbon Ignition in Rapidly Rotating, Accreting Carbon-Oxygen White Dwarfs", Astrophys. J., 615, 444-449, (2004). Related online version (cited on 21 June 2006):

http://adsabs.harvard.edu/abs/2004ApJ...615..444S. 7

[365] Salpeter, E.E., "The Luminosity Function and Stellar Evolution", Astrophys. J., 121, 161167, (1955). Related online version (cited on 21 June 2006): http://adsabs.harvard.edu/abs/1955ApJ...121..161S. 1

[366] Sandage, A., "The redshift-distance relation. II. The Hubble diagram and its scatter for firstranked cluster galaxies: A formal value for $q_{0} "$, Astrophys. J., 178, 1-24, (1972). Related online version (cited on 21 June 2006):

http://adsabs.harvard.edu/abs/1972ApJ...178....1S. 11 
[367] Sandquist, E.L., Taam, R.E., and Burkert, A., "On the Formation of Helium Double Degenerate Stars and Pre-Cataclysmic Variables", Astrophys. J., 533, 984-997, (2000). Related online version (cited on 21 June 2006):

http://adsabs.harvard. edu/abs/2000ApJ...533. .984S. 3.5, 7

[368] Sandquist, E.L., Taam, R.E., Chen, X., Bodenheimer, P., and Burkert, A., "Double Core Evolution. X. Through the Envelope Ejection Phase", Astrophys. J., 500, 909, (1998). Related online version (cited on 21 June 2006):

http://adsabs.harvard.edu/abs/1998ApJ...500..909S. 7

[369] Savonije, G.J., de Kool, M., and van den Heuvel, E.P.J., "The minimum orbital period for ultra-compact binaries with helium burning secondaries", Astron. Astrophys., 155, 51-57, (1986). Related online version (cited on 21 June 2006):

http://adsabs.harvard.edu/abs/1986A\&A...155...51S. 7, 7

[370] Schatzman, E., "A theory of the role of magnetic activity during star formation", Ann. Astrophys., 25, 18, (1962). Related online version (cited on 21 June 2006):

http://adsabs . harvard.edu/abs/1962AnAp...25...18S. 3.1 .5

[371] Schiminovich, D., Ilbert, O., Arnouts, S., Milliard, B., Tresse, L., Le Fèvre, O., Treyer, M., Wyder, T.K., Budavári, T., Zucca, E., Zamorani, G., Martin, D.C., Adami, C., Arnaboldi, M., Bardelli, S., Barlow, T., Bianchi, L., Bolzonella, M., Bottini, D., Byun, Y.-I., Cappi, A., Contini, T., Charlot, S., Donas, J., Forster, K., Foucaud, S., Franzetti, P., Friedman, P.G., Garilli, B., Gavignaud, I., Guzzo, L., Heckman, T.M., Hoopes, C., Iovino, A., Jelinsky, P., Le Brun, V.and Lee, Y.-W., Maccagni, D., Madore, B.F., Malina, R.and Marano, B., Marinoni, C., McCracken, H.J., Mazure, A., Meneux, B., Morrissey, P., Neff, S., Paltani, S.and Pellò, R., Picat, J.P., Pollo, A., Pozzetti, L., Radovich, M., Rich, R.M., Scaramella, R., Scodeggio, M., Seibert, M., Siegmund, O., Small, T., Szalay, A.S., Vettolani, G., Welsh, B., Xu, C.K., and Zanichelli, A., "The GALEX-VVDS Measurement of the Evolution of the Far-Ultraviolet Luminosity Density and the Cosmic Star Formation Rate", Astrophys. J. Lett., 619, L47-L50, (2005). 2.2

[372] Schröder, K.-P., Pauli, E.-M., and Napiwotzki, R., "A population model of the solar neighbourhood thin disc white dwarfs", Mon. Not. R. Astron. Soc., 354, 727-736, (2004). Related online version (cited on 21 June 2006):

http://adsabs.harvard.edu/abs/2004MNRAS.354 . .727S. 8.1

[373] Schutz, B.F., "Observational evidence for supermassive black hole binaries", in Centrella, J.M., ed., The Astrophysics of Gravitational Wave Sources, College Park, Maryland, $24-26$ April 2003, vol. 686 of AIP Conference Proceedings, 3-29, (American Institute of Physics, Melville, U.S.A., 2003). 1

[374] Seto, N., "Long-term operation of the Laser Interferometer Space Antenna and Galactic close white dwarf binaries", Mon. Not. R. Astron. Soc., 333, 469-474, (2002). Related online version (cited on 21 June 2006):

http: //adsabs .harvard.edu/abs/2002MNRAS.333 . 469S. 10, 10

[375] Shapiro, S.L., and Teukolsky, S.A., Black Holes, White Dwarfs, and Neutron Stars, (Wiley, New York, U.S.A., 1983). 1

[376] Shklovskii, I.S., "Possible causes of the secular increase in pulsar periods", Sov. Astron., 13, $562-565,(1970)$. 3.4 
[377] Siess, L., "Evolution of massive AGB stars. I. Carbon burning phase", Astron. Astrophys., 448, 717-729, (2006). Related online version (cited on 21 June 2006):

http://adsabs.harvard.edu/abs/2006A\&A...448..717S. 1

[378] Skumanich, A., "Time Scales for Ca II Emission Decay, Rotational Braking, and Lithium Depletion", Astrophys. J., 171, 565-567, (1972). Related online version (cited on 21 June 2006):

http://adsabs.harvard.edu/abs/1972ApJ...171. .565S. 3.1.5

[379] Smak, J., "Light variability of HZ 29", Acta Astron., 17, 255-270, (1967). Related online version (cited on 21 June 2006):

http://adsabs.harvard.edu/abs/1967AcA....17..255S. 7

[380] Soberman, G.E., Phinney, E.S., and van den Heuvel, E.P.J., "Stability criteria for mass transfer in binary stellar evolution", Astron. Astrophys., 327, 620-635, (1997). 3.2

[381] Soker, N., and Harpaz, A., "Criticism of recent calculations of common envelope ejection", Mon. Not. R. Astron. Soc., 343, 456-458, (2003). 3.5

[382] Solheim, J.-E., and Yungelson, L.R., "The White Dwarfs in AM CVn Systems - Candidates for SN Ia?", in Koester, D., and Moehler, S., eds., 14th European Workshop on White Dwarfs, Workshop in Kiel, Germany, 19 - 23 July 2004, vol. 334 of ASP Conference Series, 387, (Astronomical Society of the Pacific, San Francisco, U.S.A., 2005). Related online version (cited on 21 June 2006):

http://adsabs.harvard.edu/abs/2005ASPC. . 334 . .387S. 7

[383] Space Telescope Science Institute, "A Catalog and Atlas of Cataclysmic Variables", project homepage, (2006). URL (cited on 21 June 2006):

http://archive.stsci.edu/prepds/cvcat/index.html. 8

[384] Sparks, W.M., and Stecher, T.P., "Supernova: The Result of the Death Spiral of a White Dwarf into a Red Giant", Astrophys. J., 188, 149, (1974). Related online version (cited on 21 June 2006):

http://adsabs.harvard.edu/abs/1974ApJ...188_.149S. 3.5

[385] Spruit, H.C., and Phinney, E.S., "Birth kicks as the origin of pulsar rotation", Nature, 393, 139-141, (1998). Related online version (cited on 21 June 2006):

http://adsabs . harvard.edu/abs/1998Natur.393..139S. 3.4

[386] Stairs, I.H., "Testing General Relativity with Pulsar Timing", Living Rev. Relativity, 6, lrr2003-5, (2003). URL (cited on 15 Jan 2005):

http://www.livingreviews.org/lrr-2003-5. 2.1

[387] Stairs, I.H., Thorsett, S.E., and Arzoumanian, Z., "Measurement of Gravitational Spin-Orbit Coupling in a Binary-Pulsar System", Phys. Rev. Lett., 93, 141101, (2004). Related online version (cited on 21 June 2006):

http://adsabs. harvard.edu/abs/2004PhRvL. .93n1101S. 3.4

[388] Stairs, I.H., Thorsett, S.E., Taylor, J.H., and Wolszczan, A., "Studies of the Relativistic Binary Pulsar PSR B1534+12. I. Timing Analysis", Astrophys. J., 581, 501-508, (2002). 2.1

[389] Stanek, K.Z., Matheson, T., Garnavich, P.M., Martini, P., Berlind, P., Caldwell, N., Challis, P.and Brown, W.R., Schild, R., Krisciunas, K., Calkins, M.L., Lee, J.C., Hathi, N., Jansen, 
R.A., Windhorst, R.and Echevarria, L., Eisenstein, D.J., Pindor, B., Olszewski, E.W., Harding, P., Holland, S.T., and Bersier, D., "Spectroscopic Discovery of the Supernova 2003dh Associated with GRB 030329", Astrophys. J. Lett., 591, L17-L20, (2003). 2.3

[390] Stroeer, A., and Vecchio, A., "The LISA verification binaries", Class. Quantum Grav., 23, S809-S818, (2006). Related online version (cited on 21 June 2006):

http://arXiv.org/abs/astro-ph/0605227. 1, 8, 10

[391] Stroeer, A., Vecchio, A., and Nelemans, G., "LISA Astronomy of Double White Dwarf Binary Systems", Astrophys. J. Lett., 633, L33-L36, (2005). Related online version (cited on 21 June 2006):

http://adsabs .harvard.edu/abs/2005ApJ. . 633L. .33S. 12.2

[392] Sutantyo, W., "Asymmetric supernova explosions and the origin of binary pulsars", Astrophys. Space Sci., 54, 479-488, (1978). Related online version (cited on 4 November 2006): http://adsabs.harvard.edu/abs/1978Ap\&SS . 54 . 479S. 3.3

[393] Taam, R.E., and Sandquist, E.L., "Common Envelope Evolution of Massive Binary Stars", Annu. Rev. Astron. Astrophys., 38, 113-141, (2000). Related online version (cited on 4 November 2006):

http://adsabs.harvard.edu/abs/2000ARA\&A. .38. .113T. 3.5

[394] Taam, R.E., and van den Heuvel, E.P.J., "Magnetic field decay and the origin of neutron star binaries", Astrophys. J., 305, 235-245, (1986). Related online version (cited on 21 June 2006):

http://adsabs.harvard.edu/abs/1986ApJ...305. .235T. 7

[395] Taam, R.E., and Wade, R.A., "Angular momentum loss and the evolution of binaries of extreme mass ratio", Astrophys. J., 293, 504-507, (1985). Related online version (cited on 21 June 2006):

http://adsabs.harvard.edu/abs/1985ApJ...293. .504T. 9

[396] Takahashi, R., and Seto, N., "Parameter Estimation for Galactic Binaries by the Laser Interferometer Space Antenna", Astrophys. J., 575, 1030-1036, (2002). Related online version (cited on 21 June 2006):

http://adsabs.harvard.edu/abs/2002ApJ. ..575.1030T. 12.2

[397] Tauris, T.M., and Takens, R.J., "Runaway velocities of stellar components originating from disrupted binaries via asymmetric supernova explosions", Astron. Astrophys., 330, 10471059, (1998). URL (cited on 4 November 2006):

http://ads.inasan.rssi.ru/abs/1998A\&A. . 330.1047T. 3.3

[398] Tauris, T.M., and van den Heuvel, E.P.J., "New Direct Observational Evidence for Kicks in SNe", in Kramer, M., Wex, N., and Wielebinski, R., eds., Pulsar Astronomy: 2000 and Beyond (IAU Colloquium 177), 177th Colloquium of the IAU at the Max-Planck-Institut für Radioastronomie, Bonn, Germany, 30 August - 3 September 1999, ASP Conference Series, 595, (Astronomical Society of the Pacific, San Francisco, U.S.A., 2000). 3.4

[399] Thorne, K.S., "Gravitational radiation", in Hawking, S.W., and Israel, W., eds., Three Hundred Years of Gravitation, 330-458, (Cambridge University Press, Cambridge, U.K.; New York, U.S.A., 1987). 6.1

[400] Thorsett, S.E., and Chakrabarty, D., "Neutron Star Mass Measurements. I. Radio Pulsars", Astrophys. J., 512, 288-299, (1999). 1 
[401] Timmes, F.X., Diehl, R., and Hartmann, D.H., "Constraints from 26Al Measurements on the Galaxy's Recent Global Star Formation Rate and Core-Collapse Supernovae Rate", Astrophys. J., 479, 760, (1997). 2.2, 5.1

[402] Timmes, F.X., Woosley, S.E., and Taam, R.E., "The conductive propagation of nuclear flames. II. Convectively bounded flames in $\mathrm{C}+\mathrm{O}$ and $\mathrm{O}+\mathrm{NE}+\mathrm{MG}$ cores", Astrophys. J., 420, 348-363, (1994). Related online version (cited on 21 June 2006): http://adsabs.harvard.edu/abs/1994ApJ. . .420. 348T. 7

[403] Timmes, F.X., Woosley, S.E., and Weaver, T.A., "The Neutron Star and Black Hole Initial Mass Function", Astrophys. J., 457, 834-843, (1996). 4.2

[404] Tovmassian, G.H., Napiwotzki, R., Richer, M.G., Stasińska, G., Fullerton, A.W., and Rauch, T., "A Close Binary Nucleus in the MOST Oxygen-Poor Planetary Nebula PN G135.9+55.9", Astrophys. J., 616, 485-497, (2004). Related online version (cited on 21 June 2006): http: //adsabs.harvard.edu/abs/2004ApJ . .616 .485T. 8.1, 7

[405] Truran, J.W., and Livio, M., "On the frequency of occurrence of oxygen-neon-magnesium white dwarfs in classical nova systems", Astrophys. J., 308, 721-727, (1986). Related online version (cited on 21 June 2006): http://adsabs.harvard.edu/abs/1986ApJ...308. .721T. 1

[406] Tsugawa, M., and Osaki, Y., "Disk instability model for the AM Canum Venaticorum stars", Publ. Astron. Soc. Japan, 49, 75-84, (1997). Related online version (cited on 21 June 2006): http://adsabs.harvard.edu/abs/1997PASJ. . 49..75T. 9, 11, 18

[407] Turolla, R., Possenti, A., and Treves, A., "Is the bursting radio-source J1745-3009 a double neutron star binary?", Astrophys. J. Lett., 628, L49-L52, (2005). Related online version (cited on 21 June 2006): http://adsabs.harvard.edu/abs/2005ApJ . .628L . 49T. 2.2

[408] Tutukov, A., and Yungelson, L.R., "Evolution of close binary with relativistic component", Nauchn. Inform., 27, 86, (1973). Related online version (cited on 21 June 2006): http://adsabs.harvard.edu/abs/1973NInfo..27 . . 86T. In Russian. 4.1

[409] Tutukov, A.V., and Fedorova, A.V., "Formation and evolution of close binaries containing helium donors", Sov. Astron., 33, 606-613, (1989). 9

[410] Tutukov, A.V., and Fedorova, A.V., "Evolution of Close Stellar Binaries with Black Holes under the Action of Gravitational Radiation and Magnetic and Induced Stellar Winds of the Donor", Astron. Rep., 46, 765-778, (2002). Related online version (cited on 21 June 2006): http://adsabs.harvard.edu/abs/2002ARep...46. .765T. 4.1

[411] Tutukov, A.V., Fedorova, A.V., Ergma, E.V., and Yungelson, L.R., "Evolution of Low-Mass Close Binaries - The Minimum Orbital Period", Sov. Astron. Lett., 11, 52, (1985). Related online version (cited on 21 June 2006): http://adsabs.harvard.edu/abs/1985SvAL...11...52T. 7, 8, 9

[412] Tutukov, A.V., Fedorova, A.V., Ergma, E.V., and Yungelson, L.R., "The evolutionary status of MXB 1820-30 and other short-period low-mass X-ray sources", Sov. Astron. Lett., 13, 328, (1987). Related online version (cited on 21 June 2006): http://adsabs.harvard.edu/abs/1987SvAL...13..328T. 7, 9

[413] Tutukov, A.V., Fedorova, A.V., and Yungelson, L.R., "The evolution of dwarf binaries", Sov. Astron. Lett., 8, 365-370, (1982). 7 
[414] Tutukov, A.V., and Khokhlov, A.M., "Helium Shell Explosions in Accreting Carbon-Oxygen Dwarfs", Sov. Astron., 36, 401, (1992). Related online version (cited on 21 June 2006): http://adsabs.harvard.edu/abs/1992SvA....36. .401T. 7

[415] Tutukov, A.V., and Yungelson, L.R., "Evolution of massive close binaries", Nauchn. Inform., 27, 70, (1973). Related online version (cited on 21 June 2006): http://adsabs.harvard.edu/abs/1973NInfo..27...70T. 3.5, 4.1, 4.1

[416] Tutukov, A.V., and Yungelson, L.R., "Evolution of massive common envelope binaries and mass loss", in de Loore, C., and Conti, P.S., eds., Mass loss and evolution of O-type stars, IAU Symposium 83 in Vancouver Island, Canada, 5 - 9 June, 1978, 401, (D. Reidel, Dordrecht, Netherlands; Boston, U.S.A., 1979). 3.5

[417] Tutukov, A.V., and Yungelson, L.R., "On the influence of emission of gravitational waves on the evolution of low-mass close binary stars", Acta Astron., 29, 665-680, (1979). Related online version (cited on 21 June 2006):

http://adsabs.harvard.edu/abs/1979AcA...29.665T. 7, 7, 7, 8, 9, 10

[418] Tutukov, A.V., and Yungelson, L.R., "Evolutionary scenario for close binary systems of low and moderate masses", Nauchn. Inform., 49, 3, (1981). 7, 7, 8

[419] Tutukov, A.V., and Yungelson, L.R., "Formation of neutron stars in binary systems", Astron. Rep., 37, 411-431, (1993). 4.1, 5.1

[420] Tutukov, A.V., and Yungelson, L.R., "The merger rate of neutron star and black hole binaries", Mon. Not. R. Astron. Soc., 260, 675-678, (1993). 2.2, 4.1, 5.1, 5.2, 5.2, 6.1

[421] Tutukov, A.V., and Yungelson, L.R., "Merging of Binary White Dwarfs, Neutron Stars and Black-Holes Under the Influence of Gravitational Wave Radiation", Mon. Not. R. Astron. Soc., 268, 871, (1994). 7

[422] Tutukov, A.V., and Yungelson, L.R., "Double-degenerate semidetached binaries with helium secondaries: cataclismic variables, supersoft X-ray sources, supernovae and accretion-induced collapses", Mon. Not. R. Astron. Soc., 280, 1035-1045, (1996). 7, 9, 9, 12.1

[423] Tutukov, A.V., and Yungelson, L.R., "A Model for the Population of Binary Stars in the Galaxy", Astron. Rep., 46, 667-683, (2002). Related online version (cited on 21 June 2006): http://adsabs.harvard.edu/abs/2002ARep...46.667T. 5.2, 6, 7, 7, 10

[424] Ulla, A., "The X-ray properties of AM Canum Venaticorum", Astron. Astrophys., 301, 469, (1995). Related online version (cited on 21 June 2006): http://adsabs.harvard.edu/abs/1995A\&A...301..469U. 11

[425] van den Heuvel, E.P.J., "Formation and evolution of X-ray binaries", in Lewin, W.H.G., and van den Heuvel, E.P.J., eds., Accretion-Driven Stellar X-ray Sources, vol. 4 of Cambridge Astrophysics Series, 303-341, (Cambridge University Press, Cambridge, U.K.; New York, U.S.A., 1983). 3, 3.2.2

[426] van den Heuvel, E.P.J., Bhattacharya, D., Nomoto, K., and Rappaport, S.A., "Accreting white dwarf models for CAL 83, CAL 87 and other ultrasoft X-ray sources in the LMC", Astron. Astrophys., 262, 97-105, (1992). Related online version (cited on 21 June 2006): http: //adsabs.harvard.edu/abs/1992A\&A. .262..97V. 7

[427] van den Heuvel, E.P.J., and de Loore, C., "The nature of X-ray binaries. III. Evolution of massive close binarieswith one collapsed component, with a possible application to Cygnus X3", Astron. Astrophys., 25, 387, (1973). 4.1 
[428] van den Heuvel, E.P.J., and Habets, G.M.H.J., "Observational lower mass limit for black hole formation derived from massive X-ray binaries", Nature, 309, 598-600, (1984). 4.2

[429] van der Sluys, M.V., Verbunt, F., and Pols, O.R., "Creating ultra-compact binaries through stable mass transfer", in Burderi, L., Antonelli, L.A., D'Antona, F., di Salvo, T., Israel, G.L., Piersanti, L., Tornambè, A., and Straniero, O., eds., Interacting Binaries: Accretion, Evolution, and Outcomes, Conference in Cefalù, Italy, 4 - 10 July 2004, vol. 797 of AIP Conference Proceedings, 627-630, (American Institute of Physics, Melville, U.S.A., 2005). Related online version (cited on 21 June 2006):

http://adsabs.harvard.edu/abs/2005AIPC. .797. .627V. 7

[430] van der Sluys, M.V., Verbunt, F., and Pols, O.R., "Reduced magnetic braking and the magnetic capture model for the formation of ultra-compact binaries", Astron. Astrophys., 440, 973-979, (2005). Related online version (cited on 4 November 2006):

http://adsabs.harvard.edu/abs/2005A\&A...440..973V. 7

[431] van der Sluys, M.V., Verbunt, F., and Pols, O.R., "Modelling the formation of double white dwarfs", Astron. Astrophys., 460, 209-228, (2006). Related online version (cited on 4 November 2006):

http://adsabs.harvard.edu/abs/2006A\&A. . 460. . 209V. 3.5

[432] Verbunt, F., "X-ray sources in globular clusters", in Burderi, L., Antonelli, L.A., D'Antona, F., di Salvo, T., Israel, G.L., Piersanti, L., Tornambè, A., and Straniero, O., eds., Interacting Binaries: Accretion, Evolution, and Outcomes, Conference in Cefalù, Italy, 4 - 10 July 2004, vol. 797 of AIP Conference Proceedings, 30-39, (American Institute of Physics, Melville, U.S.A., 2005). Related online version (cited on 21 June 2006): http://adsabs.harvard.edu/abs/2005AIPC. .797 ...30V. 7

[433] Verbunt, F., and Rappaport, S., "Mass transfer instabilities due to angular momentum flows in close binaries", Astrophys. J., 332, 193-198, (1988). 9

[434] Verbunt, F., Wijers, R.A.M.J., and Burm, H.M.G., "Evolutionary scenarios for the X-ray binary pulsars $4 \mathrm{U} 1626-67$ and Hercules X-1, and their implications for the decay of neutron star magnetic fields", Astron. Astrophys., 234, 195-202, (1990). Related online version (cited on 21 June 2006):

http://adsabs.harvard.edu/abs/1990A\&A...234..195V. 7

[435] Verbunt, F., and Zwaan, C., "Magnetic braking in low-mass X-ray binaries", Astron. Astrophys., 100, L7-L9, (1981). Related online version (cited on 21 June 2006):

http://adsabs.harvard.edu/abs/1981A\&A...100L ...7V. 3.1.5, 8

[436] Vila, S.C., "Late Evolution of Close Binaries", Astrophys. J., 168, 217-223, (1971). Related online version (cited on 21 June 2006):

http://adsabs.harvard.edu/abs/1971ApJ...168. .217V. 9

[437] Voss, R., and Tauris, T.M., "Galactic distribution of merging neutron stars and black holes Prospects for short gamma-ray burst progenitors and LIGO/VIRGO", Mon. Not. R. Astron. Soc., 342, 1169-1184, (2003). Related online version (cited on 21 June 2006): http://adsabs.harvard.edu/abs/2003MNRAS.342.1169V. 5.2, 5.2

[438] Wade, R.A., "A double grid of accretion disc model spectra for cataclysmic variable stars", Mon. Not. R. Astron. Soc., 208, 381-398, (1984). Related online version (cited on 21 June 2006):

http://adsabs.harvard.edu/abs/1984MNRAS.208. .381W. 11 
[439] Wagner, R.M., Foltz, C.B., and Starrfield, S.G., "Possible Nova in Puppis", IAU Circ., 2001(7556), (January, 2001). Related online version (cited on 21 June 2006):

http://cfa-www.harvard.edu/iauc/07500/07556.html. 7

[440] Wagner, R.M., Schwarz, G., Starrfield, S.G., Foltz, C.B., Howell, S., and Szkody, P., "V445 Puppis", IAU Circ., 2001(7717), (September, 2001). Related online version (cited on 21 June 2006):

http://cfa-www.harvard.edu/iauc/07700/07717.html. 7

[441] Wang, Q.D., Gotthelf, E.V., and Lang, C.C., "A faint discrete source origin for the highly ionized iron emission from the Galactic Centre region", Nature, 415, 148-150, (2002). Related online version (cited on 21 June 2006):

http://adsabs.harvard.edu/abs/2002Natur.415..148W. 12

[442] Warner, B., and Robinson, E.L., "Observations of rapid blue variables - IX. AM CVn (HZ 29)", Mon. Not. R. Astron. Soc., 159, 101-111, (1972). Related online version (cited on 21 June 2006):

http://adsabs.harvard.edu/abs/1972MNRAS.159..101W. 7

[443] Webbink, R.F., "The formation of the white dwarfs in close binary systems", in van Horn, H.M., and Weidemann, V., eds., White Dwarfs and Variable Degenerate Stars (IAU Colloquium 53), Colloquium, July 30 - August 21979 and Fourth Annual Workshop on Novae, Dwarf Novae and Other Cataclysmic Variables at the University of Rochester in Rochester, N.Y., 3 August 1979, 426-447, (University of Rochester, Rochester, U.S.A., 1979). 7, 7, 8

[444] Webbink, R.F., "Double white dwarfs as progenitors of R Coronae Borealis stars and Type I supernovae", Astrophys. J., 277, 355-360, (1984). 3.5, 7, 8

[445] Webbink, R.F., and Han, Z., "Gravitational Radiation from Close Double White Dwarfs", in Folkner, W.M., ed., Laser Interferometer Space Antenna, Second International LISA Symposium on the Detection and Observation of Gravitational Waves in Space, Pasadena, California, July 1998, vol. 456 of AIP Conference Proceedings, 61, (American Institure of Physics, Woodbury, U.S.A., 1998). Related online version (cited on 21 June 2006):

http://adsabs.harvard.edu/abs/1998lain.conf...61W. 10, 10

[446] Webbink, R.F., and Iben Jr, I., "Tidal interaction and coalescence of close binary white dwarfs", in Philip, A.G.D., Hayes, D.S., and Liebert, J.W., eds., The Second Conference on Faint Blue Stars (IAU Colloquium 95), Conference in Tucson, Arizona, 1 - 5 June 1987, 445-456, (L. Davis Press, Schenectady, U.S.A., 1987). Related online version (cited on 4 November 2006):

http://adsabs.harvard.edu/abs/1987fbs..conf. .445W. 9

[447] Weisberg, J.M., and Taylor, J.H., "General Relativistic Geodetic Spin Precession in Binary Pulsar B1913+16: Mapping the Emission Beam in Two Dimensions", Astrophys. J., 576, 942-949, (2002). 3.4

[448] Werner, K., Nagel, T., Rauch, T., Hammer, N.J., and Dreizler, S., "VLT spectroscopy and non-LTE modeling of the $\mathrm{C} / \mathrm{O}$-dominated accretion disks in two ultracompact X-ray binaries", Astron. Astrophys., 450, 725-733, (2006). Related online version (cited on 21 June 2006):

http://adsabs.harvard.edu/abs/2006A\&A...450..725W. 7

[449] Wettig, T., and Brown, G.E., "The evolution of relativistic binary pulsars", New Astronomy, 1, 17-34, (1996). 4.1 
[450] Wex, N., Kalogera, V., and Kramer, M., "Constraints on Supernova Kicks from the Double Neutron Star System PSR B1913+16", Astrophys. J., 528, 401-409, (2000). 3.4

[451] Whelan, J., and Iben Jr, I., "Binaries and Supernovae of Type I", Astrophys. J., 186, 10071014, (1973). Related online version (cited on 21 June 2006): http://adsabs.harvard.edu/abs/1973ApJ ...186.1007W. 7

[452] Willems, B., Henninger, M., Levin, T., Ivanova, N., Kalogera, V., McGhee, K., Timmes, F.X., and Fryer, C.L., "Understanding Compact Object Formation and Natal Kicks. I. Calculation Methods and the Case of GRO J1655-40", Astrophys. J., 625, 324-346, (2005). 3.4

[453] Willems, B., and Kalogera, V., "Constraints on the Formation of PSR J0737-3039: The Most Probable Isotropic Kick Magnitude", Astrophys. J. Lett., 603, L101-L104, (2004). 3.4, 4.1

[454] Willems, B., Kalogera, V., and Henninger, M., "Pulsar Kicks and Spin Tilts in the Close Double Neutron Stars PSR J0737-3039, PSR B1534+12, and PSR B1913+16", Astrophys. J., 616, 414-438, (2004). 3.4

[455] Wolszczan, A., "PSR 1257+12 and PSR 1534+12", IAU Circ., 1990(5073), (August, 1990). Related online version (cited on 13 November 2006): http://cfa-www.harvard.edu/iauc/05000/05073.html. 2.1

[456] Woosley, S.E., and Heger, A., "The Progenitor Stars of Gamma-Ray Bursts", Astrophys. J., 637, 914-921, (2006). Related online version (cited on 21 June 2006): http://adsabs.harvard.edu/abs/2006ApJ ..637..914W. 1

[457] Woosley, S.E., Heger, A., and Weaver, T.A., "The evolution and explosion of massive stars", Rev. Mod. Phys., 74, 1015-1071, (2002). 1

[458] Woosley, S.E., Langer, N., and Weaver, T.A., "The Presupernova Evolution and Explosion of Helium Stars That Experience Mass Loss", Astrophys. J., 448, 315, (1995). 4.2

[459] Woosley, S.E., and Weaver, T.A., "Sub-Chandrasekhar mass models for Type Ia supernovae", Astrophys. J., 423, 371-379, (1994). Related online version (cited on 21 June 2006): http://adsabs. harvard.edu/abs/1994ApJ . .423..371W. 7

[460] Yamaoka, H., Shigeyama, T., and Nomoto, K., "Formation of double neutron star systems and asymmetric supernova explosions", Astron. Astrophys., 267, 433-438, (1993). 3.3

[461] Yaron, O., Prialnik, D., Shara, M.M., and Kovetz, A., "An Extended Grid of Nova Models. II. The Parameter Space of Nova Outbursts", Astrophys. J., 623, 398-410, (2005). Related online version (cited on 21 June 2006): http://adsabs.harvard.edu/abs/2005ApJ . .623. .398Y. 7

[462] Yoon, S.-C., and Langer, N., "The first binary star evolution model producing a Chandrasekhar mass white dwarf", Astron. Astrophys., 412, L53-L56, (2003). Related online version (cited on 21 June 2006):

http://adsabs.harvard.edu/abs/2003A\&A...412L. .53Y. 7

[463] Yoon, S.-C., and Langer, N., "Helium accreting CO white dwarfs with rotation: Helium novae instead of double detonation", Astron. Astrophys., 419, 645-652, (2004). 7

[464] Yoon, S.-C., and Langer, N., "On the evolution of rapidly rotating massive white dwarfs towards supernovae or collapses", Astron. Astrophys., 435, 967-985, (2005). Related online version (cited on 4 November 2006):

http://adsabs.harvard.edu/abs/2005A\&A...435..967Y. 7 
[465] Yoon, S.-C., Langer, N., and van der Sluys, M.V., "On the stability of thermonuclear shell sources in stars", Astron. Astrophys., 425, 207-216, (2004). Related online version (cited on 4 November 2006):

http://adsabs.harvard.edu/abs/2004A\&A..425..207Y. 7

[466] Yungelson, L.R., "Evolution of close binaries with mass loss from the system. III. Systems containing white dwarfs", Nauchn. Inform., 26, 71-82, (1973). Related online version (cited on 4 November 2006):

http://adsabs.harvard.edu/abs/1973NInfo..26...71Y. In Russian. 3.2.3

[467] Yungelson, L.R., "Population synthesis for low and intermediate mass binaries", in Burderi, L., Antonelli, L.A., D’Antona, F., di Salvo, T., Israel, G.L., Piersanti, L., Tornambè, A., and Straniero, O., eds., Interacting Binaries: Accretion, Evolution, and Outcomes, Conference in Cefalù, Italy, 4 - 10 July 2004, vol. 797 of AIP Conference Proceedings, 1-10, (American Institute of Physics, Melville, U.S.A., 2005). Related online version (cited on 21 June 2006): http://adsabs.harvard.edu/abs/2005AIPC. .797...1Y. 5.2

[468] Yungelson, L.R., "Population Synthesis for Progenitors of Type Ia Supernovae", in Sion, E.M., Vennes, S., and H.L., Shipman, eds., White Dwarfs: Galactic and Cosmological Probes, Astrophysics and Space Science Library, (Springer, Dordrecht, Netherlands, 2005). Related online version (cited on 4 November 2006):

http://arXiv.org/abs/astro-ph/0409677. 5

[469] Yungelson, L.R., Lasota, J.-P., Nelemans, G., Dubus, G., van den Heuvel, E.P.J., Dewi, J., and Portegies Zwart, S., "The origin and fate of short-period low-mass black-hole binaries", Astron. Astrophys., 454, 559-569, (2006). Related online version (cited on 21 June 2006): http://adsabs.harvard.edu/abs/2006A\&A...454..559Y. 3.4, 5.2

[470] Yungelson, L.R., and Livio, M., "Type Ia Supernovae: An Examination of Potential Progenitors and the Redshift Distribution", Astrophys. J., 497, 168, (1998). 1, 7, 7, 7

[471] Yungelson, L.R., Livio, M., Truran, J.W., Tutukov, A., and Fedorova, A., "A Model for the Galactic Population of Binary Supersoft X-Ray Sources", Astrophys. J., 466, 890, (1996). 7

[472] Yungelson, L.R., Nelemans, G., and van den Heuvel, E.P.J., "On the formation of neonenriched donor stars in ultracompact X-ray binaries", Astron. Astrophys., 388, 546-551, (2002). 7, 9, 10

[473] Yungelson, L.R., and Tutukov, A.V., "A Model for the Population of Helium Stars in the Galaxy: Low-Mass Stars", Astron. Rep., 49, 871-883, (2005). Related online version (cited on 21 June 2006):

http://adsabs.harvard.edu/abs/2005ARep...49..871Y. 7

[474] Yungelson, L.R., Tutukov, A.V., and Livio, M., "The formation of binary and single nuclei of Planetary Nebulae", Astrophys. J., 418, 794-803, (1993). 3.5

[475] Zel'dovich, Y.B., Ivanova, L.N., and Nadezhin, D.K., "Nonstationary Hydrodynamical Accretion onto a Neutron Star", Sov. Astron., 16, 209, (1972). Related online version (cited on 4 November 2006):

http://adsabs.harvard.edu/abs/1972SvA...16..209Z. 6 